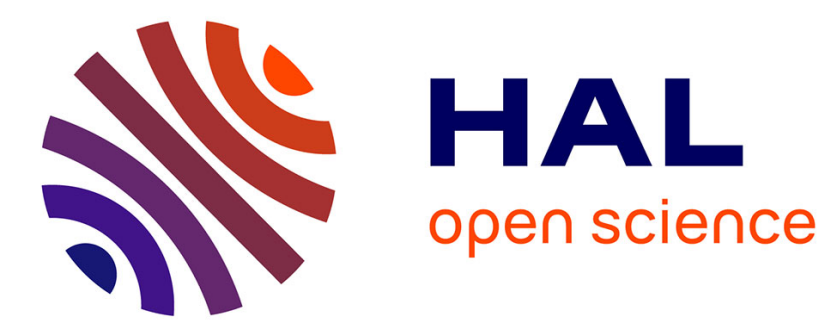

\title{
Traveling waves for the Nonlinear Schrödinger Equation with general nonlinearity in dimension one
}

David Chiron

\section{To cite this version:}

David Chiron. Traveling waves for the Nonlinear Schrödinger Equation with general nonlinearity in dimension one. Nonlinearity, 2012, 25 (3), pp.813-850. 10.1088/0951-7715/25/3/813 . hal-00809123

\section{HAL Id: hal-00809123 \\ https://hal.science/hal-00809123}

Submitted on 8 Apr 2013

HAL is a multi-disciplinary open access archive for the deposit and dissemination of scientific research documents, whether they are published or not. The documents may come from teaching and research institutions in France or abroad, or from public or private research centers.
L'archive ouverte pluridisciplinaire HAL, est destinée au dépôt et à la diffusion de documents scientifiques de niveau recherche, publiés ou non, émanant des établissements d'enseignement et de recherche français ou étrangers, des laboratoires publics ou privés. 


\title{
Traveling waves for the Nonlinear Schrödinger Equation with general nonlinearity in dimension one
}

\author{
D. Chiron*
}

\begin{abstract}
We study the traveling waves of the Nonlinear Schrödinger Equation in dimension one. Through various model cases, we show that for nonlinearities having the same qualitative behaviour as the standard Gross-Pitaevkii one, the traveling waves may have rather different properties. In particular, our examples exhibit multiplicity or nonexistence results, cusps (as for the Jones-Roberts curve in the three-dimensional Gross-Pitaevskii equation), and a transonic limit which can be the modified (KdV) solitons or even the generalized $(\mathrm{KdV})$ soliton instead of the standard $(\mathrm{KdV})$ soliton.
\end{abstract}

Key-words: traveling wave, Nonlinear Schrödinger Equation, Gross-Pitaevskii Equation, Korteweg-de Vries soliton, (mKdV) solitons, (gKdV) soliton.

MSC (2010): 34B40, 34C99, 35B35, 35Q55.

\section{Introduction}

In this paper, we consider the Nonlinear Schrödinger Equation in dimension one

$$
i \frac{\partial \Psi}{\partial t}+\partial_{x}^{2} \Psi+\Psi f\left(|\Psi|^{2}\right)=0 .
$$

This equation appears as a relevant model in condensed matter physics: Bose-Einstein condensation and superfluidity (see [28], [16], [18], [1]); Nonlinear Optics (see, for instance, the survey [22]). The nonlinearity $f$ may be $f(\varrho)= \pm \varrho$ or $f(\varrho)=1-\varrho$, in which case (NLS) is termed the Gross-Pitaevskii equation, or $f(\varrho)=-\varrho^{2}$ (see, e.g., [23]) in the context of Bose-Einstein condensates, and more generally a pure power. The so-called "cubic-quintic" (NLS), where, for some positive constants $\alpha_{1}, \alpha_{3}$ and $\alpha_{5}$,

$$
f(\varrho)=-\alpha_{1}+\alpha_{3} \varrho-\alpha_{5} \varrho^{2}
$$

and $f$ has two positive roots, is also of high interest in physics (see, e.g., [5]). We shall focus on the one dimensional case, which appears quite often in Nonlinear Optics. In this context, the nonlinearity can take various forms (see [22]):

$$
f(\varrho)=-\alpha \varrho^{\nu}-\beta \varrho^{2 \nu}, \quad f(\varrho)=-\frac{\varrho_{0}}{2}\left(\frac{1}{\left(1+\frac{1}{\varrho_{0}}\right)^{\nu}}-\frac{1}{\left(1+\frac{\varrho}{\varrho_{0}}\right)^{\nu}}\right), \quad f(\varrho)=-\alpha \varrho\left(1+\gamma \tanh \left(\frac{\varrho^{2}-\varrho_{0}^{2}}{\sigma^{2}}\right)\right) \ldots
$$

where $\alpha, \beta, \gamma, \nu, \sigma$ are given constants (the second one, for instance, takes into account saturation effects). For the first nonlinearity in (1), we usually have $\alpha \beta<0$, hence it is close, in some sense, to the cubicquintic nonlinearity. Therefore, it is natural to allow the nonlinearity to be quite general. In the context of Bose-Einstein condensation or Nonlinear Optics, the natural condition at infinity appears to be

$$
|\Psi|^{2} \rightarrow r_{0}^{2} \quad \text { as } \quad|x| \rightarrow+\infty,
$$

*Laboratoire J.A. Dieudonné, Université de Nice-Sophia Antipolis, Parc Valrose, 06108 Nice Cedex 02, France. e-mail: chiron@unice.fr. 
where $r_{0}>0$ is such that $f\left(r_{0}^{2}\right)=0$. We shall assume throughout the paper $f$ as smooth as required.

For solutions $\Psi$ of (NLS) which do not vanish, we may use the Madelung transform

$$
\Psi=a \exp (i \phi)
$$

and rewrite (NLS) as an hydrodynamical system with an additional quantum pressure

$$
\left\{\begin{array} { l } 
{ \partial _ { t } a + 2 \partial _ { x } \phi \partial _ { x } a + a \partial _ { x } ^ { 2 } \phi = 0 } \\
{ \partial _ { t } \phi + ( \partial _ { x } \phi ) ^ { 2 } - f ( a ^ { 2 } ) - \frac { \partial _ { x } ^ { 2 } a } { a } = 0 }
\end{array} \quad \text { or } \quad \left\{\begin{array}{l}
\partial_{t} \rho+2 \partial_{x}(\rho u)=0 \\
\partial_{t} u+2 u \partial_{x} u-\partial_{x}(f(\rho))-\partial_{x}\left(\frac{\partial_{x}^{2}(\sqrt{\rho})}{\sqrt{\rho}}\right)=0
\end{array}\right.\right.
$$

with $(\rho, u) \equiv\left(a^{2}, \partial_{x} \phi\right)$. When neglecting the quantum pressure and linearizing this Euler system around the particular trivial solution $\Psi=r_{0}\left(\right.$ or $\left.(a, u)=\left(r_{0}, 0\right)\right)$, we obtain the free wave equation

$$
\left\{\begin{array}{l}
\partial_{t} \bar{a}+r_{0} \partial_{x} \bar{u}=0 \\
\partial_{t} \bar{u}-2 r_{0} f^{\prime}\left(r_{0}^{2}\right) \partial_{x} \bar{a}=0
\end{array}\right.
$$

with associated speed of sound

$$
\mathfrak{c}_{s} \equiv \sqrt{-2 r_{0}^{2} f^{\prime}\left(r_{0}^{2}\right)}>0
$$

provided $f^{\prime}\left(r_{0}^{2}\right)<0$ (that is the Euler system is hyperbolic in the region $\rho \simeq r_{0}^{2}$ ), which we will assume throughout the paper. The speed of sound plays a crucial role in the existence of traveling waves for (NLS).

The Nonlinear Schrödinger equation formally preserves the energy

$$
E(\Psi) \equiv \int_{\mathbb{R}}\left|\partial_{x} \Psi\right|^{2}+V\left(|\Psi|^{2}\right) d x
$$

where $V(\varrho)=\int_{\varrho}^{r_{0}^{2}} f(s) d s$, as well as the momentum. The momentum is not easy to define in dimension one for maps that vanish somewhere (see [6], [7]). However, if $\Psi$ does not vanish, we may lift $\Psi=a \mathrm{e}^{i \phi}$, and then the momentum is defined (see [8]) by

$$
P(\Psi) \equiv \int_{\mathbb{R}}\left\langle i \Psi, \partial_{x} \Psi\right\rangle\left(1-\frac{r_{0}^{2}}{|\Psi|^{2}}\right) d x=\int_{\mathbb{R}}\left(a^{2}-r_{0}^{2}\right) \partial_{x} \phi d x,
$$

where $\langle\cdot, \cdot\rangle$ denotes the real scalar product in $\mathbb{C}$.

\subsection{The traveling waves}

The traveling waves play an important role in the long time dynamics of (NLS) with nonzero condition at infinity. These are solutions of (NLS) of the form

$$
\Psi(t, x)=U(x-c t)
$$

where $c$ is the speed of propagation. The profile $U$ has to solve the ODE

$$
\partial_{x}^{2} U+U f\left(|U|^{2}\right)=i c \partial_{x} U
$$

together with the condition $|U(x)| \rightarrow r_{0}$ as $x \rightarrow \pm \infty$. We may without loss of generality assume that $c \geq 0$ (otherwise we consider the complex conjugate $\bar{U}$ instead of $U$ ). Moreover, we shall restrict ourselves to finite energy traveling waves, in the sense that $\partial_{x} U \in L^{2}(\mathbb{R})$ and $|U|^{2}-r_{0}^{2} \in L^{2}(\mathbb{R})$. In what follows, (nontrivial) traveling wave then means a (nontrivial) solution to $\left(\mathrm{TW}_{c}\right)$ with $|U(x)| \rightarrow r_{0}$ as $x \rightarrow \pm \infty$ and finite energy. Let $U$ be such a traveling wave. Taking the scalar product of $\left(\mathrm{TW}_{c}\right)$ with $2 \partial_{x} U$, we deduce

$$
\partial_{x}\left(\left|\partial_{x} U\right|^{2}-V\left(|U|^{2}\right)\right)=0 \quad \text { in } \mathbb{R},
$$


hence

$$
\left|\partial_{x} U\right|^{2}=V\left(|U|^{2}\right) \quad \text { in } \mathbb{R},
$$

in view of the condition at infinity and since $U$ has finite energy. Similarly, denoting $U=U_{1}+i U_{2}$ and taking the scalar product of $\left(\mathrm{TW}_{c}\right)$ with $i U$ and $U$ respectively yields, for some constant $K$,

$$
U_{1} \partial_{x} U_{2}-U_{2} \partial_{x} U_{1}=\frac{c}{2}\left(|U|^{2}-r_{0}^{2}\right)+K \quad \text { in } \mathbb{R}
$$

after integration and

$$
\left\langle U, \partial_{x}^{2} U\right\rangle+|U|^{2} f\left(|U|^{2}\right)=-c\left(U_{1} \partial_{x} U_{2}-U_{2} \partial_{x} U_{1}\right) \quad \text { in } \mathbb{R} .
$$

Equation (4) allows one to compute the phase of $U$ when $U$ does not vanish. Indeed, on each interval where $U$ does not vanish one may write $U=a \mathrm{e}^{i \phi}$ and (4) becomes

$$
a^{2} \partial_{x} \phi=\frac{c}{2} \eta+K
$$

Since we restrict ourselves to traveling waves with finite energy, we must have $K=0$ in (4). Indeed, $|U| \rightarrow r_{0}>0$ as $|x| \rightarrow+\infty$, hence $U$ has a lifting $U=a \mathrm{e}^{i \phi}$ with $a \geq r_{0} / 2$ for large $|x|$, say $|x| \geq R$, and since $\int_{\{|x| \geq R\}}\left(\partial_{x} \phi\right)^{2}+\eta^{2} d x \leq \int_{\{|x| \geq R\}}\left(2 / r_{0}\right)^{2}\left|\partial_{x} U\right|^{2}+\left(|U|^{2}-r_{0}^{2}\right)^{2} d x<\infty$ by assumption, this imposes $K=0$. Therefore, combining (3), (4) (with $K=0$ ) and using the identity $\partial_{x}^{2}\left(|U|^{2}\right)=2\left\langle U, \partial_{x}^{2} U\right\rangle+2\left|\partial_{x} U\right|^{2}$, we infer that the function

$$
\eta \equiv|U|^{2}-r_{0}^{2}
$$

solves the ODE

$$
\partial_{x}^{2} \eta+c^{2} \eta-2 V\left(r_{0}^{2}+\eta\right)+2\left(r_{0}^{2}+\eta\right) f\left(r_{0}^{2}+\eta\right)=0 .
$$

This last equation can be written under the form of a Newton type equation

$$
\partial_{x}^{2} \eta+\frac{1}{2} \mathcal{V}^{\prime}(\eta)-\left(\mathfrak{c}_{s}^{2}-c^{2}\right) \eta=\partial_{x}^{2} \eta+\frac{1}{2} \frac{d}{d \eta}\left[\mathcal{V}(\eta)-\left(\mathfrak{c}_{s}^{2}-c^{2}\right) \eta^{2}\right]=0
$$

involving the potential function

$$
\left[-r_{0}^{2},+\infty\right) \ni \xi \mapsto \mathcal{V}_{c}(\xi) \equiv \mathcal{V}(\xi)-\varepsilon^{2} \xi^{2}=\mathcal{V}(\xi)-\left(\mathfrak{c}_{s}^{2}-c^{2}\right) \xi^{2},
$$

where the function $\mathcal{V}:\left[-r_{0}^{2},+\infty\right) \rightarrow \mathbb{R}$ is defined (see, e.g. [25], proof of Theorem 5.1) by

$$
\mathcal{V}(\xi) \equiv \mathfrak{c}_{s}^{2} \xi^{2}-4\left(r_{0}^{2}+\xi\right) V\left(r_{0}^{2}+\xi\right) .
$$

This type of differential equation is very classical (see, e.g. [2]) and is associated to the first integral

$$
\left(\partial_{x} \eta\right)^{2}+\mathcal{V}(\eta)-\left(\mathfrak{c}_{s}^{2}-c^{2}\right) \eta^{2}=0
$$

since $\eta \rightarrow 0$ at $\pm \infty$. By drawing the potential

$$
\left[-r_{0}^{2},+\infty\right) \ni \xi \mapsto \mathcal{V}_{c}(\xi)=\mathcal{V}(\xi)-\left(\mathfrak{c}_{s}^{2}-c^{2}\right) \xi^{2}
$$

in (9), it is easy to see if there exists or not a solution $\eta$ to (8) such that $\eta \rightarrow 0$ at infinity (that is, $|U| \rightarrow r_{0}$ at infinity). Indeed, a nontrivial solution $\eta$ to (7) with $\eta \rightarrow 0$ at infinity exists if and only if $\mathcal{V}_{c}$ is negative on some interval $\left(\xi_{*}, 0\right)$ or $\left(0, \xi_{*}\right)$, with $\xi_{*} \geq-r_{0}^{2}, \mathcal{V}_{c}\left(\xi_{*}\right)=0$ and $\mathcal{V}_{c}^{\prime}\left(\xi_{*}\right)>0$ (if $\xi_{*}>0$ ) or $\mathcal{V}_{c}^{\prime}\left(\xi_{*}\right)<0$ (if $-r_{0}^{2} \leq \xi_{*}<0$ ). Moreover, it is easy to see that $\eta$ is symmetric with respect to some point $x_{0}$ (at which $\eta$ is maximum $\left(\xi_{*}>0\right)$ or minimum $\left(\xi_{*}<0\right)$ ); we freeze the invariance by translation by imposing $x_{0}=0$ (that is, we require $|U|$ to be even).

In order to compute $U$ completely, we need to express the phase $\phi$. Assume first $c>0$. Then, we have

$$
\mathcal{V}_{c}(\xi)=\mathcal{V}(\xi)-\left(\mathfrak{c}_{s}^{2}-c^{2}\right) \xi^{2}=c^{2} \xi^{2}-4\left(r_{0}^{2}+\xi\right) V\left(r_{0}^{2}+\xi\right),
$$

and thus $\mathcal{V}_{c}\left(-r_{0}^{2}\right)=c^{2} r_{0}^{4}>0$. If $U$ is a traveling wave of speed $c>0$, we then see from the Newton equation (9) that we cannot have $\eta=-r_{0}^{2}$ at some point $x$, hence $U$ cannot vanish. Therefore, we may write $U=a \mathrm{e}^{i \phi}$ 
and (6) holds on $\mathbb{R}$. Consequently, the momentum of $U$ is well-defined. When $c=0$, the picture is slightly different. If $\xi_{*}>-r_{0}^{2}$, then we easily see that $|U|$ is even and does not vanish, hence (6) (with $c=0$ ) implies $\phi=C t e$ in $\mathbb{R}$, and such a solution is called a "bubble"; if $\xi_{*}=-r_{0}^{2}$, then we can construct by reflection an odd traveling wave solution $U$, called a "kink" (the phase $\phi(x)=\pi \mathbf{1}_{\mathbb{R}_{-}^{*}}(x)$ is then singular at the origin). Since the kink vanishes at the origin, its momentum can not be defined by the formula we have given.

An immediate consequence of the fact that $\eta$ obeys the Newton type equation (7) is the following result, essentially proved in [25], for non existence of sonic or supersonic nontrivial traveling waves.

Theorem 1 ([25]) Let $f \in \mathcal{C}^{2}\left(\mathbb{R}_{+}, \mathbb{R}\right)$ be such that $f^{\prime}\left(r_{0}^{2}\right)<0$.

a) If $c>\mathfrak{c}_{s}$, then there does not exist any nonconstant solution $U$ of $\left(\mathrm{TW}_{c}\right)$ such that $|U| \rightarrow r_{0}$ at $\pm \infty$.

b) A necessary and sufficient condition for the existence of a nonconstant solution $U$ of $\left(\mathrm{TW}_{\boldsymbol{c}_{s}}\right)$ satisfying $|U| \rightarrow r_{0}$ at $\pm \infty$ is that there exists $\xi_{*}>0$ (resp. $\left.-r_{0}^{2} \leq \xi_{*}<0\right)$ such that $\mathcal{V}<0$ in $\left(0, \xi_{*}\right)$ and $\mathcal{V}\left(\xi_{*}\right)=0<$ $\mathcal{V}^{\prime}\left(\xi_{*}\right)$ (resp. $\mathcal{V}<0$ in $\left(\xi_{*}, 0\right)$ and $\mathcal{V}\left(\xi_{*}\right)=0>\mathcal{V}^{\prime}\left(\xi_{*}\right)$ ).

Proof. For sake of completeness, we recall the proof of [25], which follows immediately from the behaviour of the function $\mathcal{V}_{c}$ at the origin. Indeed, note first that by definition of $\mathfrak{c}_{s}=\sqrt{-2 r_{0}^{2} f^{\prime}\left(r_{0}^{2}\right)}$, we have by Taylor expansion as $\xi \rightarrow 0$

$$
\mathcal{V}(\xi)=\mathfrak{c}_{s}^{2} \xi^{2}-4\left(r_{0}^{2}+\xi\right) V\left(r_{0}^{2}+\xi\right)=\mathfrak{c}_{s}^{2} \xi^{2}-4\left(r_{0}^{2}+\xi\right)\left(-\frac{1}{2} f^{\prime}\left(r_{0}^{2}\right) \xi^{2}+\mathcal{O}\left(\xi^{3}\right)\right)=\mathcal{O}\left(\xi^{3}\right),
$$

hence

$$
\mathcal{V}_{c}(\xi)=\mathcal{V}(\xi)-\left(\mathfrak{c}_{s}^{2}-c^{2}\right) \xi^{2}=\left(c^{2}-\mathfrak{c}_{s}^{2}\right) \xi^{2}+\mathcal{O}\left(\xi^{3}\right) .
$$

If $c>\mathfrak{c}_{s}$, then it follows that $\mathcal{V}_{c}(\xi)>0$ for $\xi$ small (depending on $c$ ), $\xi \neq 0$. Therefore, there can not exist non trivial traveling wave with finite energy if $c>\mathfrak{c}_{s}$. When $c=\mathfrak{c}_{s}, \mathcal{V}_{c}=\mathcal{V}$ and thus $b$ ) is the existence criterion for an arbitrary $c$.

Consequently, nontrivial traveling waves of finite energy do not exist outside the interval of speed $c \in$ $\left[0, \mathfrak{c}_{s}\right]$.

\subsection{Computation of energy and momentum}

Since a traveling wave $U$ of speed $0<c<\mathfrak{c}_{s}$ (and of finite energy) does not vanish, we may lift $U=a \mathrm{e}^{i \phi}$ and we have the equations

$$
a^{2} \partial_{x} \phi=\frac{c}{2} \eta \quad \text { and } \quad\left(\partial_{x} \eta\right)^{2}=\varepsilon^{2} \eta^{2}-\mathcal{V}(\eta)=-\mathcal{V}_{c}(\eta)
$$

Recall that we have imposed $|U|$ (or $\eta$ ) to be even, hence it is standard to show (see, e.g. [2]) that if $\xi_{c} \neq 0$ is a simple zero of $\mathcal{V}_{c}$ (positive or negative) such that $\mathcal{V}_{c}$ is negative between 0 and $\xi_{c}$, then

$$
\partial_{x} \eta=-\operatorname{sgn}\left(\xi_{c}\right) \sqrt{-\mathcal{V}_{c}(\eta)} \quad \text { in } \quad \mathbb{R}_{+} .
$$

Therefore, using the fact that $\eta$ is even and the change of variable $\xi=\eta(x)$ in $\mathbb{R}_{+}$, we get

$$
P(U)=\int_{\mathbb{R}}\left(a^{2}(x)-r_{0}^{2}\right) \partial_{x} \phi(x) d x=c \int_{0}^{+\infty} \frac{\eta^{2}(x)}{r_{0}^{2}+\eta(x)} d x=\operatorname{sgn}\left(\xi_{c}\right) c \int_{0}^{\xi_{c}} \frac{\xi^{2}}{r_{0}^{2}+\xi} \frac{d \xi}{\sqrt{\varepsilon^{2} \xi^{2}-\mathcal{V}(\xi)}},
$$

where $\xi_{c}$ is the zero of $\xi \mapsto \varepsilon^{2} \xi^{2}-\mathcal{V}(\xi)=-\mathcal{V}_{c}(\xi)$ of interest. For the energy, we may first use (3) to infer

$$
E(U)=2 \int_{\mathbb{R}} V\left(a^{2}\right) d x=4 \operatorname{sgn}\left(\xi_{c}\right) \int_{0}^{\xi_{c}} \frac{V\left(r_{0}^{2}+\xi\right)}{\sqrt{\varepsilon^{2} \xi^{2}-\mathcal{V}(\xi)}} d \xi,
$$

after the same change of variable. 


\subsection{The Gross-Pitaevskii nonlinearity}

In this section, we consider the Gross-Pitaevskii nonlinearity $f(\varrho)=1-\varrho$, that is

$$
i \frac{\partial \Psi}{\partial t}+\partial_{x}^{2} \Psi+\Psi\left(1-|\Psi|^{2}\right)=0
$$

We shall consider this model as a reference one, and this is why we detail some facts about it. For this particular nonlinearity, we have $r_{0}=1, \mathfrak{c}_{s}=\sqrt{2} \simeq 1.4142$, the functions $V$ and $\mathcal{V}$ are, respectively,

$$
V(\varrho)=\frac{1}{2}(\varrho-1)^{2}, \quad \mathcal{V}(\xi)=-2 \xi^{3}
$$

and the graphs of $f, V$ and $\mathcal{V}$ are

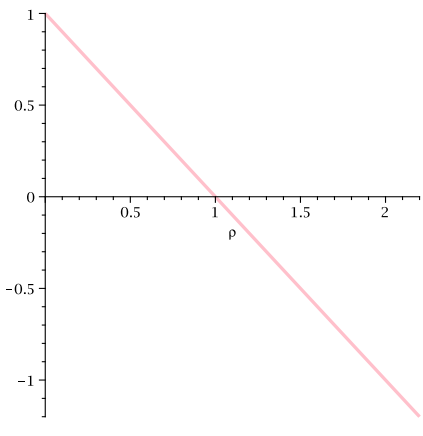

(a)

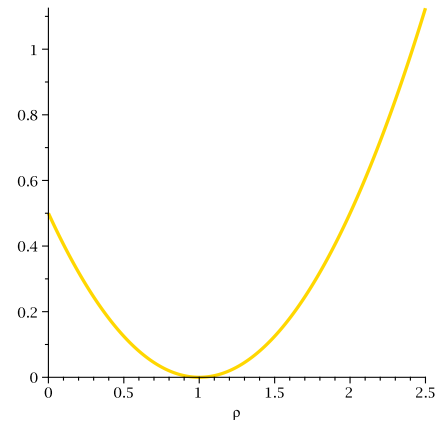

(b)

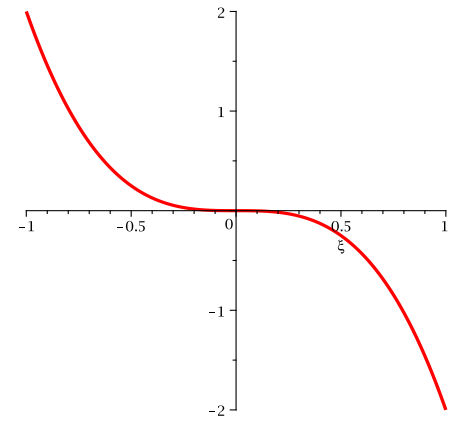

(c)

Figure 1: Graphs of (a) $f$, (b) $V$ and (c) $\mathcal{V}$.

Despite the fact that this model is widely used, it is also interesting since explicit computations of energy, momentum and traveling waves can be carried out. Indeed, we may compute explicitely the traveling waves for $0<c<\mathfrak{c}_{s}($ see [30], [6])

$$
U_{c}(x)=\sqrt{\frac{2-c^{2}}{2}} \tanh \left(x \frac{\sqrt{2-c^{2}}}{2}\right)-i \frac{c}{\sqrt{2}},
$$

which are unique up to translation or phase factor, and the energy and the momentum:

$$
E\left(U_{c}\right)=\frac{2}{3}\left(2-c^{2}\right)^{\frac{3}{2}} \quad P\left(U_{c}\right)=2 \arctan \left(\frac{\sqrt{2-c^{2}}}{c}\right)-c \sqrt{2-c^{2}} .
$$

Here are some representations of the potentials $\mathcal{V}_{c}$ for different values of $c$.

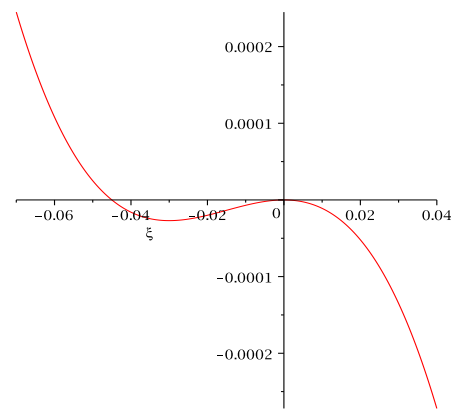

(a)

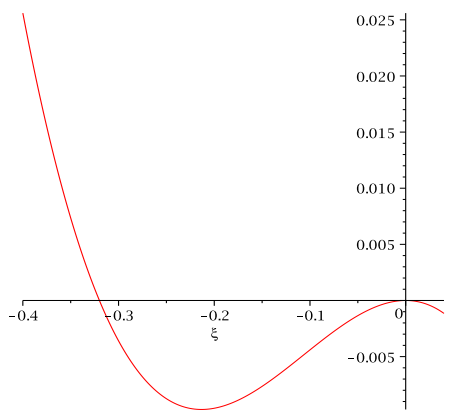

(b)

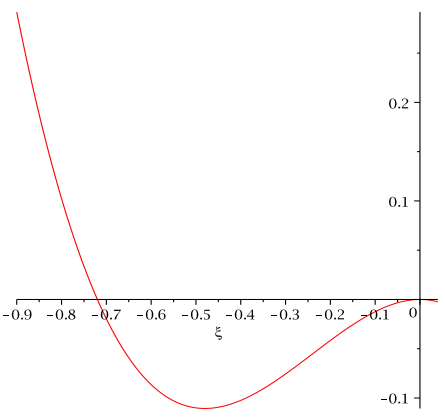

(c)

Figure 2: The potential $\mathcal{V}_{c}$ with $(\mathrm{a}) \varepsilon=0.3(c \simeq 1.3820) ;(\mathrm{b}) \varepsilon=0.8(c \simeq 1.1662) ;(\mathrm{c}) \varepsilon=1.2(c \simeq 0.7483)$. 
For $c \geq \mathfrak{c}_{s}$, in view of Theorem 1 , there does not exist nontrivial traveling waves. Plotting energy and momentum with respect to the speed and the energy-momentum diagram gives:

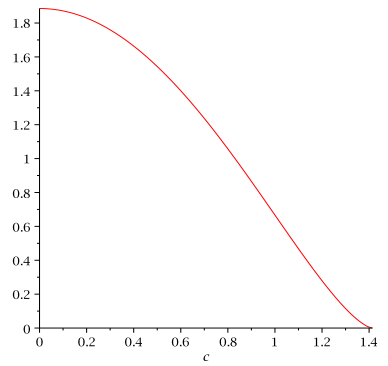

(a)

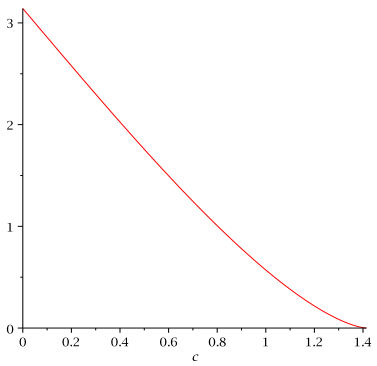

(b)

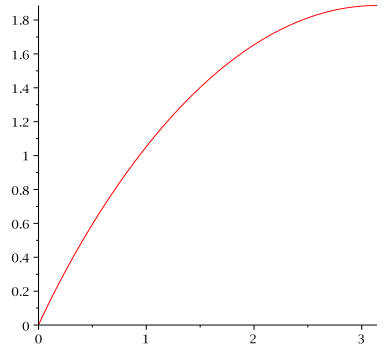

(c)

Figure 3: (a) Energy and (b) momentum vs. speed $c$; (c) (E,P) diagram.

In section 3, we shall study several model cases with a nonlinearity $f$ which is qualitatively as the Gross-Pitaevskii one in the sense that $f$ is smooth and decreases to minus infinity, but for which the energymomentum diagrams are very different from the (GP) one (figure $3(\mathrm{c})$ ).

\section{Mathematical results}

This section is devoted to mathematical results concerning the traveling waves for (NLS).

\subsection{Continuous dependence}

For the ODE $\left(\mathrm{TW}_{c}\right)$, we easily have a result of continuous dependence with respect to the parameter $c$. Indeed, if a nonconstant traveling wave $U_{*}$ exists for a speed $c_{*} \in\left(0, \mathfrak{c}_{s}\right)$, this means, working in hydrodynamical variables, that there exists an interval $\left(\xi_{*}, 0\right)$ (resp. $\left.\left(0, \xi_{*}\right)\right)$ on which $\mathcal{V}_{c_{*}}$ is negative and $\mathcal{V}_{c_{*}}\left(\xi_{*}\right)=0<\mathcal{V}_{c_{*}}^{\prime}\left(\xi_{*}\right)$ (resp. $\mathcal{V}_{c_{*}}\left(\xi_{*}\right)=0>\mathcal{V}_{c_{*}}^{\prime}\left(\xi_{*}\right)$ ). This is clearly not affected by a small perturbation of $c_{*}$, so that a branch of traveling waves $c \mapsto U_{c}$ exists near the traveling wave $U_{*}=U_{c_{*}}$, and we have locally uniqueness (recall that the invariance by translation is frozen by imposing $|U|$ to be even) in the sense that for $c$ close to $c_{*}$ and some $R>0$, there does not exist another traveling wave $u \neq U_{c}$ with $|u|$ even and $\left\|u-U_{c}\right\|_{L^{\infty}(\mathbb{R})} \leq R$. Moreover, it is easy to prove for $\eta_{c}$ and $u_{c}$ a uniform exponential decay for $c$ near $c_{*}$. By standard results on smooth dependence on the parameters for an ODE, if $f$ is smooth, $c \mapsto\left(\eta_{c}, u_{c}\right)$ is smooth with values into any Sobolev space $W^{s, p}(\mathbb{R}), s \in \mathbb{N}, 1 \leq p \leq \infty$ (we may also impose exponential decay). We can then show the standard Hamilton group relation (see e.g. [19])

$$
c_{*}=\frac{\partial E}{\partial P}{ }_{\mid c=c_{*}},
$$

where the derivative is taken along this branch, or more precisely

$$
\frac{d E}{d c}_{\mid c=c_{*}}=\left.c_{*} \frac{d P}{d c}\right|_{\mid c=c_{*}} .
$$

Indeed, due to the uniform exponential decay at infinity, we may differentiate

$$
\frac{d P\left(U_{c}\right)}{d c}=\frac{d}{d c} \int_{\mathbb{R}}\left(a_{c}^{2}-r_{0}^{2}\right) \partial_{x} \phi_{c} d x=\int_{\mathbb{R}} 2 a_{c} a_{c}^{\prime} \partial_{x} \phi_{c}+\left(a_{c}^{2}-r_{0}^{2}\right) \partial_{x} \phi_{c}^{\prime} d x
$$

where ' denotes differentiation with respect to $c$, and similarly

$$
\begin{aligned}
\frac{d E\left(U_{c}\right)}{d c} & =\int_{\mathbb{R}} 2 \partial_{x} a_{c} \partial_{x} a_{c}^{\prime}+2 a_{c} a_{c}^{\prime}\left(\partial_{x} \phi_{c}\right)^{2}+2 a_{c}^{2} \partial_{x} \phi_{c} \partial_{x} \phi_{c}^{\prime}-2 a_{c} a_{c}^{\prime} f\left(a_{c}^{2}\right) d x \\
& =\int_{\mathbb{R}} 2 a_{c}^{\prime}\left\{-\partial_{x}^{2} a_{c}-a_{c} f\left(a_{c}^{2}\right)+c a_{c} \partial_{x} \phi_{c}-a_{c}\left(\partial_{x} \phi_{c}\right)^{2}\right\}+2 a_{c}^{2} \partial_{x} \phi_{c} \partial_{x} \phi_{c}^{\prime}+2 c a_{c} a_{c}^{\prime} \partial_{x} \phi_{c} d x .
\end{aligned}
$$


The integration by parts is justified by the exponential decay of $a_{c}^{\prime}$ at infinity. Moreover, from $\left(\mathrm{TW}_{c}\right)$ we have

$$
\partial_{x}^{2} a_{c}-a_{c}\left(\partial_{x} \phi_{c}\right)^{2}+a_{c} f\left(a_{c}^{2}\right)+c a_{c} \partial_{x} \phi_{c}=0 \quad \text { and } \quad a_{c}^{2} \partial_{x} \phi_{c}=\frac{c}{2}\left(a_{c}^{2}-r_{0}^{2}\right) .
$$

Hence, the bracket term vanishes pointwise, and inserting the second equation yields

$$
\frac{d E\left(U_{c}\right)}{d c}=c \int_{\mathbb{R}}\left(a_{c}^{2}-r_{0}^{2}\right) \partial_{x} \phi_{c}^{\prime}+2 a_{c} a_{c}^{\prime} \partial_{x} \phi_{c} d x=c \frac{d P\left(U_{c}\right)}{d c}
$$

as required.

The relation (12) imposes that critical points of the functions $c \mapsto E$ and $c \mapsto P$ (such as local maxima or local minima), occur at the same time (for $c \neq 0$ ). Moreover, this also forces $\frac{d E}{d c}$ to vanish for $c \rightarrow 0$. These two points can be cheked on the various model cases given in section 3 .

\subsection{Stability}

In order to study the orbital stability or instability of these traveling waves, one may use a result of Z. Lin (see [24]), which shows the orbital stability under the assumption

$$
\frac{d P}{d c}<0
$$

and instability under the hypothesis

$$
\frac{d P}{d c}>0
$$

Here, the derivative is taken along the (local) branch. This result establishes rigorously the stability criterion found in [8], [3]. On the energy-momentum diagram, this criterion reads as follows: if, on the local branch, $P \mapsto E$ is concave in the sense that $\frac{d^{2} E}{d P^{2}}<0$, then the traveling wave is orbitally stable; and if $P \mapsto E$ is convex in the sense that $\frac{d^{2} E}{d P^{2}}>0$, then the traveling wave is orbitally unstable. This point follows immediately from the Hamilton group relation (12). Indeed, we have

$$
\frac{d^{2} E}{d P^{2}}=\frac{d}{d P}\left(\frac{d E}{d P}\right)=\frac{d c}{d P}
$$

The result in [24] is proved for a nonlinearity for which we have existence of traveling waves for any $c \in\left[0, \mathfrak{c}_{s}\right)$, but the arguments work for the nonlinearities $f$ we are considering. Indeed, the analysis extends the results of [9], [29], [17] and relies on some spectral properties of the linearized problem, for which Sturm-Liouville theory still gives the existence of some simple negative eigenvalue associated to a positive function. As a first step, we recall a local well-posedness result in the Zhidkov space

$$
\mathcal{Z}^{1} \equiv\left\{v \in L^{\infty}(\mathbb{R}), \partial_{x} v \in L^{2}(\mathbb{R}),|v|^{2}-r_{0}^{2} \in L^{2}(\mathbb{R})\right\}
$$

due to P. Zhidkov [32] (see also [13] and [14] for global well-posedness results).

Theorem $2([32])$ Let $\Psi^{\text {in }} \in \mathcal{Z}^{1}$. Then, there exists $T_{*}>0$ and a unique solution $\Psi$ to (NLS) such that $\Psi_{\mid t=0}=\Psi^{\text {in }}$ and $\Psi-\Psi^{\text {in }} \in \mathcal{C}\left(\left[0, T_{*}\right), H^{1}(\mathbb{R})\right)$. Moreover, $E(\Psi(t))$ does not depend on $t$.

We can now state the stability/instability result of [24].

Theorem 3 ([24]) Assume that $0<c_{*}<\mathfrak{c}_{s}$ is such that there exists a nontrivial traveling wave $U_{c_{*}}$. Then, there exists some small $\sigma>0$ such that $U_{c_{*}}$ belongs to a locally unique continuous branch of nontrivial traveling waves $U_{c}$ defined for $c_{*}-\sigma \leq c \leq c_{*}+\sigma$.

(i) Assume

$$
\frac{d P\left(U_{c}\right)}{d c}{ }_{\mid c=c_{*}}<0
$$


Then, $U_{c_{*}}=a_{*} \mathrm{e}^{i \phi_{*}}$ is orbitally stable in the sense that for any $\epsilon>0$, there exists $\delta>0$ such that if $\Psi^{\text {in }}=a^{\text {in }} \mathrm{e}^{i \phi^{\text {in }}} \in \mathcal{Z}^{1}$ verifies ${ }^{1}$

$$
\left\|a^{\text {in }}-a_{*}\right\|_{H^{1}(\mathbb{R})}+\left\|\partial_{x} \phi^{\text {in }}-\partial_{x} \phi_{*}\right\|_{L^{2}(\mathbb{R})} \leq \delta
$$

then, the solution $\Psi$ to (NLS) such that $\Psi_{\mid t=0}=\Psi^{\text {in }}$ never vanishes, can be lifted $\Psi=$ a $\mathrm{e}^{i \phi}$, and we have

$$
\sup _{t \geq 0} \inf _{y \in \mathbb{R}}\left\{\left\|a(t)-a_{*}(\cdot-y)\right\|_{H^{1}(\mathbb{R})}+\left\|\partial_{x} \phi(t)-\partial_{x} \phi_{*}(\cdot-y)\right\|_{L^{2}(\mathbb{R})}\right\} \leq \epsilon .
$$

(ii) Assume

$$
{\frac{d P\left(U_{c}\right)}{d c} \mid c=c_{*}}>0 .
$$

Then, $U_{c_{*}}=a_{*} \mathrm{e}^{i \phi_{*}}$ is orbitally unstable in the sense that there exists $\epsilon>0$ such that, for any $\delta>0$, there exists $\Psi^{\text {in }}=a^{\text {in }} \mathrm{e}^{i \phi^{\text {in }}} \in \mathcal{Z}^{1}$ verifying

$$
\left\|a^{\text {in }}-a_{*}\right\|_{H^{1}(\mathbb{R})}+\left\|\partial_{x} \phi^{\text {in }}-\partial_{x} \phi_{*}\right\|_{L^{2}(\mathbb{R})} \leq \delta
$$

but such that if $\Psi$ denotes the solution to (NLS) with $\Psi_{\mid t=0}=\Psi^{\mathrm{in}}$, then there exists $t>0$ such that $\Psi$ does not vanish on the time interval $[0, t]$ but

$$
\inf _{y \in \mathbb{R}}\left\{\left\|a(t)-a_{*}(\cdot-y)\right\|_{H^{1}(\mathbb{R})}+\left\|\partial_{x} \phi(t)-\partial_{x} \phi_{*}(\cdot-y)\right\|_{L^{2}(\mathbb{R})}\right\} \geq \epsilon
$$

In particular, for the Gross-Pitaevskii nonlinearity, Theorem 3 shows that the traveling waves with speed $c \in\left(0, \mathfrak{c}_{s}\right)$ are orbitally stable, since the energy-momentum diagram is strictly concave. This was also shown in [6] using a variational characterization of these traveling waves, namely that they minimize the energy at fixed momentum.

Remark 1 Theorem 3 does not work for $c=\mathfrak{c}_{s}$, since the spectral decomposition in [24] is then no longer true, hence it is not clear to know what happens for sonic traveling waves, if they exist. Furthermore, the above result does not say anything in the degenerate case $\frac{d P}{d c}=0$.

Concerning the stationnary traveling wave solutions $(c=0)$, we quote the paper [11] for instability of the bubble ( $U$ is even and does not vanish). Concerning the kink ( $U$ is odd), the paper [12] gives a linear stability criterion through the so called Vakhitov-Kolokolov function, and proves nonlinear instability when linear instability holds, justifying that a kink can be unstable (as was suggested for the first time by [21] for the saturated (NLS)). Note that the approach of [24], that is Theorem 3, relies on the hydrodynamical formulation of (NLS), hence can not be used for the kink. For the Gross-Pitaevskii nonlinearity, the kink was shown to be stable in [7] using a variational characterization (the kink minimizes the energy with a suitable constraint on the momentum), and in the paper [15] in a different functional space using inverse scattering. In some forthcoming work, we shall give some results on the stability of the traveling waves in the cases left open by Theorem 3 .

\subsection{Transonic limit}

In the transonic limit $c \rightarrow \mathfrak{c}_{s}$, the traveling waves are expected to be close, up to rescaling, to the (KdV) soliton. The formal derivation is as follows (see [31], [20] and [4] for the time dependent derivation, useful for the analysis of modulations). This corresponds to the case where

$$
\varepsilon \equiv \sqrt{\mathfrak{c}_{s}^{2}-c^{2}}
$$

is small. We insert the ansatz

$$
U(x)=r_{0}\left(1+\varepsilon^{2} A_{\varepsilon}(z)\right) \exp \left(i \varphi_{\varepsilon}(z)\right) \quad z \equiv \varepsilon x
$$

\footnotetext{
${ }^{1}$ From the embedding $H^{1}(\mathbb{R}) \hookrightarrow L^{\infty}(\mathbb{R})$, since $U_{\mathcal{C}_{*}}$ does not vanish and $\left|\Psi^{\text {in }}\right|$ is close in $H^{1}(\mathbb{R})$ to $\left|U_{c_{*}}\right|$ by the first term, $\Psi^{\text {in }}$ does not vanish and can be lifted: $\Psi^{\text {in }}=a^{\text {in }} e^{i \phi^{\text {in }}}$
} 
into (2):

$$
\left\{\begin{array}{l}
-\sqrt{\mathfrak{c}_{s}^{2}-\varepsilon^{2}} \partial_{z} A_{\varepsilon}+2 \varepsilon^{2} \partial_{z} \varphi_{\varepsilon} \partial_{z} A_{\varepsilon}+\left(1+\varepsilon^{2} A_{\varepsilon}\right) \partial_{z}^{2} \varphi_{\varepsilon}=0 \\
-\sqrt{\mathfrak{c}_{s}^{2}-\varepsilon^{2}} \partial_{z} \varphi_{\varepsilon}+\varepsilon^{2}\left(\partial_{z} \varphi_{\varepsilon}\right)^{2}-\frac{1}{\varepsilon^{2}} f\left(r_{0}^{2}\left(1+\varepsilon^{2} A_{\varepsilon}\right)^{2}\right)-\varepsilon^{2} \frac{\partial_{z}^{2} A_{\varepsilon}}{1+\varepsilon^{2} A_{\varepsilon}}=0
\end{array}\right.
$$

Moreover, Taylor expansion gives

$$
f\left(r_{0}^{2}(1+\alpha)^{2}\right)=-\mathfrak{c}_{s}^{2} \alpha+\left(-\frac{\mathfrak{c}_{s}^{2}}{2}+2 f^{\prime \prime}\left(r_{0}^{2}\right)\right) \alpha^{2}+f_{3}(\alpha),
$$

with $f_{3}(\alpha)=\mathcal{O}\left(\alpha^{3}\right)$ as $\alpha \rightarrow 0$. If $\varphi_{\varepsilon} \rightarrow \varphi$ and $A_{\varepsilon} \rightarrow A$ in some suitable sense, both equations of (14) give to leading order the constraint

$$
\partial_{z} \varphi=\mathfrak{c}_{s} A .
$$

We now add $\sqrt{\mathfrak{c}_{s}^{2}-\varepsilon^{2}} / \mathfrak{c}_{s}^{2}$ times the first equation of (14) and $\partial_{z_{1}} / \mathfrak{c}_{s}^{2}$ times the second one and divide by $\varepsilon^{2}$ to deduce

$$
\begin{array}{r}
\frac{1}{\mathfrak{c}_{s}^{2}} \partial_{z} A_{\varepsilon}+\left\{2 \frac{\sqrt{\mathfrak{c}_{s}^{2}-\varepsilon^{2}}}{\mathfrak{c}_{s}^{2}} \partial_{z} \varphi_{\varepsilon} \partial_{z} A_{\varepsilon}+\frac{\sqrt{\mathfrak{c}_{s}^{2}-\varepsilon^{2}}}{\mathfrak{c}_{s}^{2}} A_{\varepsilon} \partial_{z}^{2} \varphi_{\varepsilon}+\right. \\
\left.+\frac{1}{\mathfrak{c}_{s}^{2}} \partial_{z}\left[\left(\partial_{z} \varphi_{\varepsilon}\right)^{2}\right]+\left[\frac{1}{2}-2 r_{0}^{4} \frac{f^{\prime \prime}\left(r_{0}^{2}\right)}{\mathfrak{c}_{s}^{2}}\right] \partial_{z}\left(A_{\varepsilon}^{2}\right)\right\} \\
-\frac{1}{\mathfrak{c}_{s}^{2}} \partial_{z}\left(\frac{\partial_{z}^{2} A_{\varepsilon}}{1+\varepsilon^{2} A_{\varepsilon}}\right)=-\frac{1}{\mathfrak{c}_{s}^{2} \varepsilon^{4}} \partial_{z}\left[f_{3}\left(\varepsilon^{2} A_{\varepsilon}\right)\right] .
\end{array}
$$

Passing to the formal limit $\varepsilon \rightarrow 0$ and using (15), we infer

$$
\frac{1}{\mathfrak{c}_{s}^{2}} \partial_{z} A+\Gamma A \partial_{z} A-\frac{1}{\mathfrak{c}_{s}^{2}} \partial_{z}^{3} A=0, \quad \text { with } \quad \Gamma \equiv 6-\frac{4 r_{0}^{4}}{\mathfrak{c}_{s}^{2}} f^{\prime \prime}\left(r_{0}^{2}\right) .
$$

This is the (KdV) solitary wave equation, for which the only nontrivial solution is, up to a space translation,

$$
\mathrm{w}(z) \equiv-\frac{3}{\mathfrak{c}_{s}^{2} \Gamma \cosh ^{2}(z / 2)} .
$$

For instance, for the Gross-Pitaevskii equation we have $f(\varrho)=1-\varrho, \mathfrak{c}_{s}^{2}=2, \Gamma=6$ and the explicit formula (11) implies, with $2-c^{2}=\varepsilon^{2}$,

$$
\left|U_{c}\right|^{2}(x)=\frac{\varepsilon^{2}}{2} \tanh ^{2}\left(\frac{\varepsilon x}{2}\right)+\frac{2-\varepsilon^{2}}{2}=1-\frac{\varepsilon^{2}}{2 \cosh ^{2}(\varepsilon x / 2)},
$$

so that

$$
\left(1+\varepsilon^{2} A_{\varepsilon}(z=\varepsilon x)\right)^{2}=\left|U_{c}\right|^{2}(x)=1+2 \varepsilon^{2} \mathrm{w}(\varepsilon x)
$$

and thus the convergence of $A_{\varepsilon}$ to w follows.

For a general nonlinearity $f$, we have the following result.

Theorem 4 We assume $f \in \mathcal{C}^{n}\left(\mathbb{R}_{+}, \mathbb{R}\right)$ for some $n \geq 3$, and $\Gamma \neq 0$. Then, there exists $\delta>0$ and $0<\varepsilon_{0}<\mathfrak{c}_{s}$ with the following properties. For any $0<\varepsilon \leq \varepsilon_{0}$ (or, equivalently, $\mathfrak{c}_{0} \equiv c\left(\varepsilon_{0}\right) \leq c(\varepsilon)<\mathfrak{c}_{s}$ ), there exists a solution $U_{c(\varepsilon)}$ to $\left(\mathrm{TW}_{c(\varepsilon)}\right)$ satisfying $\left\|\left|U_{c(\varepsilon)}\right|-r_{0}\right\|_{L^{\infty}(\mathbb{R})} \leq \delta$. If $0<\varepsilon<\varepsilon_{0}$ and if $u$ is a nonconstant traveling wave of speed $c(\varepsilon)$ verifying $\left\||u|-r_{0}\right\|_{L^{\infty}(\mathbb{R})} \leq \delta$, then there exists $\theta \in \mathbb{R}$ and $y \in \mathbb{R}$ such that $u(x)=\mathrm{e}^{i \theta} U_{c(\varepsilon)}(x-y)$. The map $U_{c(\varepsilon)}$ can be written

$$
U_{c(\varepsilon)}(x)=r_{0}\left(1+\varepsilon^{2} A_{\varepsilon}(z)\right) \exp \left(i \varepsilon \varphi_{\varepsilon}(z)\right), \quad z \equiv \varepsilon x,
$$

and for any $s \in \mathbb{N}, 0 \leq s \leq n+2$ and $1 \leq p \leq \infty$,

$$
\partial_{z} \varphi_{\varepsilon} \rightarrow \mathfrak{c}_{s} \mathrm{~W} \quad \text { and } \quad A_{\varepsilon} \rightarrow \mathrm{w} \quad \text { in } W^{s, p}(\mathbb{R}) \quad \text { as } \varepsilon \rightarrow 0 .
$$


Finally, as $\varepsilon \rightarrow 0$,

$$
E\left(U_{c(\varepsilon)}\right) \sim \mathfrak{c}_{s} P\left(U_{c(\varepsilon)}\right) \sim \frac{48 r_{0}^{2}}{\mathfrak{c}_{s}^{2} \Gamma^{2}}\left(\mathfrak{c}_{s}^{2}-c^{2}(\varepsilon)\right)^{\frac{3}{2}}=\varepsilon^{3} \frac{48 r_{0}^{2}}{\mathfrak{c}_{s}^{2} \Gamma^{2}}
$$

and

$$
E\left(U_{c(\varepsilon)}\right)-c(\varepsilon) P\left(U_{c(\varepsilon)}\right) \sim \frac{48 r_{0}^{2}}{5 \mathfrak{c}_{s}^{4} \Gamma^{2}}\left(\mathfrak{c}_{s}^{2}-c^{2}(\varepsilon)\right)^{\frac{5}{2}}=\frac{48 r_{0}^{2}}{5 \mathfrak{c}_{s}^{4} \Gamma^{2}} \varepsilon^{5} .
$$

Proof. The potential function $\mathcal{V}$ has the Taylor expansion near $\xi=0$ :

$\mathcal{V}(\xi)=\mathfrak{c}_{s}^{2} \xi^{2}-4\left(r_{0}^{2}+\xi\right) V\left(r_{0}^{2}+\xi\right)=\mathfrak{c}_{s}^{2} \xi^{2}-4\left(r_{0}^{2}+\xi\right)\left(-\frac{1}{2} f^{\prime}\left(r_{0}^{2}\right) \xi^{2}-\frac{1}{6} f^{\prime \prime}\left(r_{0}^{2}\right) \xi^{3}+\mathcal{O}\left(\xi^{4}\right)\right)=-\frac{\mathfrak{c}_{s}^{2} \Gamma}{6 r_{0}^{2}} \xi^{3}+\mathcal{O}\left(\xi^{4}\right)$,

by definition of $\mathfrak{c}_{s}$ and $\Gamma$. Therefore, since $\Gamma \neq 0$ by hypothesis,

$$
\mathcal{V}_{c(\varepsilon)}(\xi)=\mathcal{V}(\xi)-\varepsilon^{2} \xi^{2}=-\varepsilon^{2} \xi^{2}-\frac{\mathfrak{c}_{s}^{2} \Gamma}{6 r_{0}^{2}} \xi^{3}+\mathcal{O}\left(\xi^{4}\right) \quad \text { as } \quad \xi \rightarrow 0
$$

Then, with $\Gamma>0$ for instance and for small $\varepsilon>0$, the potential function $\mathcal{V}_{c(\varepsilon)}$ has the following graph:

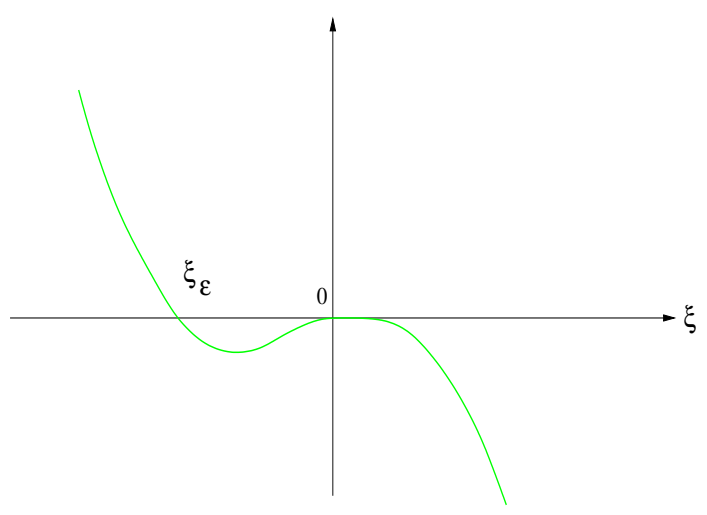

Figure 4: Graph of $\mathcal{V}(\xi)-\varepsilon^{2} \xi^{2}$.

The first negative zero $\xi_{\varepsilon}$ of $\mathcal{V}_{c(\varepsilon)}$ has the expansion

$$
\xi_{\varepsilon}=-\frac{6 r_{0}^{2}}{\mathfrak{c}_{s}^{2} \Gamma} \varepsilon^{2}+\mathcal{O}\left(\varepsilon^{3}\right)
$$

For $\varepsilon>0$ small, the function $\mathcal{V}_{c(\varepsilon)}$ is negative in $\left(\xi_{\varepsilon}, 0\right)$ and $\xi_{\varepsilon}$ is a simple zero: there exists a continuous branch of traveling waves $U_{c(\varepsilon)}$ for $\varepsilon>0$ small enough, and there holds

$$
\xi_{\varepsilon} \leq\left|U_{c(\varepsilon)}\right|^{2}-r_{0}^{2}=\eta_{c(\varepsilon)} \leq 0
$$

in $\mathbb{R}$. On the other hand, $\mathcal{V}$ is negative in some interval $\left(0, \xi_{*}\right)$ with $\xi_{*}>0$ (and possibly $\xi_{*}=+\infty$ ). Hence, if $u$ is a nontrivial traveling wave with $|u| \geq r_{0}$ and $\varepsilon$ small, then $\eta=|u|^{2}-r_{0}^{2}$ has to reach values $\geq \xi_{*}$, and then $\left\||u|-r_{0}\right\|_{L^{\infty}(\mathbb{R})} \geq \delta$ for some $\delta>0$. Moreover, it comes from (10) that, for $x \geq 0$,

$$
x=\int_{\xi_{\varepsilon}}^{\eta_{c(\varepsilon)}(x)} \frac{d \xi}{\sqrt{-\mathcal{V}_{c(\varepsilon)}(\xi)}} .
$$

We now scale

$$
U_{c(\varepsilon)}(x)=r_{0} \sqrt{1+\varepsilon^{2} \mathcal{A}_{\varepsilon}(z)} \exp \left(i \varepsilon \varphi_{\varepsilon}(z)\right), \quad z=\varepsilon x .
$$

The way we write the amplitude is slightly different from (13), but $A_{\varepsilon}$ and $\mathcal{A}_{\varepsilon}$ are related by the formulas

$$
\mathcal{A}_{\varepsilon}=2 A_{\varepsilon}+\varepsilon^{2} A_{\varepsilon}^{2} \quad \text { or } \quad A_{\varepsilon}=\frac{\sqrt{1+\varepsilon^{2} \mathcal{A}_{\varepsilon}}-1}{\varepsilon^{2}}=\frac{\mathcal{A}_{\varepsilon}}{1+\sqrt{1+\varepsilon^{2} \mathcal{A}_{\varepsilon}}} .
$$


This way of writting the amplitude is well-adapted to the Newton equation on $\eta=|U|^{2}-r_{0}^{2}$, which involves the density $|U|^{2}$. Moreover, it is clear that in order to show compactness on $A_{\varepsilon}$, it suffices to show compactness on $\mathcal{A}_{\varepsilon}$ in $\mathbb{R}_{+}$(this is an even function), which will be done by using Ascoli's theorem. Then, we immediately have

$$
-\frac{6}{\mathfrak{c}_{s}^{2} \Gamma}+\mathcal{O}(\varepsilon)=\frac{\xi_{\varepsilon}}{r_{0}^{2} \varepsilon^{2}} \leq \mathcal{A}_{\varepsilon}(z)=\frac{\eta_{c(\varepsilon)}(z / \varepsilon)}{\varepsilon^{2} r_{0}^{2}} \leq 0
$$

and, from (17), with $\xi=r_{0}^{2} \varepsilon^{2} \zeta$ and for $z \geq 0$,

$$
z=\int_{\xi_{\varepsilon} /\left(r_{0}^{2} \varepsilon^{2}\right)}^{\mathcal{A}_{\varepsilon}(z)} \frac{d \zeta}{\sqrt{\zeta^{2}+\frac{\mathfrak{c}_{s}^{2} \Gamma}{6} \zeta^{3}+\mathcal{O}\left(\varepsilon^{2} \zeta^{4}\right)}}
$$

Here, the " $\mathcal{O}\left(\varepsilon^{2} \zeta^{4}\right)$ " is uniform in $\zeta$ (which remains in a compact set independent of $\varepsilon$ small). Notice that as $\mathcal{A} \rightarrow 0^{-}$,

$$
\begin{aligned}
& \int_{\xi_{\varepsilon} /\left(r_{0}^{2} \varepsilon^{2}\right)}^{\mathcal{A}} \frac{d \zeta}{\sqrt{\zeta^{2}+\frac{\mathfrak{c}_{s}^{2} \Gamma}{6} \zeta^{3}+\mathcal{O}\left(\varepsilon^{2} \zeta^{4}\right)}} \\
&=\int_{\xi_{\varepsilon} /\left(r_{0}^{2} \varepsilon^{2}\right)}^{\frac{1}{c_{c}^{2} \Gamma}} \frac{d \zeta}{\sqrt{\zeta^{2}+\frac{\mathfrak{c}_{s}^{2} \Gamma}{6} \zeta^{3}+\mathcal{O}\left(\varepsilon^{2} \zeta^{4}\right)}}-\int_{\frac{1}{\mathfrak{c}_{s}^{2} \Gamma}}^{\mathcal{A}} \frac{d \zeta}{\zeta \sqrt{1+\frac{\mathfrak{c}_{s}^{2} \Gamma}{6} \zeta+\mathcal{O}\left(\varepsilon^{2} \zeta^{2}\right)}}=-\ln |\mathcal{A}|+\mathcal{O}(1)
\end{aligned}
$$

uniformly in $\varepsilon$ (sufficiently small). It then follows that for some constant $C>0$, we have

$$
-C \mathrm{e}^{-|z|} \leq \mathcal{A}_{\varepsilon}(z) \leq 0
$$

and, from (10), a similar estimate holds for $\partial_{z} \mathcal{A}_{\varepsilon}$. It is then possible to pass to the limit (using Ascoli's theorem and the uniform exponential decay) to infer that for some sequence $\varepsilon_{j} \rightarrow 0, \mathcal{A}_{\varepsilon_{j}}$ converges to some $\mathcal{A}$ uniformly in $\mathbb{R}$ such that

$$
z=\int_{-6 /\left(\mathfrak{c}_{s}^{2} \Gamma\right)}^{\mathcal{A}(z)} \frac{d \zeta}{\sqrt{\zeta^{2}+\frac{\mathfrak{c}_{s}^{2} \Gamma}{6} \zeta^{3}}} .
$$

Hence $\mathcal{A}=2 \mathrm{w}$, where $\mathrm{w}$ is the $(\mathrm{KdV})$ soliton. From the uniqueness of the limit, we deduce that the full familly $\left(\mathcal{A}_{\varepsilon}\right)_{\varepsilon>0}$ converges to $2 \mathrm{w}$. Taking the derivatives of the first integral (9), it is easy to infer that all the derivatives of $\mathcal{A}_{\varepsilon}$ satisfy some uniform exponential decay, hence the convergence of $\mathcal{A}_{\varepsilon}$ to $2 \mathrm{w}$ in the Sobolev spaces $W^{s, p}(\mathbb{R}), s \in \mathbb{N}, 0 \leq s \leq n+2,1 \leq p \leq \infty$ follows. The convergence of $A_{\varepsilon}$ follows from the formula (18) and the convergence for the derivative of the phase follows from equation (6), which rescales as

$$
\partial_{z} \varphi_{\varepsilon}=c(\varepsilon) \frac{\mathcal{A}_{\varepsilon}}{2\left(1+\varepsilon^{2} \mathcal{A}_{\varepsilon}\right)} .
$$

For the convergences, the proof is complete when $\Gamma>0$, and the case $\Gamma<0$ is analoguous. It remains to compute the asymptotic behaviour of the energy and momentum, which are of course related to the ones of the $(\mathrm{KdV})$ soliton w. Indeed, by using the variable $\xi=\varepsilon^{2} r_{0}^{2} \zeta$ one has

$$
\begin{aligned}
P\left(U_{c(\varepsilon)}\right) & =\operatorname{sgn}\left(\xi_{\varepsilon}\right) c(\varepsilon) \int_{0}^{\xi_{\varepsilon}} \frac{\xi^{2}}{r_{0}^{2}+\xi} \frac{d \xi}{\sqrt{\varepsilon^{2} \xi^{2}-\mathcal{V}(\xi)}} \\
& =\operatorname{sgn}\left(\xi_{\varepsilon}\right) r_{0}^{2} \varepsilon^{3} c(\varepsilon) \int_{0}^{\xi_{\varepsilon} /\left(\varepsilon^{2} r_{0}^{2}\right)} \frac{\zeta^{2}}{1+\varepsilon^{2} \zeta} \frac{d \zeta}{\sqrt{\zeta^{2}+\frac{\mathfrak{c}_{s}^{2} \Gamma}{6} \zeta^{3}+\mathcal{O}\left(\varepsilon^{2} \zeta^{4}\right)}} \\
& \sim r_{0}^{2} \varepsilon^{3} \mathfrak{c}_{s} \int_{0}^{-6 /\left(\mathfrak{c}_{s}^{2} \Gamma\right)} \frac{\zeta d \zeta}{\sqrt{1+\frac{\mathfrak{c}_{s}^{2} \Gamma}{6} \zeta}}=r_{0}^{2} \varepsilon^{3} \frac{48}{\mathfrak{c}_{s}^{3} \Gamma^{2}}
\end{aligned}
$$


and the computation for the energy is similar. Finally, from the expressions of energy and momentum given in subsection 1.2, the definition of $\mathcal{V}_{c}$, and using the variable $\xi=\varepsilon^{2} r_{0}^{2} \zeta$, it follows,

$$
\begin{aligned}
E\left(U_{c(\varepsilon)}\right)-c(\varepsilon) P\left(U_{c(\varepsilon)}\right) & =\operatorname{sgn}\left(\xi_{\varepsilon}\right) \int_{0}^{\xi_{\varepsilon}} \frac{\sqrt{-\mathcal{V}(\xi)}}{r_{0}^{2}+\xi} d \xi \\
& =\operatorname{sgn}\left(\xi_{\varepsilon}\right) \varepsilon^{5} r_{0}^{4} \int_{0}^{\xi_{\varepsilon} /\left(\varepsilon^{2} r_{0}^{2}\right)} \frac{\sqrt{\zeta^{2}+\frac{\mathfrak{c}_{s}^{2} \Gamma}{6} \zeta^{3}+\mathcal{O}\left(\varepsilon^{2} \zeta^{4}\right)}}{1+\varepsilon^{2} \zeta} d \zeta \\
& \sim \varepsilon^{5} r_{0}^{4} \int_{0}^{-6 /\left(\mathfrak{c}_{s}^{2} \Gamma\right)} \sqrt{\zeta^{2}+\frac{\mathfrak{c}_{s}^{2} \Gamma}{6} \zeta^{3}} d \zeta=\frac{48 r_{0}^{2}}{5 \mathfrak{c}_{s}^{4} \Gamma^{2}} \varepsilon^{5} .
\end{aligned}
$$

The proof is complete.

So far, the degenerate case $\Gamma=0$ has not been thoroughly investigated. We would like to emphasize that the coefficient $\Gamma$ involves the second order derivative of $f$ at $\varrho=r_{0}^{2}$. Even though the case $\Gamma=0$ is not generic, we shall see that the qualitative behaviour of the traveling waves to (NLS) can be extremely different from the well-known Gross-Pitaevskii case $f(\varrho)=1-\varrho$. The coefficient $\Gamma$ is actually linked to the function $\mathcal{V}$ appearing in (9) by the equality

$$
\mathcal{V}^{(3)}(0)=12 f^{\prime}\left(r_{0}^{2}\right)+4 r_{0}^{2} f^{\prime \prime}\left(r_{0}^{2}\right)=-\frac{\Gamma \mathfrak{c}_{s}^{2}}{r_{0}^{2}} .
$$

When $\Gamma=0$, the nonlinear term in the $(\mathrm{KdV})$ solitary wave (16) disappears, and there is no soliton. In order to see the nonlinear terms, we have to assume $\left(A_{\varepsilon}, \varphi_{\varepsilon}\right)$ larger and expand further the nonlinearity. We thus make the ansatz

$$
U(x)=r_{0}\left(1+\varepsilon A_{\varepsilon}(z)\right) \exp \left(i \varphi_{\varepsilon}(z)\right) \quad z \equiv \varepsilon x,
$$

plug this into (2) and obtain

$$
\left\{\begin{array}{l}
-c(\varepsilon) \partial_{z} A_{\varepsilon}+2 \varepsilon \partial_{z} \varphi_{\varepsilon} \partial_{z} A_{\varepsilon}+\left(1+\varepsilon A_{\varepsilon}\right) \partial_{z}^{2} \varphi_{\varepsilon}=0 \\
-c(\varepsilon) \partial_{z} \varphi_{\varepsilon}+\varepsilon\left(\partial_{z} \varphi_{\varepsilon}\right)^{2}-\frac{1}{\varepsilon} f\left(r_{0}^{2}\left(1+\varepsilon A_{\varepsilon}\right)^{2}\right)-\varepsilon^{2} \frac{\partial_{z}^{2} A_{\varepsilon}}{1+\varepsilon A_{\varepsilon}}=0
\end{array}\right.
$$

Here again, if $A_{\varepsilon} \rightarrow A$ and $\varphi_{\varepsilon} \rightarrow \varphi$ as $\varepsilon \rightarrow 0$, we infer that at leading order, for both equations, the constraint (15) holds. However, we shall need a second order expansion: we thus write the Taylor expansion

$$
f\left(r_{0}^{2}(1+\alpha)^{2}\right)=-\mathfrak{c}_{s}^{2} \alpha-\left(\frac{\mathfrak{c}_{s}^{2}}{2}-2 r_{0}^{4} f^{\prime \prime}\left(r_{0}^{2}\right)\right) \alpha^{2}+\left(2 r_{0}^{2} f^{\prime \prime}\left(r_{0}^{2}\right)+\frac{4}{3} r_{0}^{6} f^{\prime \prime \prime}\left(r_{0}^{2}\right)\right) \alpha^{3}+f_{4}(\alpha),
$$

with $f_{4}(\alpha)=\mathcal{O}\left(\alpha^{4}\right)$ as $\alpha \rightarrow 0$, and keep the terms of order $\varepsilon^{0}$ and $\varepsilon^{1}$ in (20). Since $c^{2}(\varepsilon)=\mathfrak{c}_{s}^{2}-\varepsilon^{2}$, we get

$$
\left\{\begin{array}{l}
\partial_{z}^{2} \varphi_{\varepsilon}-c(\varepsilon) \partial_{z} A_{\varepsilon}+2 \varepsilon \partial_{z} \varphi_{\varepsilon} \partial_{z} A_{\varepsilon}+\varepsilon A_{\varepsilon} \partial_{z}^{2} \varphi_{\varepsilon}=\mathcal{O}\left(\varepsilon^{2}\right) \\
c^{2}(\varepsilon) A_{\varepsilon}-c(\varepsilon) \partial_{z} \varphi_{\varepsilon}+\varepsilon\left(\partial_{z} \varphi_{\varepsilon}\right)^{2}+\varepsilon\left(\frac{\mathfrak{c}_{s}^{2}}{2}-2 r_{0}^{4} f^{\prime \prime}\left(r_{0}^{2}\right)\right) A_{\varepsilon}^{2}=\mathcal{O}\left(\varepsilon^{2}\right)
\end{array}\right.
$$

Since $\Gamma=0$, we have

$$
2 r_{0}^{4} f^{\prime \prime}\left(r_{0}^{2}\right)=3 c_{s}^{2}
$$

Therefore, each of the two equations in the above system reduce to

$$
\partial_{z} \varphi_{\varepsilon}-c(\varepsilon) A_{\varepsilon}=\partial_{z} \varphi_{\varepsilon}-\mathfrak{c}_{s} A_{\varepsilon}+\mathcal{O}\left(\varepsilon^{2}\right)=-\frac{3 \varepsilon}{2} \mathfrak{c}_{s} A_{\varepsilon}^{2}+\mathcal{O}\left(\varepsilon^{2}\right)
$$

Adding $c(\varepsilon) / \mathfrak{c}_{s}^{2}$ times the first equation of $(20)$ and $\partial_{z} / \mathfrak{c}_{s}^{2}$ times the second one and dividing by $\varepsilon^{2}$, we get

$$
\begin{aligned}
\frac{1}{\mathfrak{c}_{s}^{2}} \partial_{z} A_{\varepsilon} & -\frac{1}{\mathfrak{c}_{s}^{2}} \partial_{z}\left(\frac{\partial_{z}^{2} A_{\varepsilon}}{1+\varepsilon A_{\varepsilon}}\right)-\frac{1}{\mathfrak{c}_{s}^{2}}\left(6 r_{0}^{2} f^{\prime \prime}\left(r_{0}^{2}\right)+4 r_{0}^{6} f^{\prime \prime \prime}\left(r_{0}^{2}\right)\right) A_{\varepsilon}^{2} \partial_{z} A_{\varepsilon}-\frac{1}{\mathfrak{c}_{s}^{2} \varepsilon^{3}} \partial_{z}\left[f_{4}\left(\varepsilon A_{\varepsilon}\right)\right] \\
& +\frac{1}{\varepsilon}\left\{2 \frac{c(\varepsilon)}{\mathfrak{c}_{s}^{2}} \partial_{z} \varphi_{\varepsilon} \partial_{z} A_{\varepsilon}+\frac{c(\varepsilon)}{\mathfrak{c}_{s}^{2}} A_{\varepsilon} \partial_{z}^{2} \varphi_{\varepsilon}+\frac{1}{\mathfrak{c}_{s}^{2}} \partial_{z}\left[\left(\partial_{z} \varphi_{\varepsilon}\right)^{2}\right]+\left[\frac{1}{2}-\frac{2 r_{0}^{4} f^{\prime \prime}\left(r_{0}^{2}\right)}{\mathfrak{c}_{s}^{2}}\right] \partial_{z}\left(A_{\varepsilon}^{2}\right)\right\}=0 .
\end{aligned}
$$


We must treat carefully the bracket terms, which are formally singular, but the leading order terms cancel out by (15). Using (21) and (22), the bracket term in (23) is

$$
\begin{gathered}
\frac{1}{\varepsilon}\left\{\frac{2}{\mathfrak{c}_{s}} \partial_{z} A_{\varepsilon}\left(\mathfrak{c}_{s} A_{\varepsilon}-\frac{3 \varepsilon}{2} \mathfrak{c}_{s} A_{\varepsilon}^{2}\right)+\frac{1}{\mathfrak{c}_{s}} A_{\varepsilon} \partial_{z}\left(\mathfrak{c}_{s} A_{\varepsilon}-\frac{3 \varepsilon}{2} \mathfrak{c}_{s} A_{\varepsilon}^{2}\right)+\frac{1}{\mathfrak{c}_{s}^{2}} \partial_{z}\left[\left(\mathfrak{c}_{s} A_{\varepsilon}-\frac{3 \varepsilon}{2} \mathfrak{c}_{s} A_{\varepsilon}^{2}\right)^{2}\right]-5 A_{\varepsilon} \partial_{z} A_{\varepsilon}\right\}+\mathcal{O}(\varepsilon) \\
=-15 A_{\varepsilon}^{2} \partial_{z} A_{\varepsilon}+\mathcal{O}(\varepsilon) .
\end{gathered}
$$

As a consequence, passing to the (formal) limit $\varepsilon \rightarrow 0$ in (23) yields

$$
\frac{1}{\mathfrak{c}_{s}^{2}} \partial_{z} A-\frac{1}{\mathfrak{c}_{s}^{2}} \partial_{z}^{3} A+\Gamma^{\prime} A^{2} \partial_{z} A=0, \quad \text { with } \quad \Gamma^{\prime} \equiv-\frac{4 r_{0}^{6} f^{\prime \prime \prime}\left(r_{0}^{2}\right)}{\mathfrak{c}_{s}^{2}}-24 .
$$

This equation is the solitary wave equation for the $(\mathrm{KdV})$ equation with cubic nonlinearity, often called modified Korteweg-de Vries equation $(\mathrm{mKdV})$. The sign of $\Gamma^{\prime}$ plays a fundamental role: if $\Gamma^{\prime}>0$, the underlying $(\mathrm{mKdV})$ equation is defocusing and has no soliton, whereas if $\Gamma^{\prime}<0$, we have a focusing equation with two opposite solitons

$$
\pm \mathrm{w}^{\prime}(z) \equiv \pm \frac{\sqrt{-6 /\left(\Gamma^{\prime} \mathrm{c}_{s}^{2}\right)}}{\cosh (z)}
$$

Indeed, since the nonlinearity is cubic, $A \mapsto-A$ leaves the equation invariant. For this transonic limit, we can prove the following result.

Theorem 5 We assume that, for some $n \in \mathbb{N}, n \geq 4, f \in \mathcal{C}^{n}\left(\mathbb{R}_{+}, \mathbb{R}\right)$ and that $\Gamma=0>\Gamma^{\prime}$. Then, there exists $0<\varepsilon_{0}<\mathfrak{c}_{s}$ such that for every $0<\mathfrak{c}_{0} \equiv c\left(\varepsilon_{0}\right)<c(\varepsilon)<\mathfrak{c}_{s}$, there exist exactly two traveling waves $U_{c(\varepsilon)}^{ \pm}$with speed $c(\varepsilon)$ (up to phase factor and translation). Moreover,

$$
U_{c(\varepsilon)}^{ \pm}(x)=r_{0}\left(1+\varepsilon A_{\varepsilon}^{ \pm}(z)\right) \exp \left(i \varphi_{\varepsilon}^{ \pm}(z)\right), \quad z=\varepsilon x,
$$

with

$$
A_{\varepsilon}^{ \pm} \rightarrow \pm \mathrm{w}^{\prime} \quad \text { and } \quad \partial_{z} \varphi_{\varepsilon}^{ \pm} / \mathfrak{c}_{s} \rightarrow \pm \mathrm{w}^{\prime}
$$

in all spaces $W^{s, p}(\mathbb{R}), s \in \mathbb{N}, 0 \leq s \leq n+2,1 \leq p \leq \infty$. Furthermore, as $\varepsilon \rightarrow 0$,

$$
E\left(U_{c(\varepsilon)}^{ \pm}\right) \sim \mathfrak{c}_{s} P\left(U_{c(\varepsilon)}^{ \pm}\right) \sim-\frac{24 r_{0}^{2}}{\Gamma^{\prime}}\left(\mathfrak{c}_{s}^{2}-c^{2}(\varepsilon)\right)^{\frac{1}{2}}=-\frac{24 r_{0}^{2}}{\Gamma^{\prime}} \varepsilon
$$

and

$$
E\left(U_{c(\varepsilon)}^{ \pm}\right)-c(\varepsilon) P\left(U_{c(\varepsilon)}^{ \pm}\right) \sim-\frac{8 r_{0}^{2}}{\mathfrak{c}_{s}^{2} \Gamma^{\prime}}\left(\mathfrak{c}_{s}^{2}-c^{2}(\varepsilon)\right)^{\frac{3}{2}}=-\frac{8 r_{0}^{2}}{\mathfrak{c}_{s}^{2} \Gamma^{\prime}} \varepsilon^{3} .
$$

Proof. In the case $\Gamma=0$, we recall that we have $2 r_{0}^{4} f^{\prime \prime}\left(r_{0}^{2}\right)=3 \mathfrak{c}_{s}^{2}$, hence

$$
\mathcal{V}(\xi)=\left(\frac{2}{3} f^{\prime \prime}\left(r_{0}^{2}\right)+\frac{1}{6} f^{(3)}\left(r_{0}^{2}\right)\right) \xi^{4}+\mathcal{O}\left(\xi^{5}\right)=-\frac{\Gamma^{\prime} \mathfrak{c}_{s}^{2}}{24 r_{0}^{4}} \xi^{4}+\mathcal{O}\left(\xi^{5}\right),
$$

and thus $\mathcal{V}_{c(\varepsilon)}$ has a graph (for small $\varepsilon$ ) of the following type:

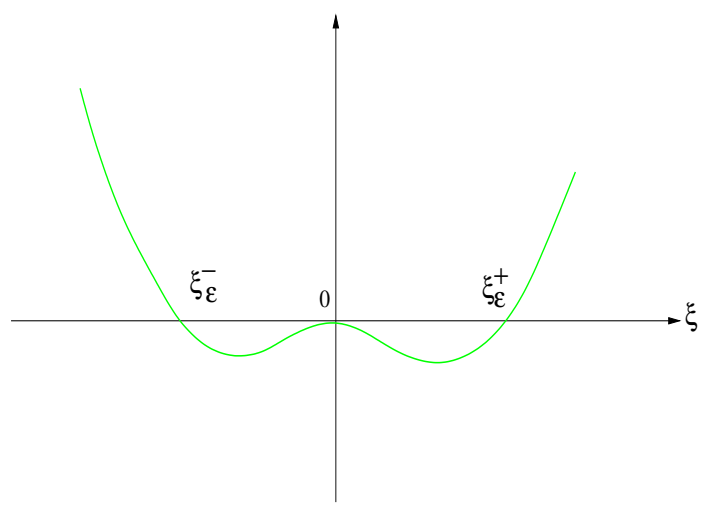

Figure 5: Graph of $\mathcal{V}(\xi)-\varepsilon^{2} \xi^{2}$. 
with two zeros $\xi_{\varepsilon}^{ \pm}= \pm 2 r_{0}^{2} \varepsilon \sqrt{\frac{-6}{\mathfrak{c}_{s}^{2} \Gamma^{\prime}}}+\mathcal{O}\left(\varepsilon^{2}\right)$. Therefore we have two branches of traveling waves and we shall focus on the one corresponding to the interval $\left(0, \xi_{\varepsilon}^{+}\right)$. For the proof of the convergence, we scale

$$
U_{c(\varepsilon)}^{+}(x)=r_{0} \sqrt{1+\varepsilon \mathcal{A}_{\varepsilon}^{+}(z)} \exp \left(i \varphi_{\varepsilon}^{+}(z)\right), \quad z=\varepsilon x,
$$

so that (17) becomes, with $\xi=r_{0}^{2} \varepsilon \zeta$,

$$
z=-\int_{\xi_{\varepsilon}^{+} /\left(r_{0}^{2} \varepsilon\right)}^{\mathcal{A}_{\varepsilon}^{+}(z)} \frac{d \zeta}{\sqrt{\zeta^{2}-\frac{\Gamma^{\prime} \mathfrak{c}_{s}^{2}}{24} \zeta^{4}+\mathcal{O}\left(\varepsilon \zeta^{5}\right)}} .
$$

Passing to the limit as before implies $\mathcal{A}_{\varepsilon}^{+} \rightarrow \mathcal{A}^{+}$uniformly in $\mathbb{R}$, with

$$
z=-\int_{2 \sqrt{-6 /\left(\mathfrak{c}_{s}^{2} \Gamma^{\prime}\right)}}^{\mathcal{A}^{+}(z)} \frac{d \zeta}{\sqrt{\zeta^{2}+\frac{\Gamma^{\prime} \mathfrak{c}_{s}^{2}}{24} \zeta^{4}}},
$$

that is $\mathcal{A}^{+}=2 \mathrm{w}^{\prime}$. The proof of the convergences is then as for Theorem 4 . We now compute as before the asymptotics of the momentum setting $\xi=r_{0}^{2} \varepsilon \zeta$ :

$$
\begin{aligned}
P\left(U_{c(\varepsilon)}^{+}\right) & =c(\varepsilon) \int_{0}^{\xi_{\varepsilon}^{+}} \frac{\xi^{2}}{r_{0}^{2}+\xi} \frac{d \xi}{\sqrt{\varepsilon^{2} \xi^{2}-\mathcal{V}(\xi)}}=r_{0}^{2} \varepsilon c(\varepsilon) \int_{0}^{\xi_{\varepsilon}^{+} /\left(\varepsilon r_{0}^{2}\right)} \frac{\zeta^{2}}{1+\varepsilon \zeta} \frac{d \zeta}{\sqrt{\zeta^{2}+\frac{\Gamma^{\prime} \mathfrak{c}_{s}^{2}}{24} \zeta^{4}+\mathcal{O}\left(\varepsilon \zeta^{5}\right)}} \\
& \sim r_{0}^{2} \varepsilon \mathfrak{c}_{s} \int_{0}^{\sqrt{\frac{-24}{c_{s}^{2} \Gamma^{\prime}}}} \frac{\zeta d \zeta}{\sqrt{1+\frac{\Gamma^{\prime} \mathfrak{c}_{s}^{2}}{24} \zeta^{2}}}=-\varepsilon \frac{24 r_{0}^{2}}{\mathfrak{c}_{s} \Gamma^{\prime}} .
\end{aligned}
$$

Finally,

$$
E\left(U_{c(\varepsilon)}^{+}\right)-c(\varepsilon) P\left(U_{c(\varepsilon)}^{+}\right) \sim r_{0}^{2} \varepsilon^{3} \int_{0}^{\sqrt{\frac{-24}{c_{s}^{2} \Gamma^{\prime}}}} \sqrt{\zeta^{2}+\frac{\Gamma^{\prime} \mathfrak{c}_{s}^{2}}{24} \zeta^{4}} d \zeta=-\frac{8 r_{0}^{2}}{\mathfrak{c}_{s}^{2} \Gamma^{\prime}} \varepsilon^{3} .
$$

The proof is complete.

Of course, we can go further and assume that $\Gamma^{\prime}$ vanishes. If $f$ is sufficiently smooth and

$$
\mathcal{V}(\xi)=\frac{K \xi^{m}}{r_{0}^{2 m-4}}+\mathcal{O}\left(\xi^{m+1}\right) \quad \text { as } \xi \rightarrow 0
$$

with $K \neq 0$ and $m \in \mathbb{N}, m \geq 3$, the natural ansatz will be

$$
U_{c(\varepsilon)}(x)=r_{0}\left(1+\varepsilon^{\frac{2}{m-2}} A_{\varepsilon}(z)\right) \exp \left(i \varepsilon^{\frac{4-m}{m-2}} \varphi_{\varepsilon}(z)\right), \quad z=\varepsilon x .
$$

Indeed, this is for $\xi \simeq \varepsilon^{\frac{2}{m-2}}$ that $\xi^{m} \simeq \varepsilon^{2} \xi^{2}$. We will then have zero, one or two branches of solutions depending on the sign of $K$ and whether $m$ is odd or even. The resulting equation will then be

$$
\frac{1}{\mathfrak{c}_{s}^{2}} \partial_{z} A+\Gamma^{(m)} A^{m-2} \partial_{z} A-\frac{1}{\mathfrak{c}_{s}^{2}} \partial_{z}^{3} A=0
$$

where $\Gamma^{(m)}$ is proportional to $K$, which is the $(\mathrm{gKdV})$ solitary wave equation. If the (gKdV) equation has solitary waves (that is $m$ odd or ( $m$ even and $K>0)$ ), the expansion of the energy and momentum will give, by similar computations,

$$
E\left(U_{c(\varepsilon)}\right)=E_{0} \varepsilon^{\frac{6-m}{m-2}}+\mathcal{O}\left(\varepsilon^{\frac{8-m}{m-2}}\right) \quad \text { and } \quad P\left(U_{c(\varepsilon)}\right)=P_{0} \varepsilon^{\frac{6-m}{m-2}}+\mathcal{O}\left(\varepsilon^{\frac{8-m}{m-2}}\right),
$$


with $E_{0}=\mathfrak{c}_{s} P_{0}$, and we shall have

$$
E\left(U_{c(\varepsilon)}\right)-c(\varepsilon) P\left(U_{c(\varepsilon)}\right)=\operatorname{sgn}\left(\xi_{\varepsilon}\right) \int_{0}^{\xi_{\varepsilon}} \frac{\sqrt{\varepsilon^{2} \xi^{2}+K \xi^{m} / r_{0}^{2 m-4}+\mathcal{O}\left(\xi^{m+1}\right)}}{r_{0}^{2}+\xi} d \xi \sim r_{0}^{2} \varepsilon^{\frac{m+2}{m-2}} S\left(K, \mathfrak{c}_{s}\right),
$$

where $S\left(K, \mathfrak{c}_{s}\right)$ is an integral depending only on $K$ and $\mathfrak{c}_{s}$. Notice that the smallest integer $m$ for which energy and momentum diverge to $+\infty$ as $\varepsilon \rightarrow 0$ is $m=7$. Moreover, we see that

$$
E\left(U_{c(\varepsilon)}\right)-\mathfrak{c}_{s} P\left(U_{c(\varepsilon)}\right)=E\left(U_{c(\varepsilon)}\right)-c(\varepsilon) P\left(U_{c(\varepsilon)}\right)-\left(\mathfrak{c}_{s}-c(\varepsilon)\right) P\left(U_{c(\varepsilon)}\right)=\mathcal{O}\left(\varepsilon^{\frac{m+2}{m-2}}\right)+\mathcal{O}\left(\varepsilon^{2}\right) \times \mathcal{O}\left(\varepsilon^{\frac{6-m}{m-2}}\right)=\mathcal{O}\left(\varepsilon^{\frac{m+2}{m-2}}\right)
$$

tends to zero as $\varepsilon \rightarrow 0$, hence the straight line $E=\mathfrak{c}_{s} P$ is always an asymptote for $m \geq 7$ (with $P\left(U_{c(\varepsilon)}\right) \gg 1$ and $E\left(U_{c(\varepsilon)}\right) \gg 1$ for $\left.\varepsilon \ll 1\right)$.

Concerning the stability of the solitary wave in the transonic limit, we would like to mention some stability results of the $(\mathrm{gKdV})$ soliton (with speed $\left.\frac{1}{\mathfrak{c}_{s}^{2}}\right)$ in the $(\mathrm{gKdV})$ equation

$$
\partial_{\tau} A-\frac{1}{\mathfrak{c}_{s}^{2}} \partial_{z}^{3} A+\Gamma^{(m)} A^{m-2} \partial_{z} A=0
$$

when this soliton exists, that is $m$ odd or $m$ even and $\Gamma^{(m)}<0$. It is known to be stable in the subcritical case, i.e. for $m \leq 5$, (see [9]) and unstable if $m \geq 6$ ( $c f$. [9] for the supercritical case $m \geq 7$ and [26] for the critical case $m=6)$. Furthermore, in the critical case $(m=6)$, blow-up in finite time do occur for some initial data close to the (gKdV) soliton ([27]). Notice that for the critical case $m=6$ (and $\left.\Gamma^{(6)}<0\right), E\left(U_{c(\varepsilon)}\right)$ and $P\left(U_{c(\varepsilon)}\right)$ converge to some finite positive limits as $\varepsilon \rightarrow 0$, with $\lim _{\varepsilon \rightarrow 0} E\left(U_{c(\varepsilon)}\right)=\mathfrak{c}_{s} \lim _{\varepsilon \rightarrow 0} P\left(U_{c(\varepsilon)}\right)>0$ (since $E\left(U_{c(\varepsilon)}\right)-\mathfrak{c}_{s} P\left(U_{c(\varepsilon)}\right)$ always tends to 0 ). This means that in the (focusing) critical case $m=6$, the curve $c \mapsto(E, P)$ has a stopping point located on the straight line $E=\mathfrak{c}_{s} P$.

\section{$3 \quad$ Study of some model cases}

In this section, we consider some particular but relevant nonlinearities and for each of them we find all non constant traveling waves to (NLS) satisfying the condition $|U(x)| \rightarrow r_{0}$ as $x \rightarrow \pm \infty$. We shall always have $r_{0}=1$. To be consistent with the $(\mathrm{KdV})$ limit, we always relate the speed $c$ to $\varepsilon$ by

$$
\mathfrak{c}_{s}^{2}=c^{2}+\varepsilon^{2}
$$

but $\varepsilon$ needs not to be small. Our starting point is the case $\varepsilon=0$ (that is $c=\mathfrak{c}_{s}$ ). We then let $\varepsilon \in\left[0, \mathfrak{c}_{s}\right]$ increase from 0 to $\mathfrak{c}_{s}$. For some values of $\varepsilon$ (or $c$ ), we draw the potential

$$
[-1,+\infty) \ni \xi \mapsto \mathcal{V}_{c}(\xi) \equiv \mathcal{V}(\xi)-\varepsilon^{2} \xi^{2}=\mathcal{V}(\xi)-\left(\mathfrak{c}_{s}^{2}-c^{2}\right) \xi^{2}
$$

in (9), from which it is easy to see if there exists or not a solution to (8) with $|U| \rightarrow r_{0}$ at infinity. For the selected representative values of $\varepsilon$ (or $c$ ), we represent the qualitative behaviour of the squared modulus $|U|^{2}=r_{0}^{2}+\eta$ of the solution (there is no need to integrate numerically the ODE, since the global shape of $|U|^{2}$ follows immediately from the graph of $\mathcal{V}_{c}$ ). For the diagrams of energy/momentum/speed, we have computed numerically the energy and momentum using the formulae in subsection 1.2. It may happen that for some particular values of $\varepsilon$ (or $c$ ) the integrals tend to infinity rather slowly, and then it is difficult to capture this divergence numerically.

The choices of each nonlinearity has been done in order to illustrate in particular the different behaviours described through the transonic limit, hence we construct some $f^{\prime}$ 's with $\Gamma=0$ and both signs of $\Gamma^{\prime}$, and very degenerate situations corresponding to a transonic limit governed by some (gKdV) solitary wave equation. The first three model cases are based on a polynomial nonlinearity $f$ of degree three, thus we call them cubic-quintic-septic nonlinearities. 


\subsection{Example 1: a cubic-quintic-septic nonlinearity (I)}

We consider the nonlinearity

$$
f(\varrho) \equiv-(\varrho-1)+\frac{3}{2}(\varrho-1)^{2}-\frac{3}{2}(\varrho-1)^{3} .
$$

Then, we compute

$$
V(\varrho)=\frac{1}{2}(\varrho-1)^{2}-\frac{1}{2}(\varrho-1)^{3}+\frac{3}{8}(\varrho-1)^{4} \quad \text { and } \quad \mathcal{V}(\xi)=\frac{\xi^{4}}{2}-\frac{3 \xi^{5}}{2},
$$

so that $r_{0}=1, \mathfrak{c}_{s}^{2}=2, \Gamma=0$ and $\Gamma^{\prime}=-6$, and the graphs of $f, V$ and $\mathcal{V}$ are

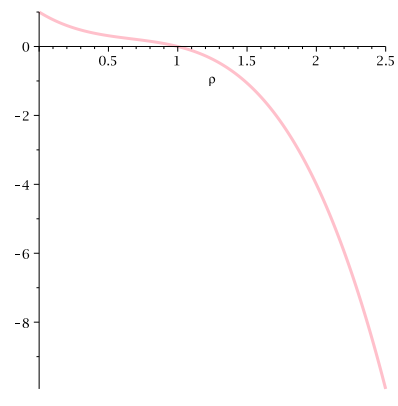

(a)

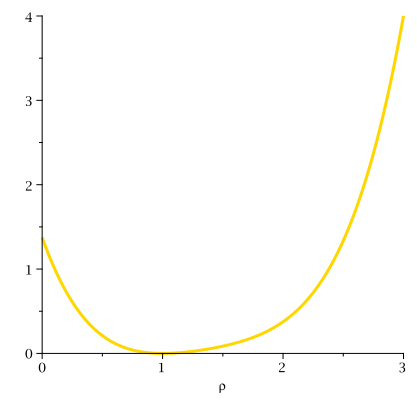

(b)

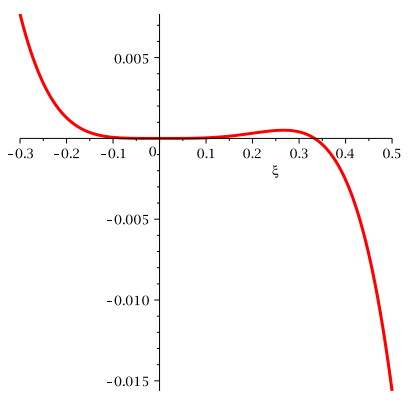

(c)

Figure 6: Graphs of (a) $f$, (b) $V$ and (c) $\mathcal{V}$

This model case has been chosen in order to illustrate a case where $\Gamma=0$ and $\Gamma^{\prime}<0$, for which, according to Theorem 5, we have two branches of solutions in the transonic limit. Actually, for $\varepsilon$ small, we have

$$
\mathcal{V}_{c}(\xi)=\frac{\xi^{4}}{2}-\frac{3 \xi^{5}}{2}-\varepsilon^{2} \xi^{2}
$$

and this function, for $\varepsilon$ small enough, has two zeros

$$
\xi_{\varepsilon}^{+}=\sqrt{2} \varepsilon+\mathcal{O}\left(\varepsilon^{2}\right) \quad \xi_{\varepsilon}^{-}=-\sqrt{2} \varepsilon+\mathcal{O}\left(\varepsilon^{2}\right)
$$

near the origin, with $\mathcal{V}_{c}(\xi)<0$ in $\left(\xi_{\varepsilon}^{-}, 0\right)$ and in $\left(0, \xi_{\varepsilon}^{+}\right)$. It follows that there exist two traveling waves with speed $c$, one with $|U|>r_{0}$ (for the $\left(0, \xi_{\varepsilon}^{+}\right)$part, called the upper solution), and one with $|U|<r_{0}$ (for the $\left(\xi_{\varepsilon}^{-}, 0\right)$ part, called the lower solution). 


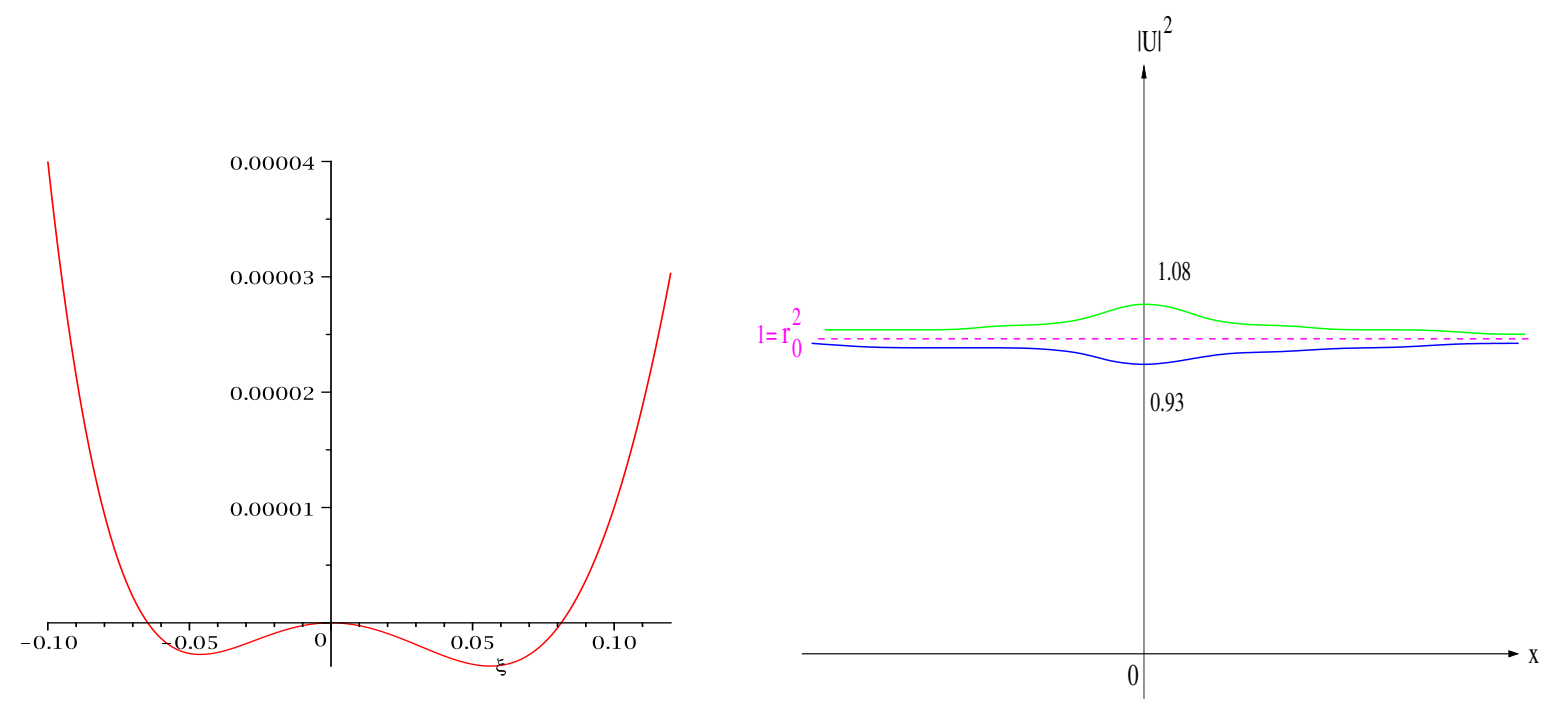

(a)

(b)

Figure 7: Graphs of (a) $\mathcal{V}_{c}$ and (b) $|U|^{2}$ for $c=\sqrt{2-0.05^{2}} \simeq 1.4133<\mathfrak{c}_{s}=\sqrt{2} \simeq 1.4142$

We then increase $\varepsilon$.

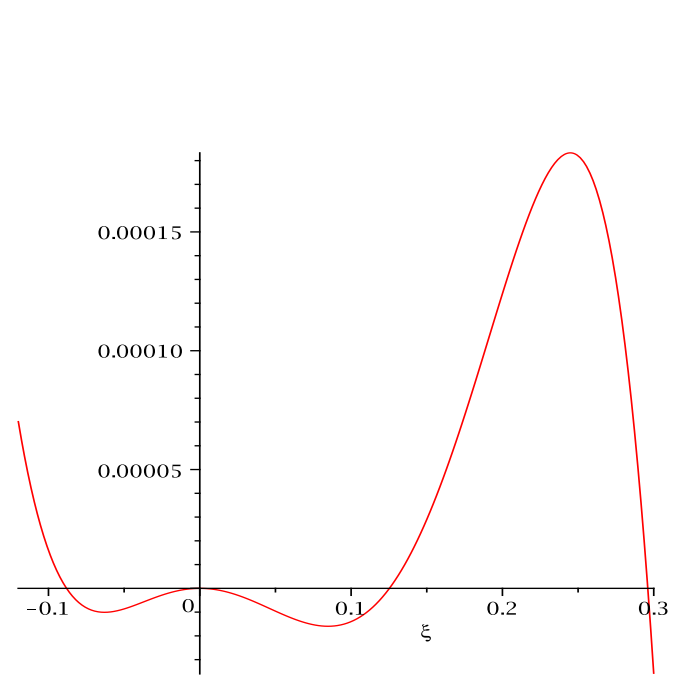

(a)

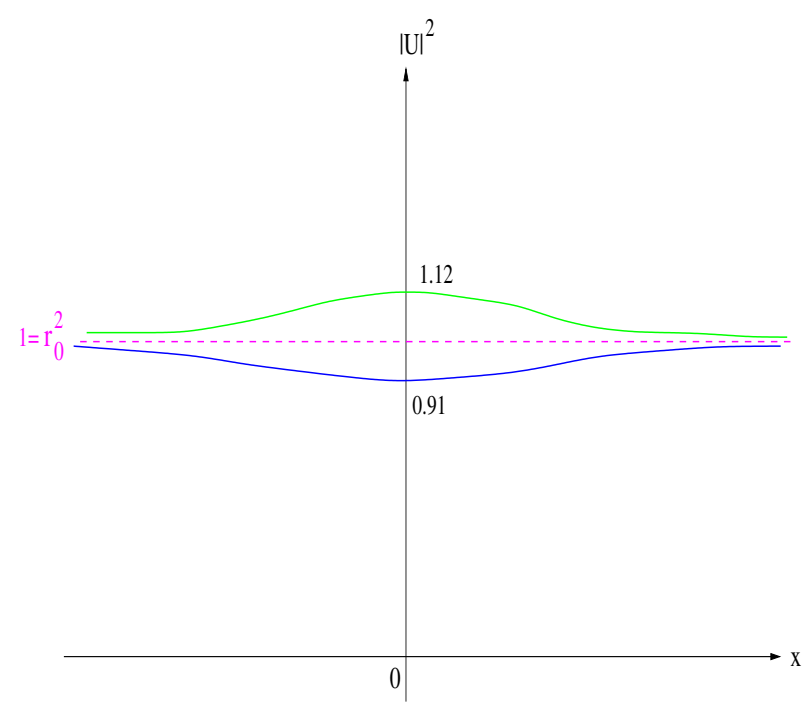

(b)

Figure 8: Graphs of (a) $\mathcal{V}_{c}$ and (b) $|U|^{2}$ for $c=\sqrt{2-0.07^{2}} \simeq 1.4124<\mathfrak{c}_{s}=\sqrt{2} \simeq 1.4142$ 


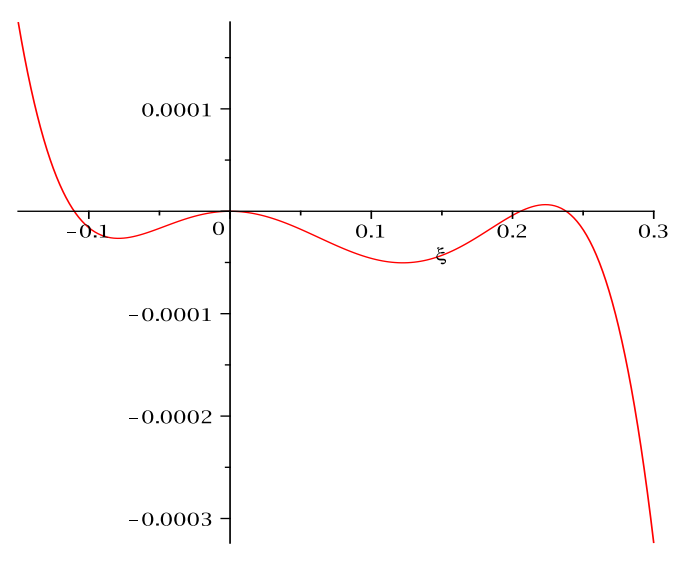

(a)

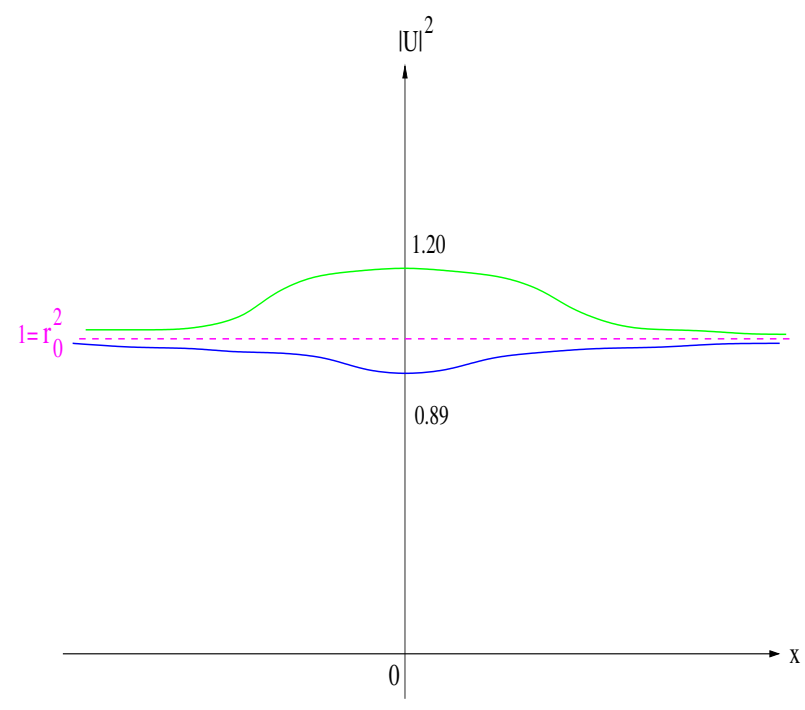

(b)

Figure 9: Graphs of (a) $\mathcal{V}_{c}$ and (b) $|U|^{2}$ for $c=\sqrt{2-0.09^{2}} \simeq 1.1411347<\mathfrak{c}_{s}=\sqrt{2} \simeq 1.4142$

We observe that if we now increase slightly $\varepsilon$, the potential $\mathcal{V}_{c}$ will have a double positive root that can be computed explicitely. Indeed, one has

$$
2 \mathcal{V}_{c}(\xi)=\xi^{4}-3 \xi^{5}-2 \varepsilon^{2} \xi^{2}=\xi^{2}\left(\xi^{2}-3 \xi^{3}-2 \varepsilon^{2}\right),
$$

and the discriminant of the cubic polynomial in parenthesis vanishes only for $\varepsilon=\varepsilon_{0} \equiv \frac{2}{9 \sqrt{6}} \simeq 0.0907$. The double positive root is then $\frac{2}{9} \simeq 0.222$, and this corresponds to the critical speed $c_{0}=\sqrt{2-\varepsilon_{0}^{2}} \simeq 1.4113007$. For $0<\varepsilon<\varepsilon_{0}$, the potential $\mathcal{V}_{c}$ remains negative on two intervals $\left(\xi_{\varepsilon}^{-}, 0\right)$ and $\left(0, \xi_{\varepsilon}^{+}\right)$, and there exist exactly two solutions to $\left(\mathrm{TW}_{c}\right)$. As $\varepsilon \rightarrow \varepsilon_{0}^{-}$, the squared modulus of the upper solution in figure 9 tends to the constant $r_{0}^{2}+\frac{2}{9} \simeq 1.222$ locally in space. From (6) (with $K=0$ ), it follows that, locally in space,

$$
\phi^{\prime} \rightarrow \frac{\sqrt{2-\varepsilon_{0}^{2}}}{2} \frac{2 / 9}{1+2 / 9}=\frac{1}{11} \sqrt{\frac{484}{243}} .
$$

In particular, as $\varepsilon \rightarrow \varepsilon_{0}^{-}$, the traveling wave associated to the upper solution converges, locally in space, to

$$
\frac{11}{9} \exp \left(\frac{i x}{11} \sqrt{\frac{484}{243}}\right)
$$

(up to a phase factor), which is a nontrivial solution of $\left(\mathrm{TW}_{c_{0}}\right)$ but not a traveling wave (it does not have neither modulus one at infinity nor finite energy). We recall that the invariance by translation is frozen by imposing that $|U|^{2}$ is even. It is straightforward to show that for this solution, both energy and momentum diverge to $+\infty$. For our nonlinearity, it turns out that for $\varepsilon \geq \varepsilon_{0}$, we do not have any nontrivial traveling wave with modulus $>r_{0}$, that is associated to the part $\xi>0$ for $\mathcal{V}_{c}$. However, the solution in the part $\xi<0$ for $\mathcal{V}_{c}$ remains. 


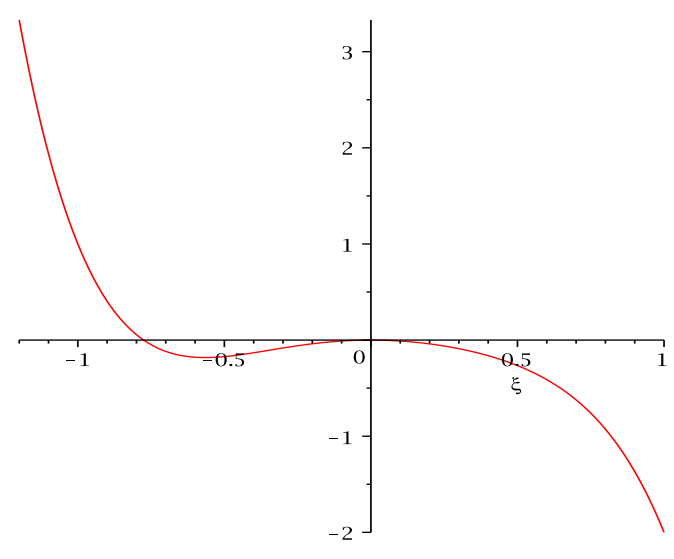

(a)

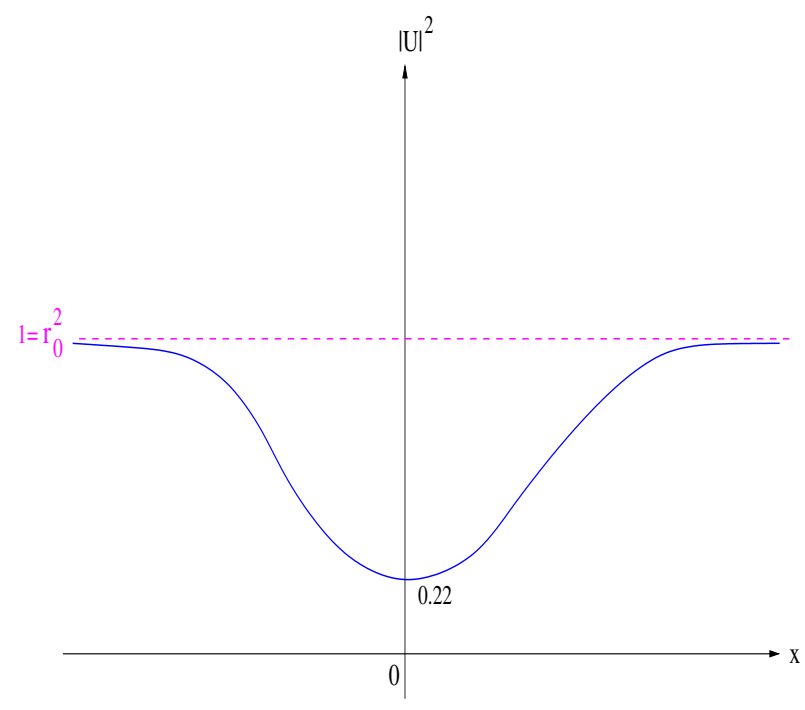

(b)

Figure 10: Graphs of (a) $\mathcal{V}_{c}$ and (b) $|U|^{2}$ for $c=\sqrt{2-1^{2}}=1<\mathfrak{c}_{s}=\sqrt{2} \simeq 1.4142$

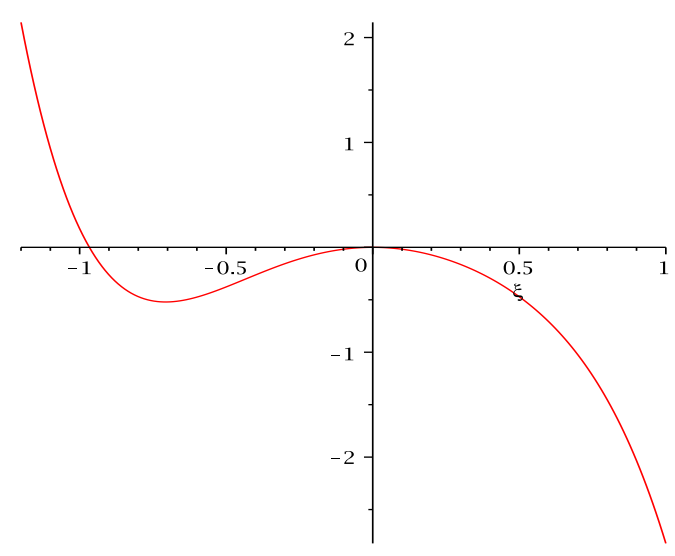

(a)

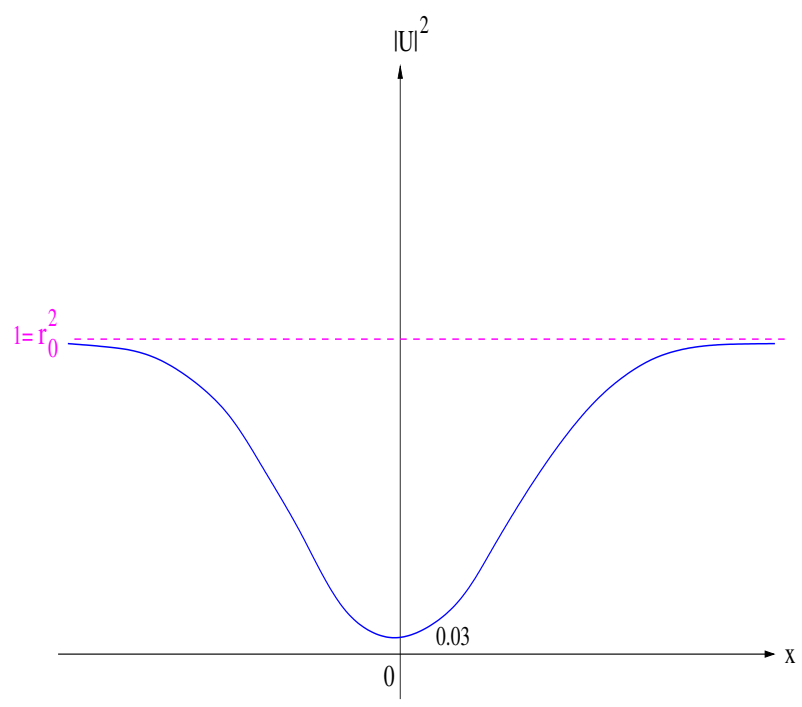

(b)

Figure 11: Graphs of (a) $\mathcal{V}_{c}$ and (b) $|U|^{2}$ for $c=\sqrt{2-1.35^{2}} \simeq 0.421<\mathfrak{c}_{s}=\sqrt{2} \simeq 1.4142$

Using the numerical values we have obtained, we may now plot for the two branches of traveling waves the energy and momentum with respect to the speed, as well as the energy-momentum diagram. 


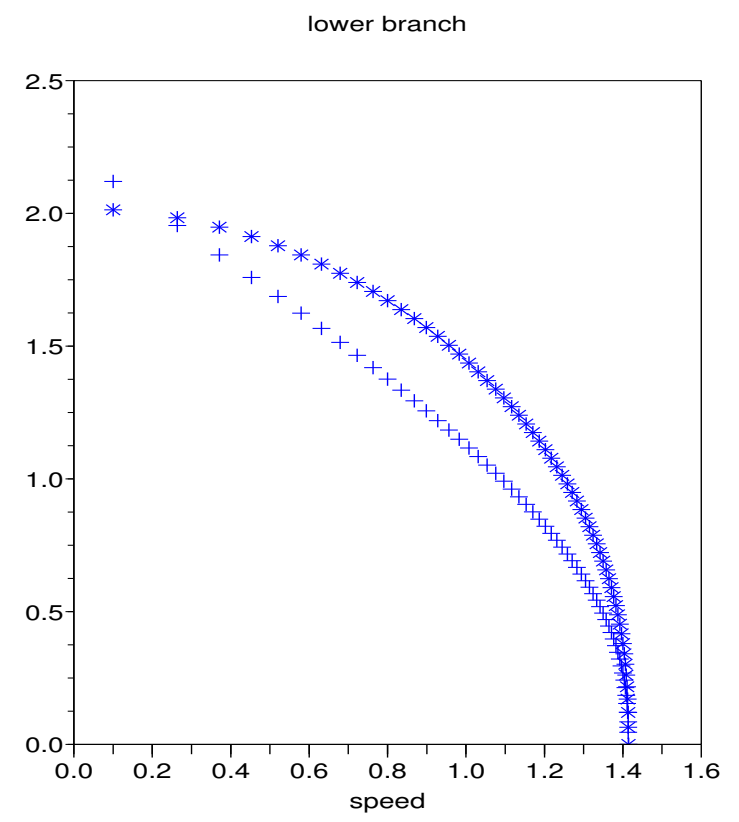

(a)

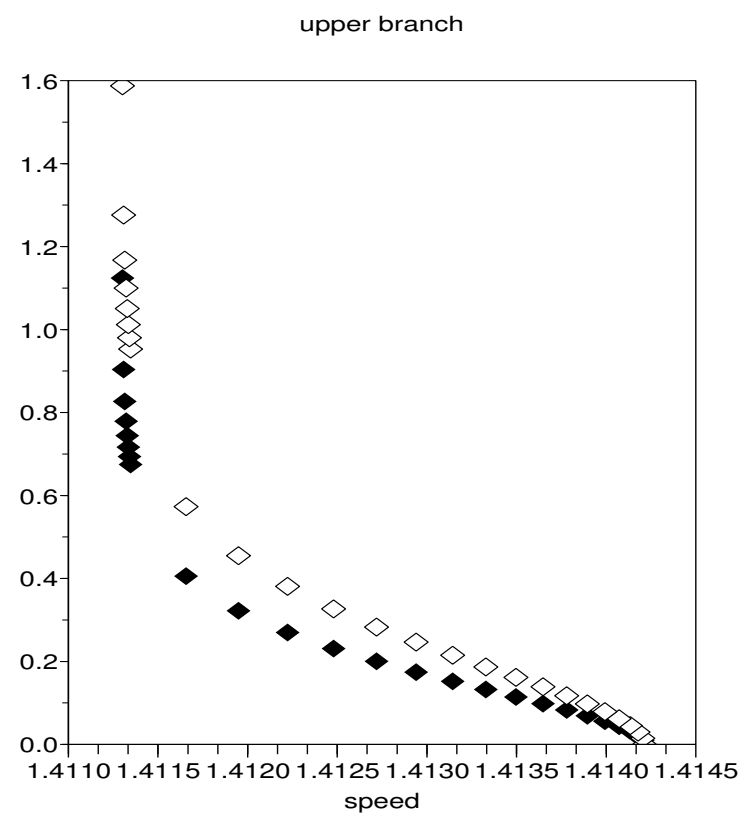

(b)

Figure 12: (a) Energy $\left(^{*}\right)$ and momentum $(+)$ for the lower branch; (b) Energy $(\diamond)$ and momentum $(\diamond)$ for the upper branch.

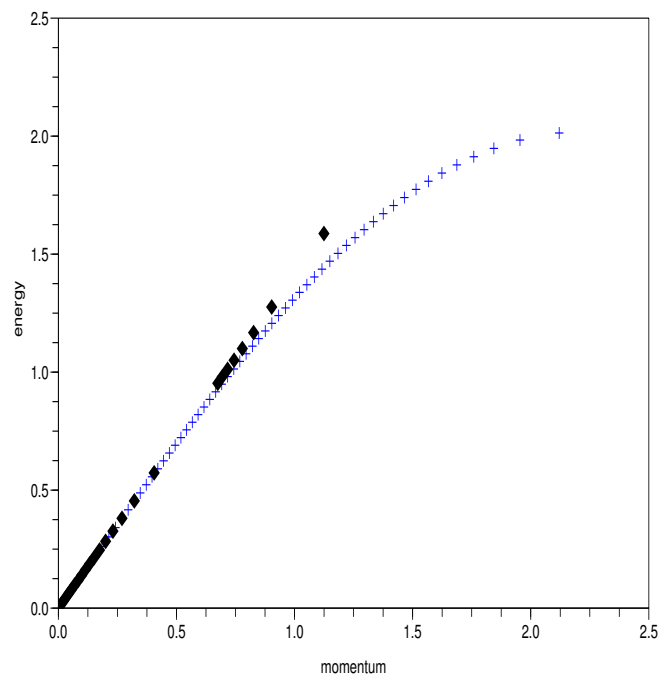

Figure 13: The numerical $(E, P)$ diagram

As $\varepsilon \rightarrow \varepsilon_{0}^{-}$and for the upper solution, both energy and momentum diverge to $+\infty$. From the expressions 
in subsection 1.2 , it follows that

$$
P=-\frac{E_{0}}{c_{0}} \ln \left(\frac{c}{c_{0}}-1\right)+P_{1}+\mathcal{O}\left(c-c_{0}\right) \quad \text { and } \quad E=-E_{0} \ln \left(\frac{c}{c_{0}}-1\right)+E_{1}+\mathcal{O}\left(c-c_{0}\right)
$$

as $c \rightarrow c_{0}^{+}$, for some constants $E_{0}>0, E_{1}$ and $P_{1}$. This divergence is therefore not easy to capture numerically. Note however that this shows that we have an asymptote $E-E_{1}=c_{0}\left(P-P_{1}\right)$. The graphs of $E$ and $P$ with respect to the speed $c$ and the full $(E, P)$ diagram (where the straight dashed line is $E=\mathfrak{c}_{s} P$ ) for this example have the following shape.

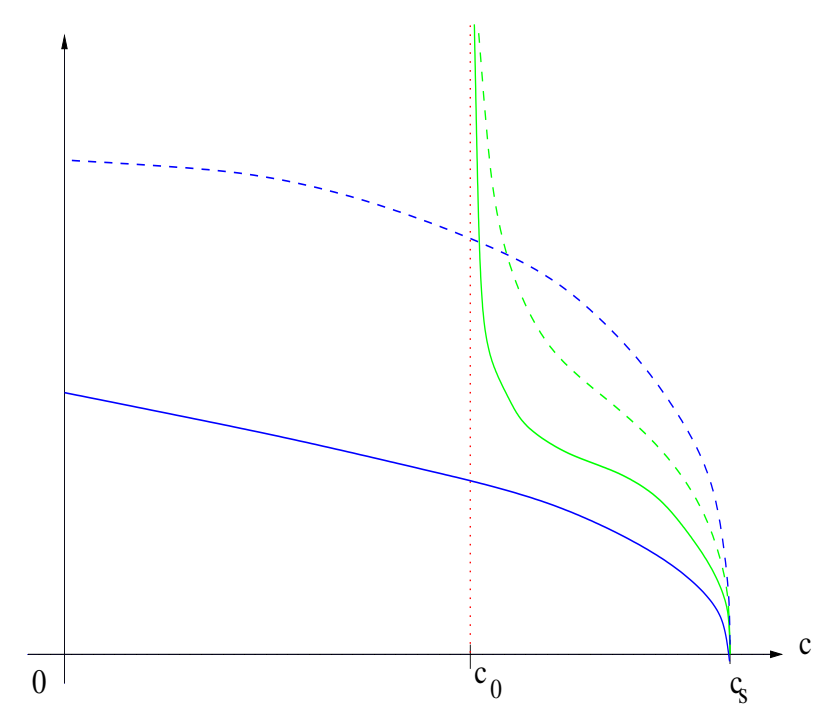

(a)

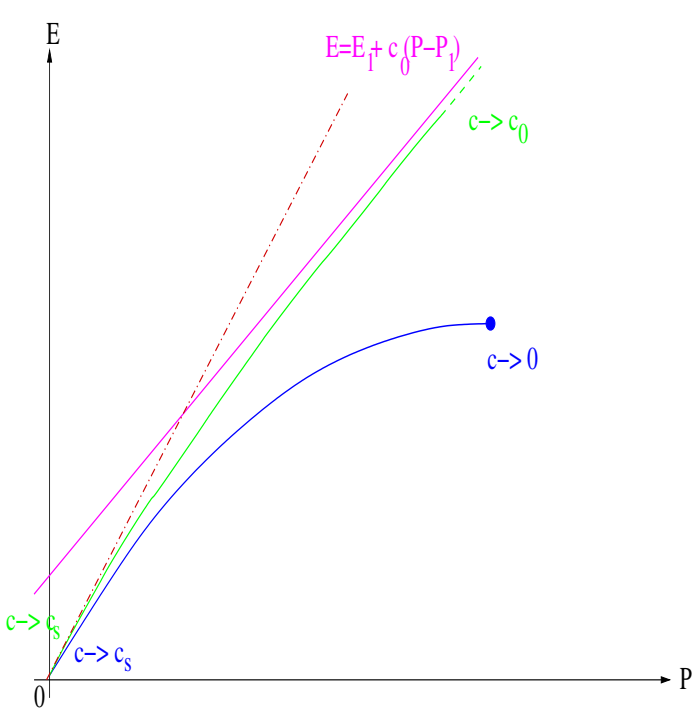

(b)

Figure 14: (a) Energy (dashed curve) and momentum (full curve) vs. speed; (b) (E, P) diagram

Comments. Let us point out some qualitative facts that we can observe from this example. We have constructed the nonlinearity $f$ so that the potential $V$ has the same qualitative behaviour as the one associated to the Gross-Pitaevskii nonlinearity ( $f$ is decreasing, tends to $-\infty$ at $+\infty$ ). However, due to the cancellation of the coefficient $\Gamma$, which is a second order condition on $f$ at $\varrho=r_{0}^{2}$, we have two solutions in the transonic limit $c \rightarrow \mathfrak{c}_{s}$. To our knowledge, this is the first multiplicity result of this type. On the other hand, there exist solutions with high energy and momentum. Furthermore, we see that it may happen that a familly of traveling waves solutions to $\left(\mathrm{TW}_{c}\right)$ with modulus tending to $r_{0}$ at infinity converges as $c \rightarrow c_{0} \in\left(0, \mathfrak{c}_{s}\right)$ to a nontrivial solution to $\left(\mathrm{TW}_{c_{0}}\right)$ which does not have modulus $r_{0}$ at infinity. Therefore, for a general smooth decreasing nonlinearity $f$, this example shows that it is not true that the (exponential) decay of the traveling waves at infinity can be made uniform for speeds in a compact interval $\left[c_{\min }, c_{\max }\right] \subset\left(0, \mathfrak{c}_{s}\right)$.

\subsection{Example 2: a cubic-quintic-septic nonlinearity (II)}

Here, we consider

$$
f(\varrho) \equiv-4(\varrho-1)-36(\varrho-1)^{3}
$$

We compute

$$
V(\varrho)=2(\varrho-1)^{2}+9(\varrho-1)^{4} \quad \text { and } \quad \mathcal{V}(\xi)=-8 \xi^{3}-36 \xi^{4}-36 \xi^{5}
$$

thus $r_{0}=1, \mathfrak{c}_{s}^{2}=8, \Gamma=6$, and the graphs of $f, V$ and $\mathcal{V}$ are 


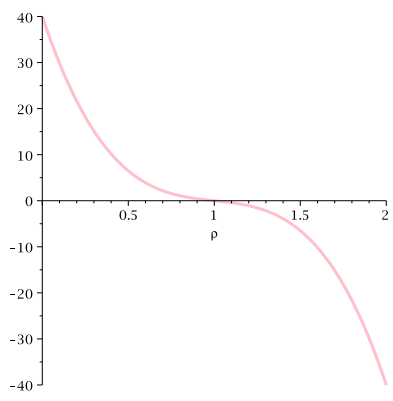

(a)

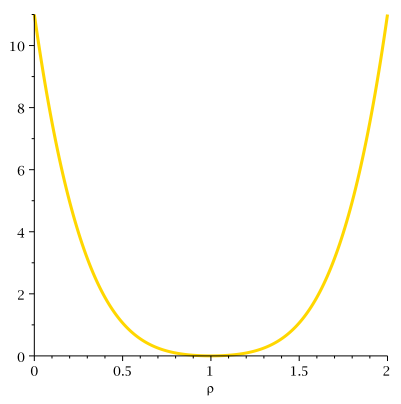

(b)

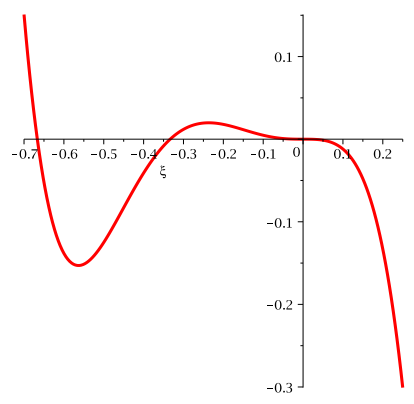

(c)

Figure 15: Graphs of (a) $f$, (b) $V$ and (c) $\mathcal{V}$

Compared to example 1, this time the potential $\mathcal{V}_{c}$ will have, for some $0<c<\mathfrak{c}_{s}$, a double root located in the part $\xi<0$. Observe that $\mathcal{V}(-0.25)>0$. Here again, we let $\varepsilon \in\left[0, \mathfrak{c}_{s}\right]$ increase.

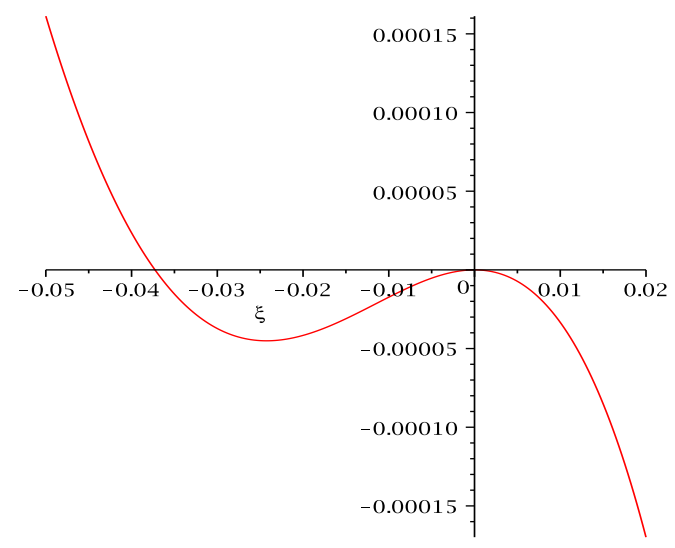

(a)

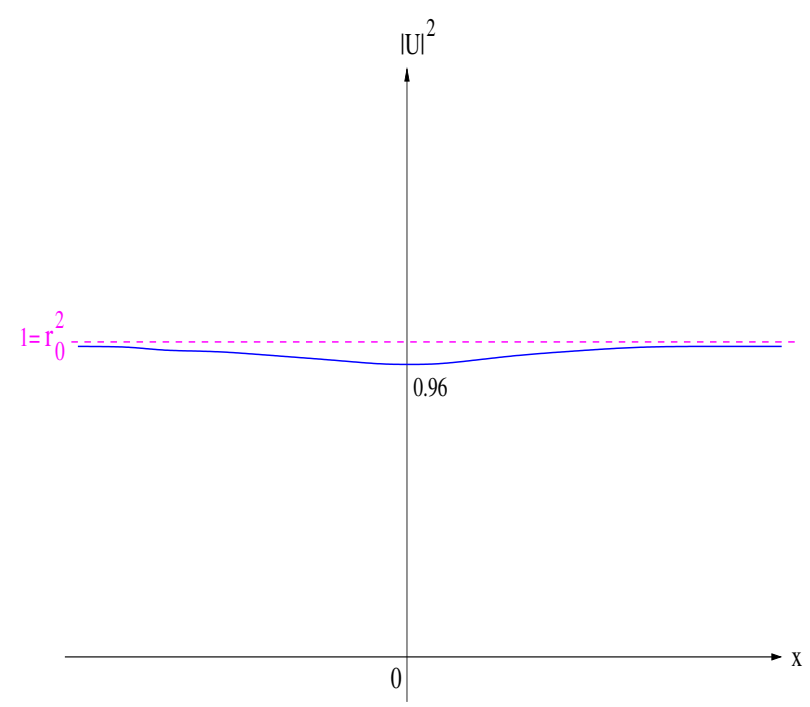

(b)

Figure 16: Graphs of (a) $\mathcal{V}_{c}$ and (b) $|U|^{2}$ for $c=\sqrt{8-0.5^{2}} \simeq 2.7839<\mathfrak{c}_{s}=\sqrt{8} \simeq 2.8284$ 


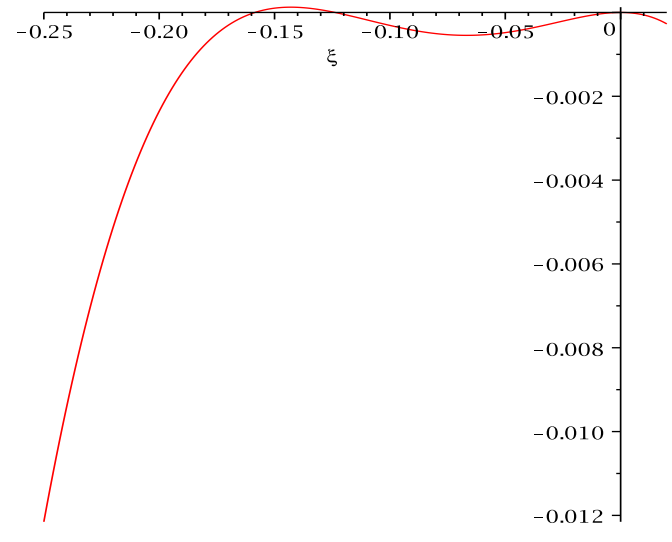

(a)

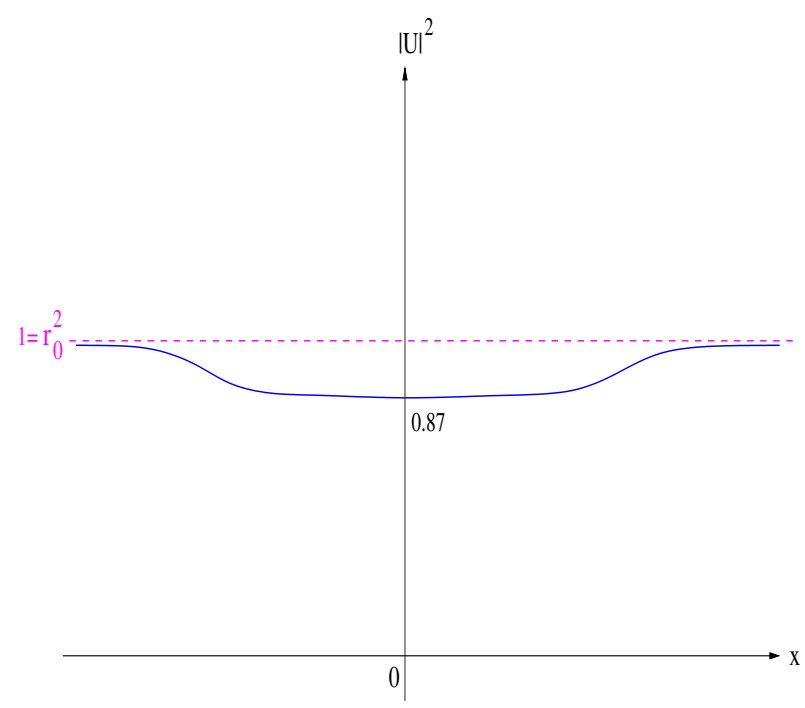

(b)

Figure 17: Graphs of (a) $\mathcal{V}_{c}$ and (b) $|U|^{2}$ for $c=\sqrt{8-0.712^{2}} \simeq 2.7373<\mathfrak{c}_{s}=\sqrt{8} \simeq 2.8284$

Here, we find a situation similar to Example 1. If we slightly increase $\varepsilon$, the potential $\mathcal{V}_{c}$ will have a double root. Here again, we may compute explicitely the critical value $\varepsilon_{0}$ noticing that we have

$$
\mathcal{V}_{c}(\xi)=-36 \xi^{2}\left(\xi^{3}+\xi^{2}+2 \xi / 9+\varepsilon^{2} / 36\right),
$$

and it is easily checked that the discriminant of the cubic polynomial in parenthesis is $\left(\varepsilon^{2} / 16-4 / 3^{5}\right) / 9^{2}$ and thus vanishes only for $\varepsilon=\varepsilon_{0} \equiv 2 \sqrt[4]{4 / 3^{5}} \simeq 0.7163$, which corresponds to the critical speed $c_{0}=\sqrt{8-\varepsilon_{0}^{2}} \simeq$ 2.7362. The negative double root is then $\simeq-0.1409$. In figure 17 , we are just before this critical value, and this forces $\eta=|U|^{2}-r_{0}^{2}\left(\right.$ or $|U|^{2}$ ) to stay on a rather long range of $x$ close to the value $\simeq-0.14$ (or 0.86 ). As for example 1 , as $\varepsilon \rightarrow \varepsilon_{0}^{-}$, the traveling wave solution converges, locally in space, to a function of the type

$$
\alpha \exp (i \beta x),
$$

for some constants $\alpha \simeq 0.9269$ and $\beta \simeq-0.2244$. 


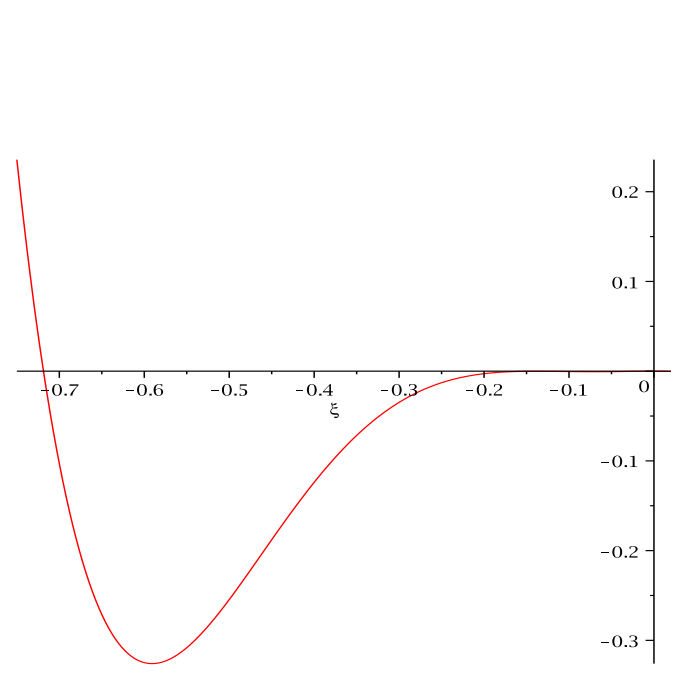

(a)

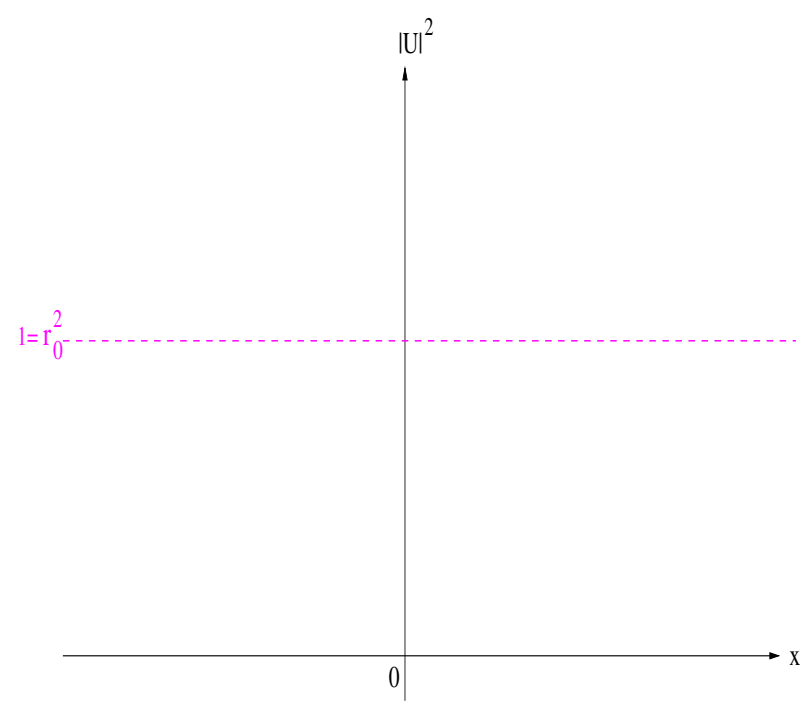

(b)

Figure 18: Graphs of (a) $\mathcal{V}_{c}$ and (b) $|U|^{2}$ for $c=c_{0} \equiv \sqrt{8-\varepsilon_{0}^{2}} \simeq 2.7362<\mathfrak{c}_{s}=\sqrt{8} \simeq 2.8284$

In figure 18 , we are exactly on the critical value $\varepsilon=\varepsilon_{0}=2 \sqrt[4]{4 / 3^{5}} \simeq 0.7163$. The potential $\mathcal{V}_{c_{0}}$ is negative between 0 and the negative root $\simeq-0.1409$, but this root is a double root: no traveling wave exists for this critical value $c=c_{0}$.

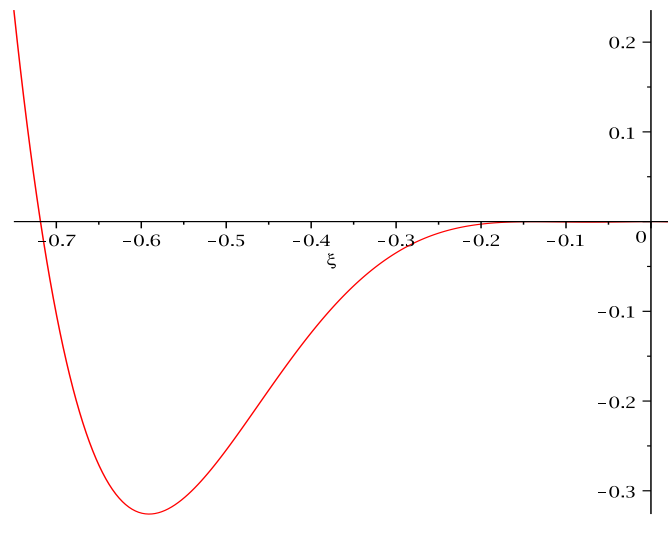

(a)

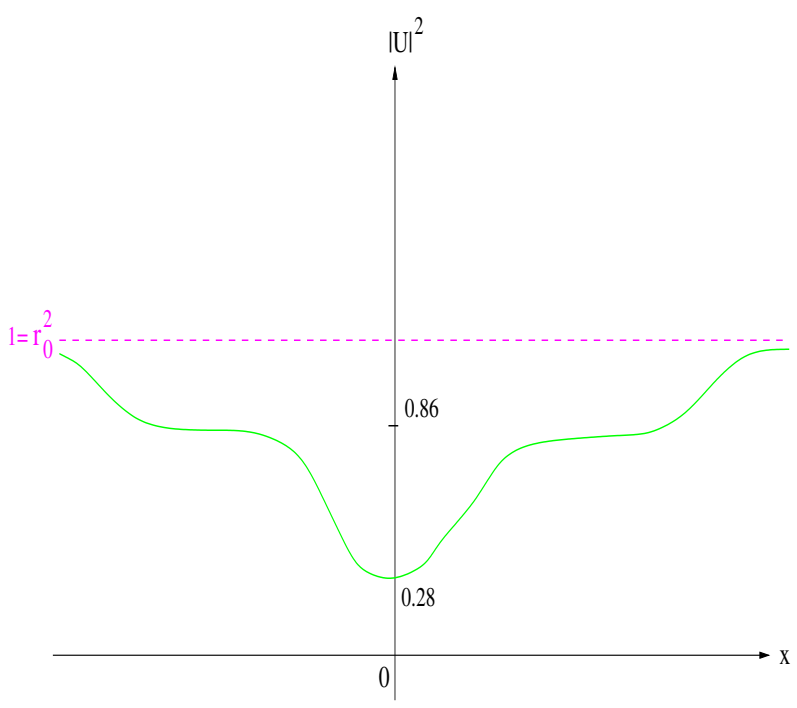

(b)

Figure 19: Graphs of (a) $\mathcal{V}_{c}$ and (b) $|U|^{2}$ for $c=\sqrt{8-0.72^{2}} \simeq 2.735<\mathfrak{c}_{s}=\sqrt{8} \simeq 2.8284$

For figure 19 , we have $\varepsilon$ slightly greater than $\varepsilon_{0}$ : the potential $\mathcal{V}_{c}$ is then negative between 0 and $\simeq-0.7187$, but has a local maximum at $\simeq-0.14$, which is very close to zero, namely $\simeq-0.000102$. The minimum value of $\eta=|U|^{2}-r_{0}^{2}$ (or $|U|^{2}$ ) is then $\simeq-0.7187$ (or $\simeq 0.28$ ), but $\eta$ (or $|U|^{2}$ ) remains close to $\simeq-0.14$ (or $\simeq 0.86$ ) for two quite large $x$ intervals. If we decrease $\varepsilon$ to $\varepsilon_{0}$, we see that $\eta$ converges, locally in space, to the homoclinic solution (which remains between $\simeq-0.1409$ and $\simeq-0.7187$ ) associated to the double negative root $\simeq-0.1409$ of $\mathcal{V}_{c_{0}}$. In figure 15 (c), it can be noticed that there exists a solution to $\left(\mathrm{TW}_{\mathfrak{c}_{s}}\right)$ (but not a traveling wave) with periodic modulus corresponding to the region $\xi \in(-2 / 3,-1 / 3)$ in 
which $\mathcal{V}$ is $<0$, with $\mathcal{V}(-2 / 3)=0>\mathcal{V}^{\prime}(-2 / 3)$ and $\mathcal{V}(-1 / 3)=0<\mathcal{V}^{\prime}(-1 / 3)$. As $\varepsilon$ increases up to $\varepsilon_{0}$, the period increases to infinity. For $\varepsilon=1.5$, the picture is the following.

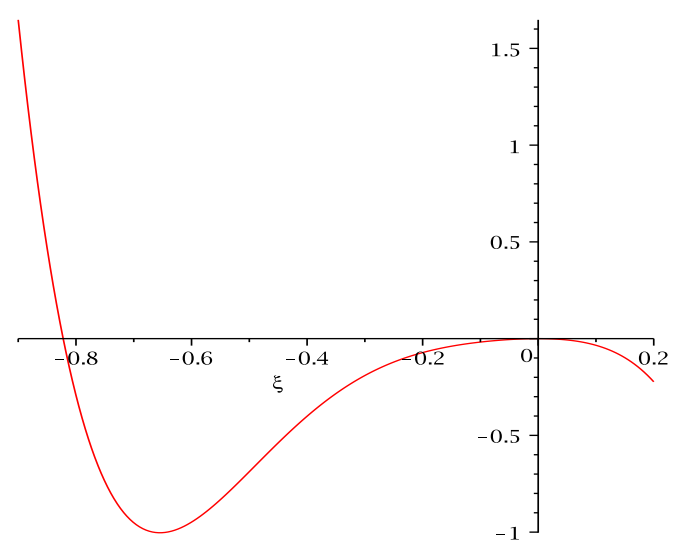

(a)

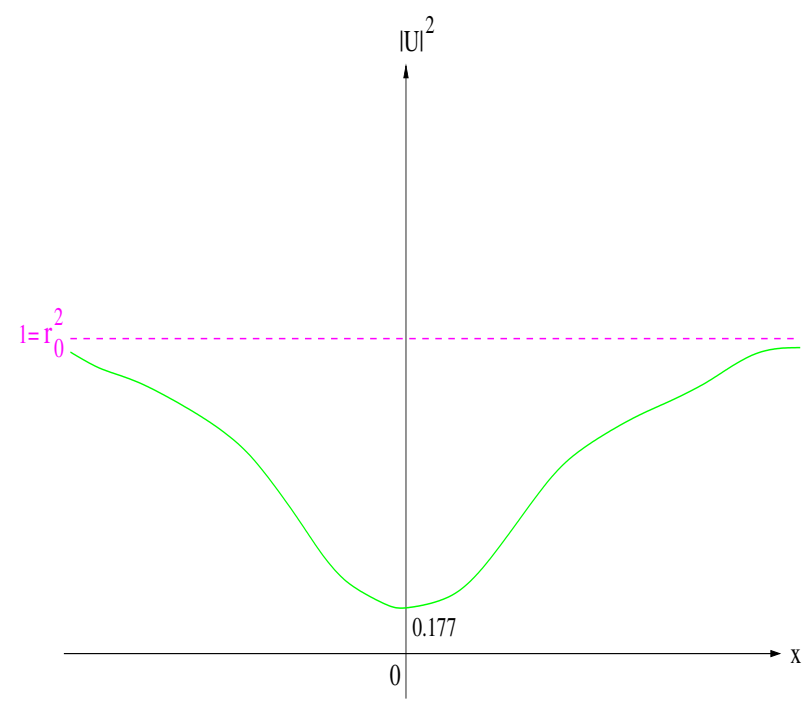

(b)

Figure 20: Graphs of (a) $\mathcal{V}_{c}$ and (b) $|U|^{2}$ for $c=\sqrt{8-1.5^{2}} \simeq 2.3979<\mathfrak{c}_{s}=\sqrt{8} \simeq 2.8284$

We now show the graphs of energy and momentum for the two branches of solutions (for the numerical values we have obtained) with respect to the speed: $c_{0}<c<\mathfrak{c}_{s}$ for the first branch, $0 \leq c<c_{0}$ for the second one, as well as the $(E, P)$ diagram.

first branch

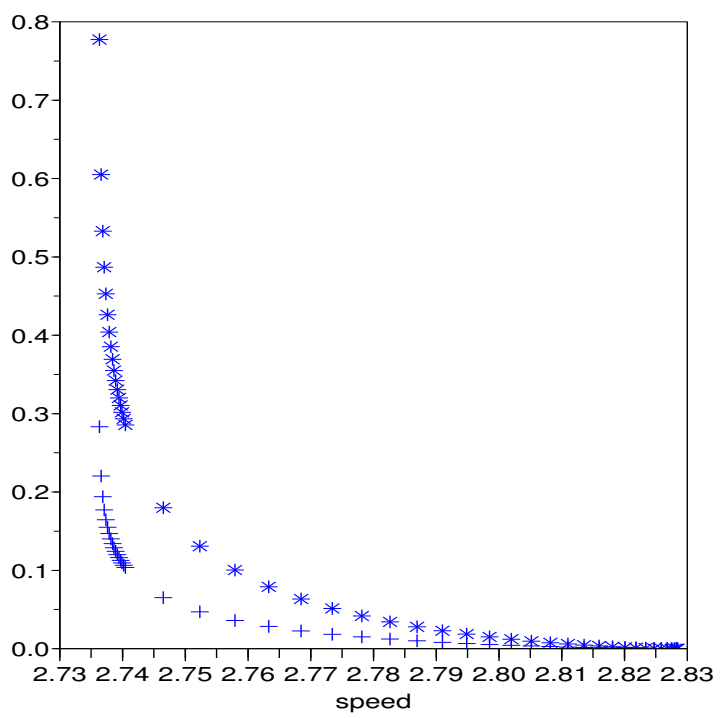

(a)

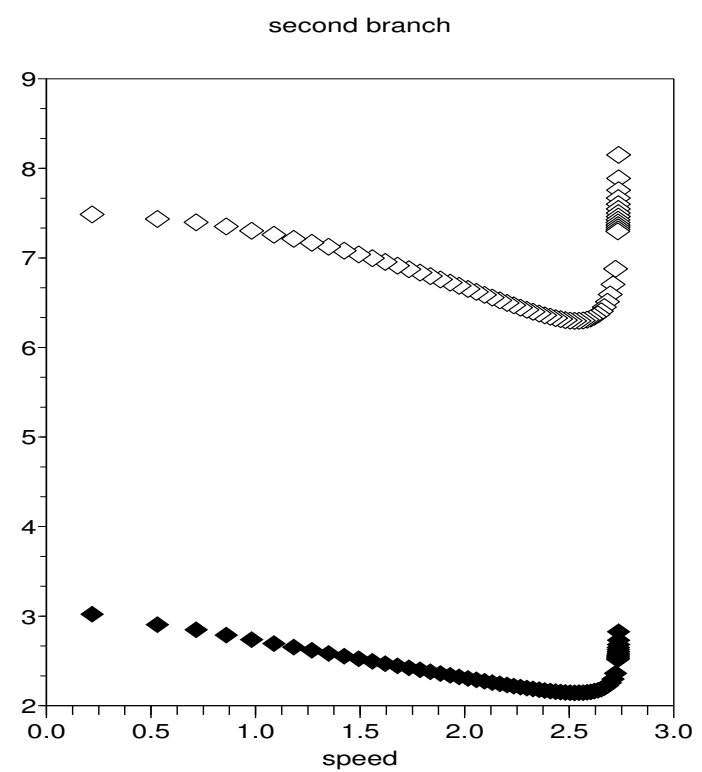

(b)

Figure 21: (a) Energy $\left(^{*}\right)$ and momentum $(+)$ for the first branch; (b) Energy $(\diamond)$ and momentum $(\diamond)$ for the second branch. 


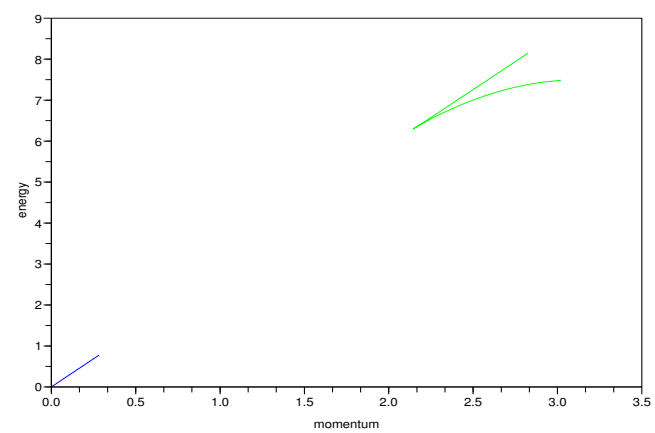

Figure 22: Energy (dashed curve) and momentum (full curve)

We observe a cusp for some speed $c_{\text {cusp }} \simeq 2.55$. This corresponds to the common minimum of both energy and momentum in figure 21 (b).

Concerning the behaviour when $c \rightarrow c_{0}$, we have expansions similar to those for example 1 :

$$
\begin{aligned}
& P=-\frac{E_{0}^{+}}{c_{0}} \ln \left(\frac{c}{c_{0}}-1\right)+P_{1}^{+}+\mathcal{O}\left(c-c_{0}\right), \quad E=-E_{0}^{+} \ln \left(\frac{c}{c_{0}}-1\right)+E_{1}^{+}+\mathcal{O}\left(c-c_{0}\right) \quad \text { as } \quad c \rightarrow c_{0}^{+} \\
& P=-\frac{E_{0}^{-}}{c_{0}} \ln \left(1-\frac{c}{c_{0}}\right)+P_{1}^{-}+\mathcal{O}\left(c_{0}-c\right) \quad E=-E_{0}^{-} \ln \left(1-\frac{c}{c_{0}}\right)+E_{1}^{-}+\mathcal{O}\left(c_{0}-c\right) \quad \text { as } \quad c \rightarrow c_{0}^{-},
\end{aligned}
$$

for some constants $E_{0}^{ \pm}>0, E_{1}^{ \pm}$and $P_{1}^{ \pm}$. Notice that this means that the divergence of the energy and the momentum for the first branch is extremely slow, hence very difficult to capture numerically. The constants $E_{0}^{ \pm}>0$ verify $E_{0}^{-}=2 E_{0}^{+}$. Moreover, from the expressions of the energy and momentum in subsection 1.2, it comes

$$
\begin{aligned}
E\left(U_{c}\right)-c_{0} P\left(U_{c}\right) & =4 \operatorname{sgn}\left(\xi_{c}\right) \int_{0}^{\xi_{c}} \frac{V\left(r_{0}^{2}+\xi\right)}{\sqrt{-\mathcal{V}_{c}(\xi)}} d \xi-c c_{0} \operatorname{sgn}\left(\xi_{c}\right) \int_{0}^{\xi_{c}} \frac{\xi^{2}}{(1+\xi) \sqrt{-\mathcal{V}_{c}(\xi)}} d \xi \\
& =c\left(c-c_{0}\right) \operatorname{sgn}\left(\xi_{c}\right) \int_{0}^{\xi_{c}} \frac{\xi^{2}}{(1+\xi) \sqrt{-\mathcal{V}_{c}(\xi)}} d \xi+\operatorname{sgn}\left(\xi_{c}\right) \int_{0}^{\xi_{c}} \frac{\sqrt{-\mathcal{V}_{c}(\xi)}}{1+\xi} d \xi,
\end{aligned}
$$

where we use the expression of $\mathcal{V}_{c}$ in the before last equality. The first integral diverges as $\mathcal{O}\left(\ln \left|\frac{c}{c_{0}}-1\right|\right)$ as $c \rightarrow c_{0}^{ \pm}$, hence the first term has a contribution $\mathcal{O}\left(\left|c-c_{0}\right| \ln \left|\frac{c}{c_{0}}-1\right|\right) \ll 1$. Therefore, as $c \rightarrow c_{0}$,

$$
E\left(U_{c}\right)-c_{0} P\left(U_{c}\right)=\operatorname{sgn}\left(\xi_{c}\right) \int_{0}^{\xi_{c}} \frac{\sqrt{-\mathcal{V}_{c}(\xi)}}{1+\xi} d \xi+\mathcal{O}\left(\left|c-c_{0}\right| \ln \left|\frac{c}{c_{0}}-1\right|\right) \rightarrow \operatorname{sgn}\left(\xi_{c_{0}}\right) \int_{0}^{\xi_{c_{0}}} \frac{\sqrt{-\mathcal{V}_{c_{0}}(\xi)}}{1+\xi} d \xi .
$$

This means that we have an asymptote for $c \rightarrow c_{0}^{ \pm}$

$$
E=c_{0} P+E_{0}, \quad E_{0} \equiv \operatorname{sgn}\left(\xi_{c_{0}}\right) \int_{0}^{\xi_{c_{0}}} \frac{\sqrt{-\mathcal{V}_{c_{0}}(\xi)}}{1+\xi} d \xi>0
$$

(hence the constants $E_{1}^{ \pm}$and $P_{1}^{ \pm}$verify $E_{1}^{ \pm}-c_{0} P_{1}^{ \pm}=E_{0}$ ).

The full $(E, P)$ diagram (here again, the straight dashed line is $E=\mathfrak{c}_{s} P$ ) has thus the following shape. It is difficult to check numerically that the two curves actually cross, since the divergence is extremely slow. However, for the first branch, the curve $P \mapsto E$ is concave. Indeed, we have seen in subsection 2.1 that $\frac{d E}{d P}=c$ and $c$ decreases along this branch. Moreover, a numerical integration gives $E_{0} \simeq 0.0512$. Since $E=E_{0}+c_{0} P$ 
is an asymptote, by concavity, all points $(P, E)$ on the first branch verify $c_{0} P \leq E \leq E_{0}+c_{0} P$. In particular, for $P=P\left(U_{c=0}\right) \simeq 3.127$, we deduce that the solution of the first branch has an energy $\geq c_{0} \times 3.127 \simeq 8.5557$. However, the solution for $c=0$ has an energy $7.5023<8.5557$. Thus, for the same momentum $\simeq 3.127$, the solution for $c=0$ is strictly below the corresponding solution for $c \simeq c_{0}$ on the first branch.

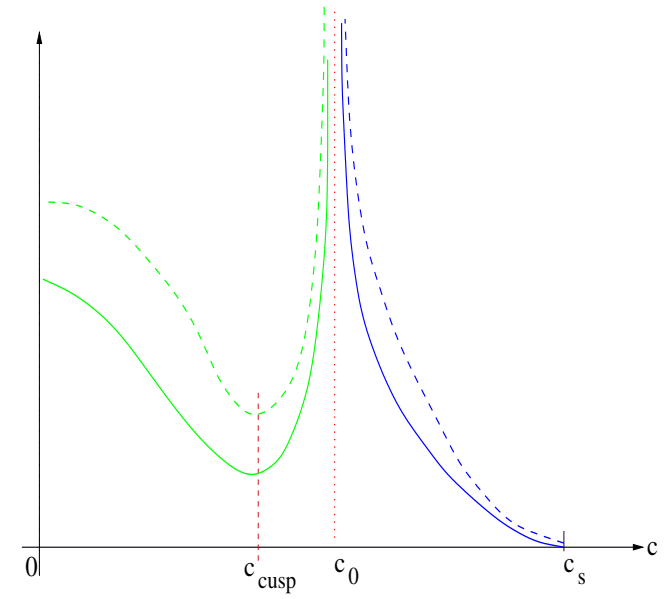

(a)

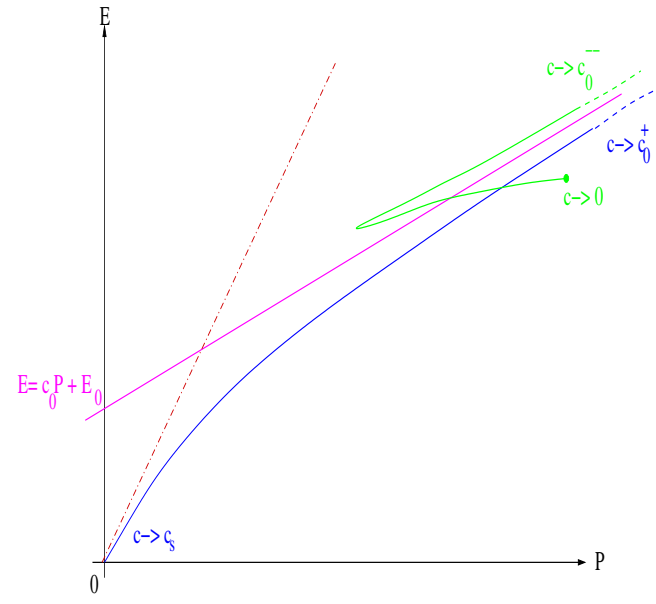

(b)

Figure 23: (a) Energy (dashed curve) and momentum (full curve) vs. speed; (b) $(E, P)$ diagram

Comments. This example shows that, contrary to what is usually expected, the set of speeds for which there exist nontrivial traveling waves may be different from $\left[0, \mathfrak{c}_{s}\right)$ (here this set is $\left[0, c_{0}\right) \cup\left(c_{0}, \mathfrak{c}_{s}\right)$ for some $\left.0<c_{0}<\mathfrak{c}_{s}\right)$. Furthermore, we observe a cusp as it is the case for the three-dimensional traveling waves for the Gross-Pitaevskii equation (see [19]). To our knowledge, this is the first mathematical evidence of such a cusp for a nonlinearity $f$ such that the potential $V$ is nonnegative (see however the case of the cubic-quintic

nonlinearity in subsection 3.6). Let us emphasize that Theorem 3 does not apply when $\frac{d P}{d c}=0$, which is what happens at the cusp. Therefore, the stability of the solution associated to the cusp is not known. Finally, we observe two branches of solutions that cross at some point, which, to our knowledge, has never been observed.

\subsection{Example 3: a cubic-quintic-septic nonlinearity (III)}

We consider here

$$
f(\varrho) \equiv-\frac{1}{2}(\varrho-1)+\frac{3}{4}(\varrho-1)^{2}-2(\varrho-1)^{3},
$$

for which

$$
V(\varrho)=\frac{1}{4}(\varrho-1)^{2}-\frac{1}{4}(\varrho-1)^{3}+\frac{1}{2}(\varrho-1)^{4} \quad \text { and } \quad \mathcal{V}(\xi)=-\xi^{4}-2 \xi^{5},
$$

thus $r_{0}=1, \mathfrak{c}_{s}^{2}=1, \Gamma=0, \Gamma^{\prime}=24>0$, and the graphs of $f, V$ and $\mathcal{V}$ are 


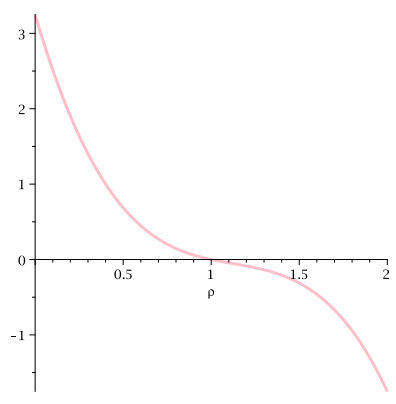

(a)

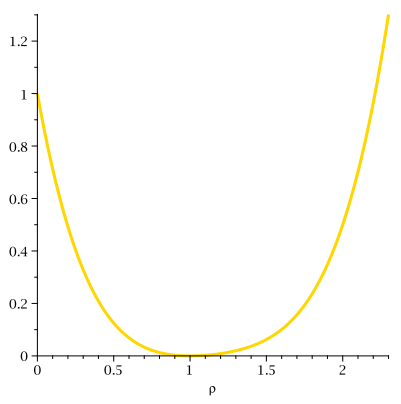

(b)

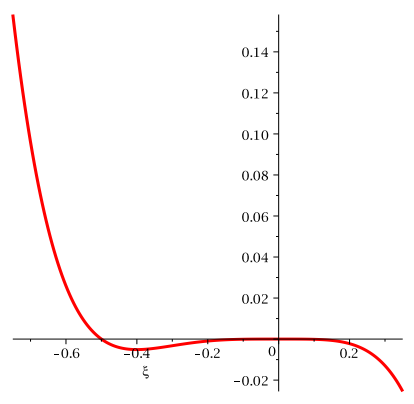

(c)

Figure 24: Graphs of (a) $f$, (b) $V$ and (c) $\mathcal{V}$

This nonlinearity was chosen in order to illustrate the case $\Gamma=0$ and $\Gamma^{\prime}>0$, in which case the modified Korteweg-de Vries equation $(\mathrm{mKdV})$ is defocusing and has no solitary wave. Here again, we let $\varepsilon \in\left[0, \mathfrak{c}_{s}\right]$ increase from 0 to $\mathfrak{c}_{s}=1$, but start with $\varepsilon=0$.

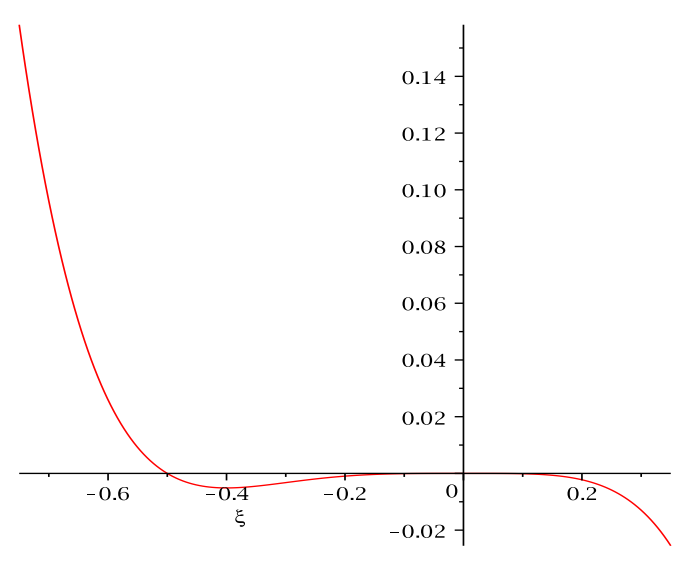

(a)

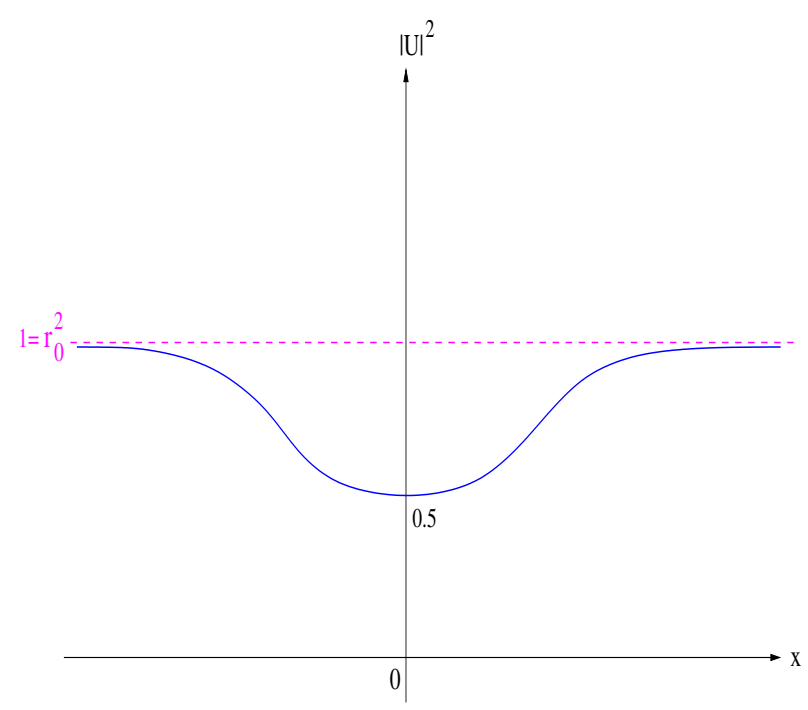

(b)

Figure 25: Graphs of (a) $\mathcal{V}_{c}$ and (b) $|U|^{2}$ for $c=\mathfrak{c}_{s}=1$

For this nonlinearity $f$, it turns out that there exists exactly one (up to translations in space and the multiplication by a phase factor) sonic nonconstant traveling wave (figure 25). In particular, in the transonic limit, the traveling waves converge to this particular solution, which has nonzero energy $(=1.6)$ and momentum $(=\pi / 2)$. 


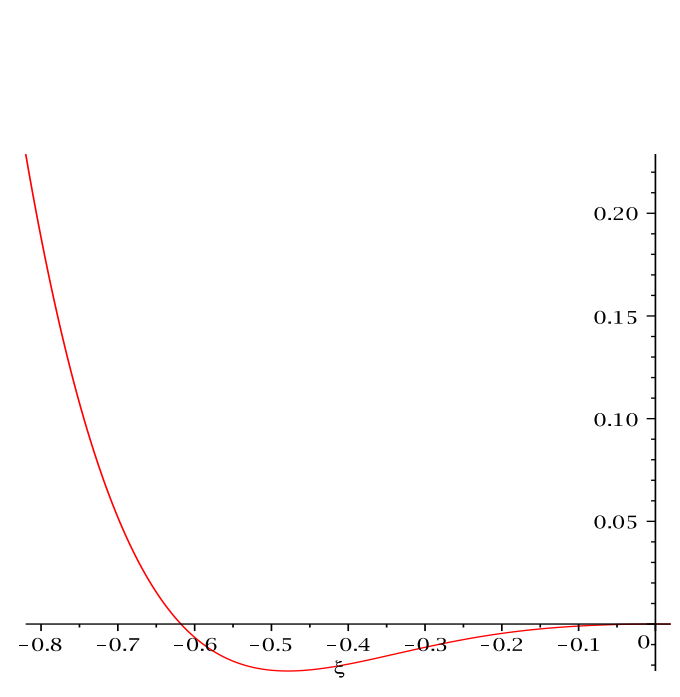

(a)

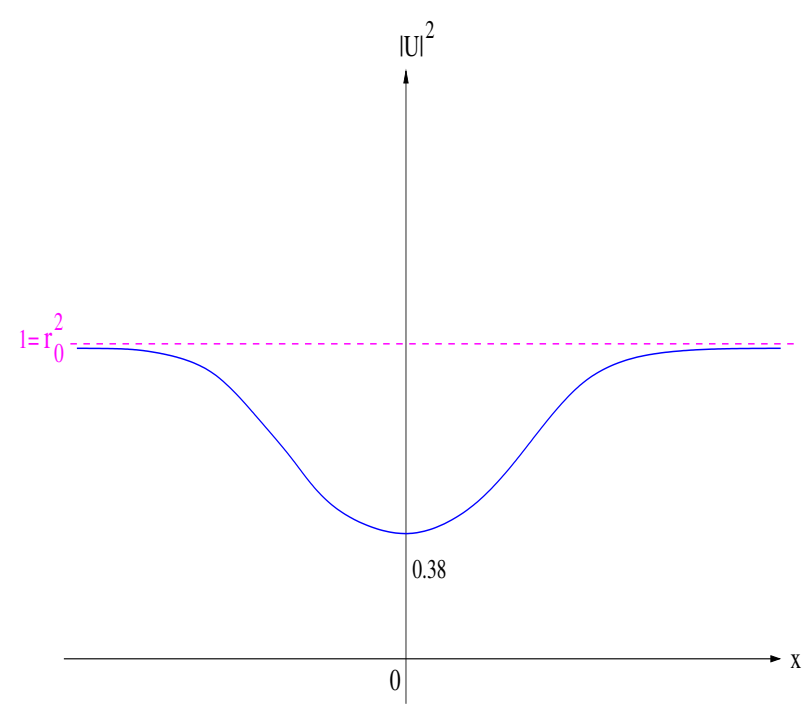

(b)

Figure 26: Graphs of (a) $\mathcal{V}_{c}$ (red) (b) $|U|^{2}$ for $c=\sqrt{1-0.3^{2}} \simeq 0.9539<\mathfrak{c}_{s}=1$

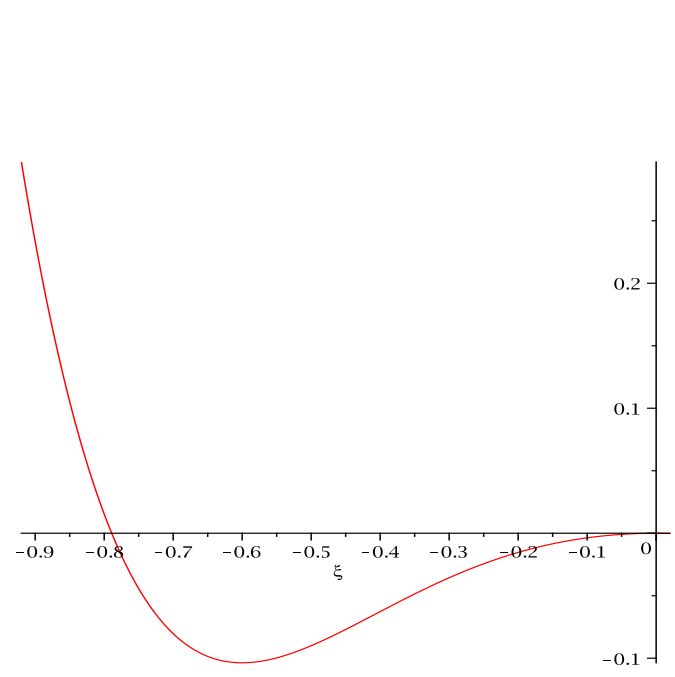

(a)

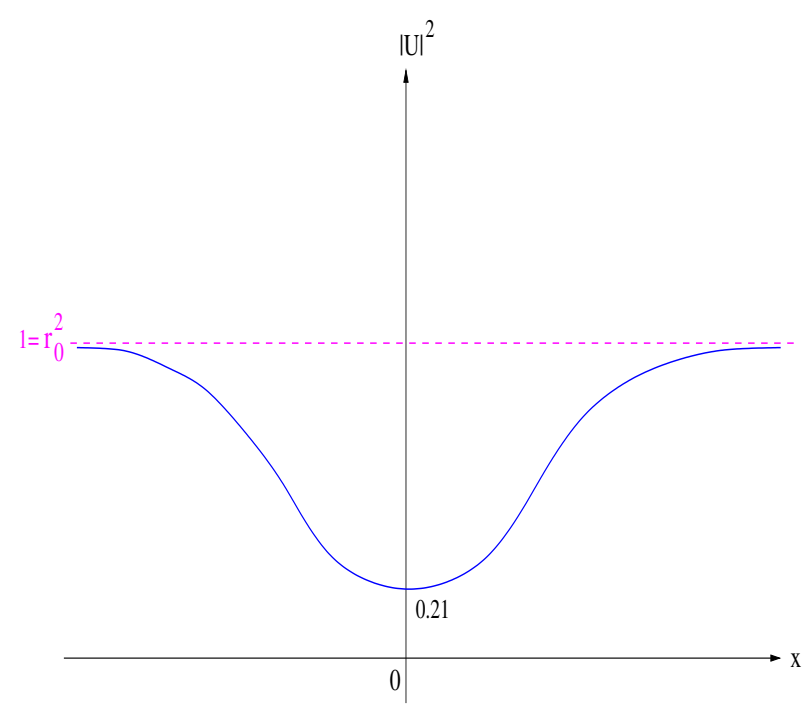

(b)

Figure 27: Graphs of (a) $\mathcal{V}_{c}$ and (b) $|U|^{2}$ for $c=\sqrt{1-0.6^{2}}=0.8<\mathfrak{c}_{s}=1$ 


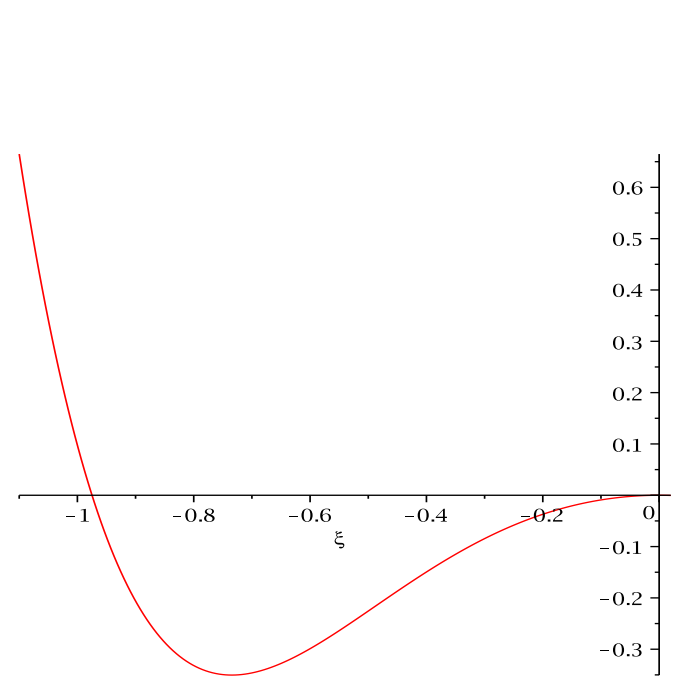

(a)

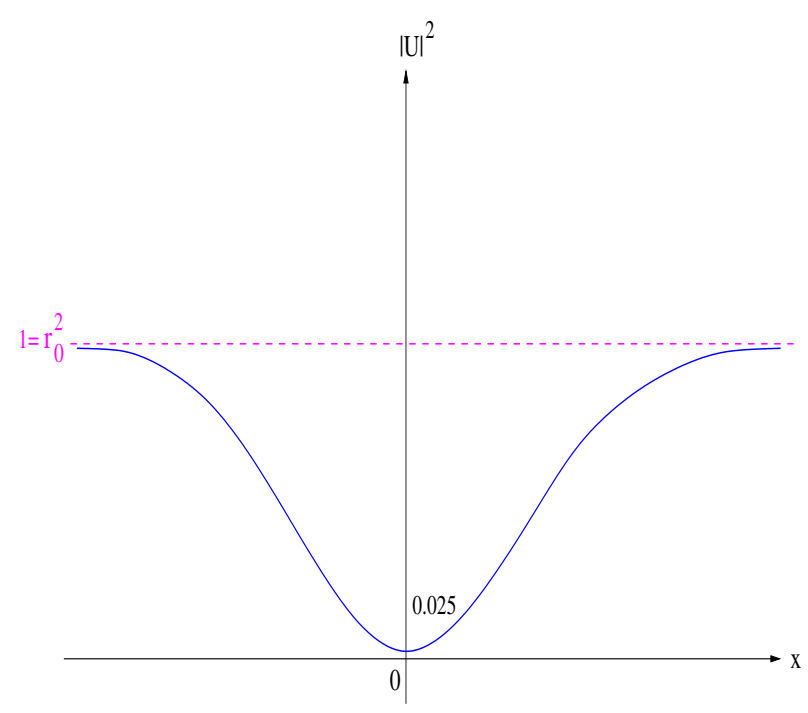

(b)

Figure 28: Graphs of (a) $\mathcal{V}_{c}$ and (b) $|U|^{2}$ for $c=\sqrt{1-0.95^{2}} \simeq 0.3122<\mathfrak{c}_{s}=1$

The graphs of energy and momentum of the solutions with respect to the speed, and the $(E, P)$ diagram (the straight line is $E=\mathfrak{c}_{s} P$ ) are thus

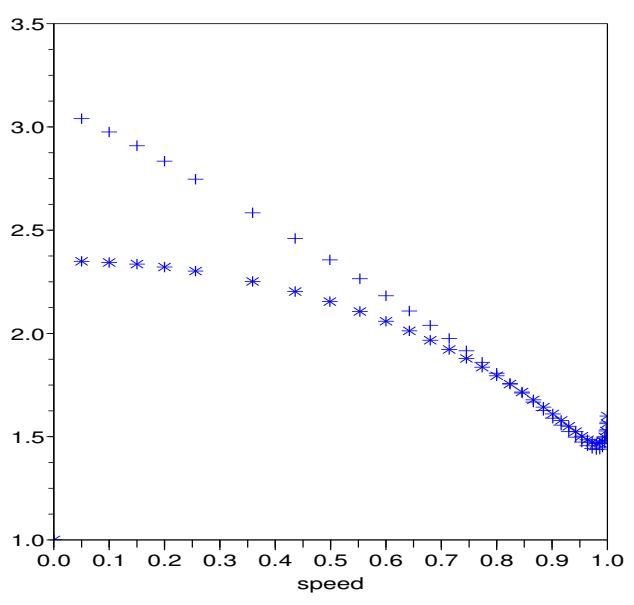

(a)

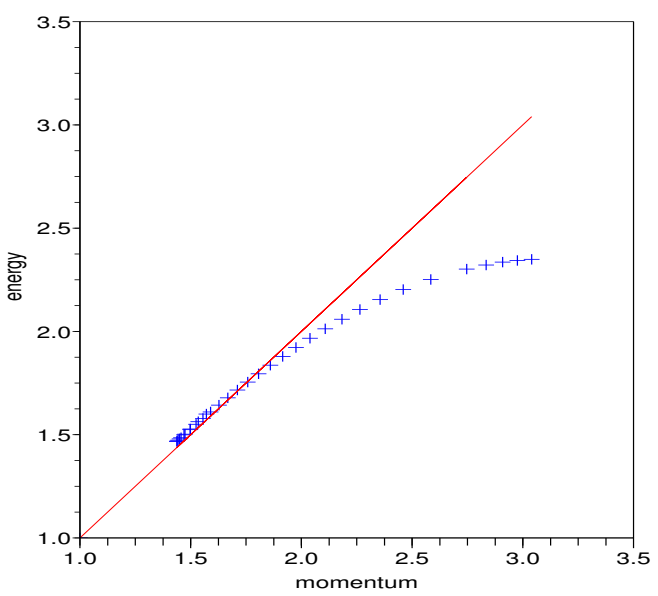

(b)

Figure 29: (a) Energy $\left(^{*}\right)$ and momentum $(+)$ vs. speed; (b) $(E, P)$ diagram

The diagrams are therefore of the following type. 


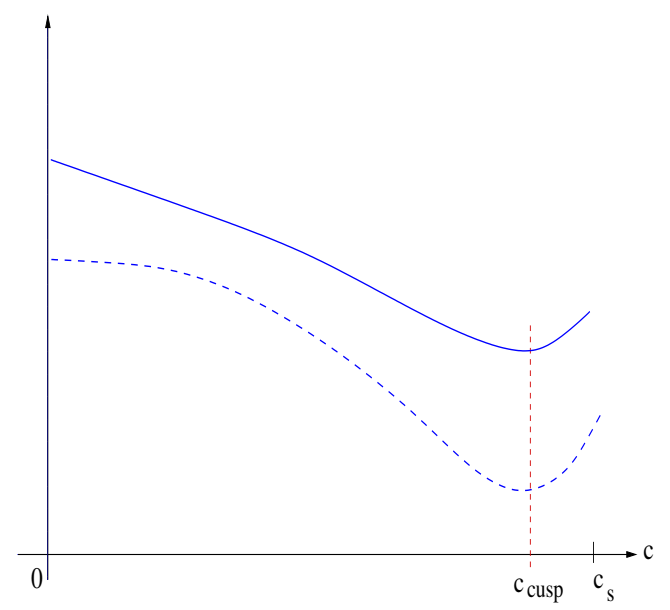

(a)

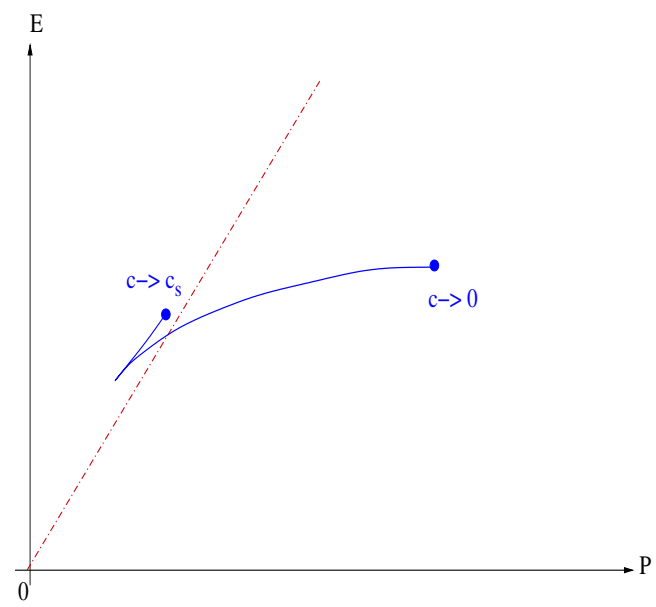

(b)

Figure 30: (a) Energy (dashed curve) and momentum (full curve) vs. speed; (b) $(E, P)$ diagram

Comments. In the $(E, P)$ diagram above, we see that there do not exist traveling waves with small energy or momentum. In the transonic limit, we have convergence to a sonic nontrivial traveling wave $U_{\mathfrak{c}_{s}}$. Notice that from the expressions of the energy and the momentum in subsection 1.2, it follows that for this sonic wave,

$$
E\left(U_{\mathfrak{c}_{s}}\right)-\mathfrak{c}_{s} P\left(U_{\mathfrak{c}_{s}}\right)=\operatorname{sgn}\left(\xi_{\mathfrak{c}_{s}}\right) \int_{0}^{\xi_{\mathfrak{c}_{s}}} \frac{\sqrt{-\mathcal{V}(\xi)}}{r_{0}^{2}+\xi} d \xi>0
$$

since $\xi_{\mathfrak{c}_{s}} \neq 0$. Moreover, this gives another example where we observe a cusp.

\subsection{Example 4: a degenerate case}

We investigate now the case

$$
f(\varrho) \equiv-2(\varrho-1)+3(\varrho-1)^{2}-4(\varrho-1)^{3}+5(\varrho-1)^{4}-6(\varrho-1)^{5},
$$

for which

$$
V(\varrho)=(\varrho-1)^{2}-(\varrho-1)^{3}+(\varrho-1)^{4}-(\varrho-1)^{5}+(\varrho-1)^{6} \quad \text { and } \quad \mathcal{V}(\xi)=-4 \xi^{7},
$$

thus $r_{0}=1, \mathfrak{c}_{s}^{2}=4, \Gamma=\Gamma^{\prime}=0$ and the graphs of $f, V$ and $\mathcal{V}$ are

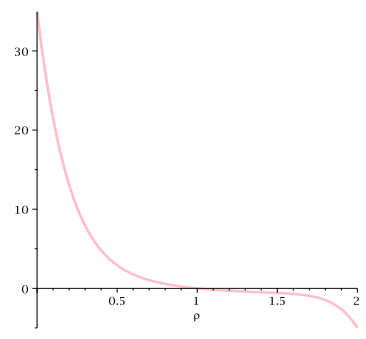

(a)

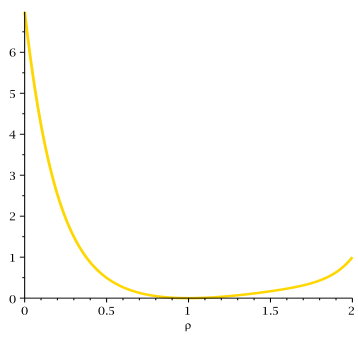

(b)

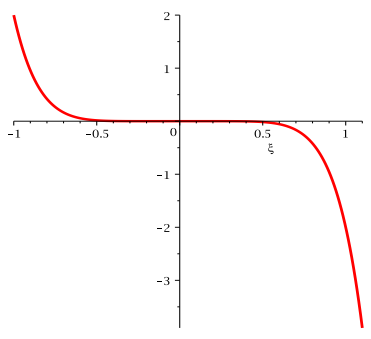

(c)

Figure 31: Graphs of (a) $f$, (b) $V$ and (c) $\mathcal{V}$

Let us point out that the function $\mathcal{V}$ is very flat at the origin, namely $\mathcal{V}(\xi)=-4 \xi^{7}$. As we have seen, the behaviour of $\mathcal{V}$ at the origin is related to the coefficients $\Gamma, \Gamma^{\prime}, \ldots$. The nonlinearity we consider 
here corresponds to the very degenerate situation where $\Gamma=\Gamma^{\prime}=\Gamma^{\prime \prime}=\ldots=\Gamma^{(6)}=0$ but $\Gamma^{(7)}<0$, so that the transonic limit is governed by the first supercritical ( $\mathrm{gKdV}$ ) equation with nonlinearity $\Gamma^{(6)} A^{5} \partial_{z} A$. This choice was motivated by the fact that it is the first integer for which we see a supercritical (gKdV) equation in the transonic limit, with energy and momentum diverging to $+\infty$. The graphs of the potential functions $\mathcal{V}_{c}$ will be qualitatively as for the Gross-Pitaevskii nonlinearity (figure 2) and thus we omit them. The graphs of energy and momentum of the solutions with respect to the speed, and the $(E, P)$ diagram (the straight line is $E=\mathfrak{c}_{s} P$ ) are given below. Let us mention that for the numerical integration of the energy and momentum, since $\mathcal{V}(\xi)=-4 \xi^{7}$ is very flat near $\xi=0$, we use the fact that the nontrivial zero $\xi$ of the polynomial $\mathcal{V}(\xi)-\varepsilon^{2} \xi^{2}=-4 \xi^{7}-\varepsilon^{2} \xi^{2}$ is simply $\xi_{\varepsilon}=-\left(\varepsilon^{2} / 4\right)^{\frac{1}{5}}$, and use the change of variable $\xi=\xi_{\varepsilon} t$ and simplify the expression before performing the numerical integration.

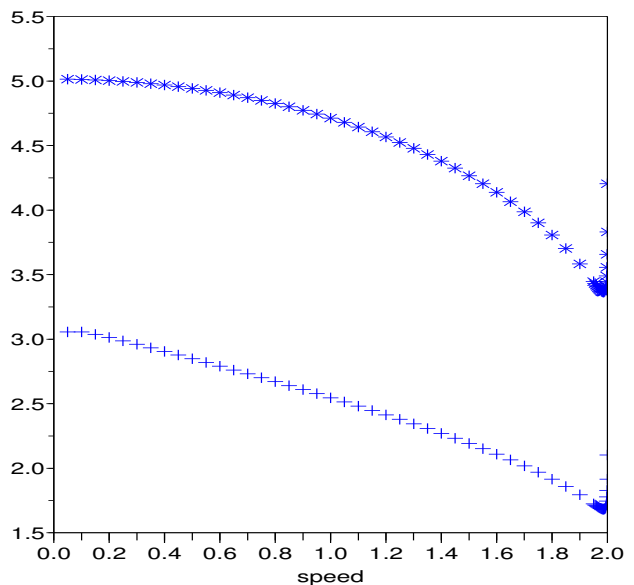

(a)

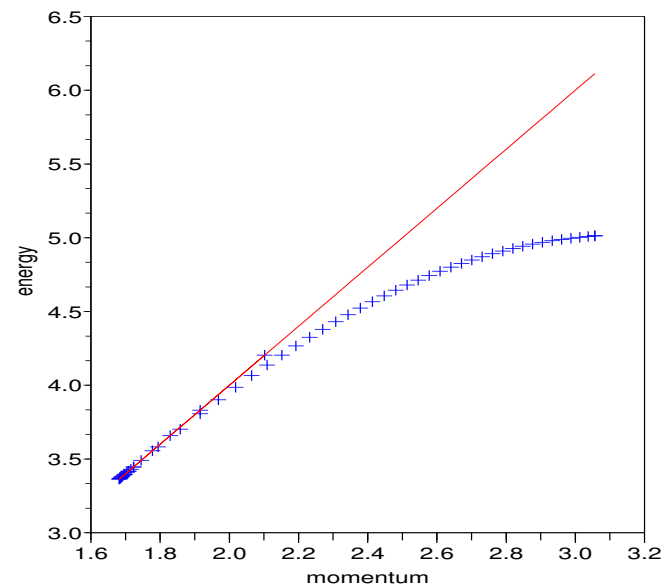

(b)

Figure 32: (a) Energy $\left(^{*}\right)$ and momentum (+) vs. speed; (b) $(E, P)$ diagram

Qualitatively, the diagrams have therefore the following behaviour.

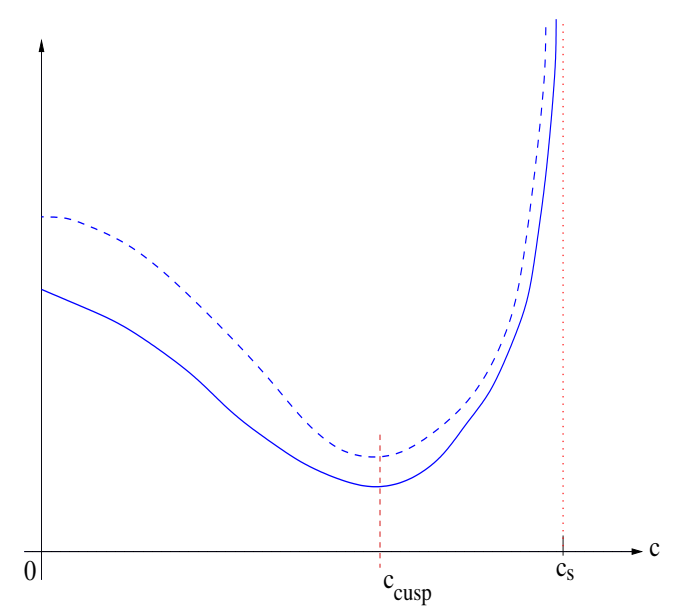

(a)

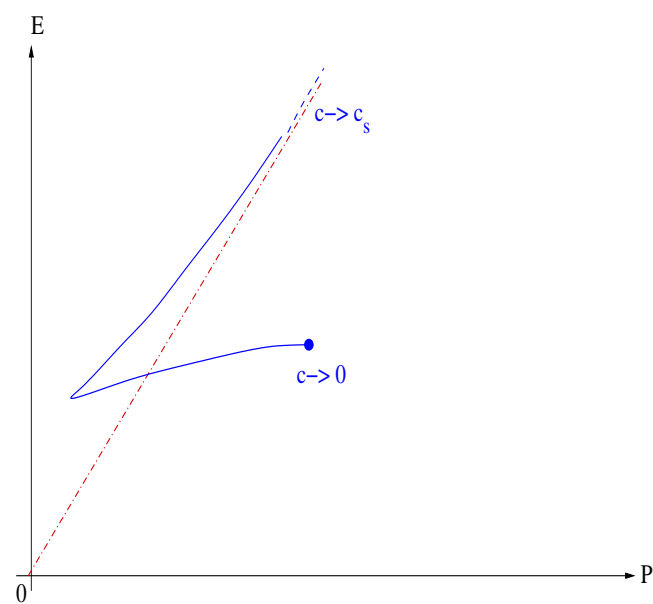

(b)

Figure 33: (a) Energy (dashed curve) and momentum (full curve) vs. speed; (b) (E, $P$ ) diagram

Comments. As in example 3, there do not exist traveling waves with small energy or momentum and we 
have a cusp in the $(E, P)$ diagram. Moreover, the transonic limit provides a branch with diverging energy and momentum (see the end of section 2.3). The divergence is not easy to see numerically since it is of order $\varepsilon^{-\frac{1}{5}}=\left(\mathfrak{c}_{s}^{2}-c^{2}\right)^{-\frac{1}{10}} \simeq\left(\mathfrak{c}_{s}-c\right)^{-\frac{1}{10}}$. However, we have seen that the straight line $E=\mathfrak{c}_{s} P$ is an asymptote.

\subsection{Example 5: a saturated nonlinearity}

In this example, we take, for some $\varrho_{0}>0$,

$$
f(\varrho) \equiv \exp \left(\frac{1-\varrho}{\varrho_{0}}\right)-1
$$

This type of nonlinearity saturates when $\varrho$ is large and can be found, for instance, in [20]. For this $f$, we have

$$
V(\varrho)=\varrho_{0}\left\{\exp \left(\frac{1-\varrho}{\varrho_{0}}\right)-1-\frac{1-\varrho}{\varrho_{0}}\right\} \quad \text { and } \quad \mathcal{V}(\xi)=\frac{2}{\varrho_{0}} \xi^{2}-4 \varrho_{0}(1+\xi)\left\{\exp \left(-\frac{\xi}{\varrho_{0}}\right)-1+\frac{\xi}{\varrho_{0}}\right\},
$$

thus $r_{0}=1, \mathfrak{c}_{s}^{2}=2 / \varrho_{0}, \Gamma=6-\frac{2}{\varrho_{0}}$. Therefore, the coefficient $\Gamma$ changes sign for $\varrho_{0}=1 / 3$. It should be noticed that $V(\varrho)$ grows just linearly at infinity, and that, for large $\xi, \mathcal{V}(\xi)$ tends to $+\infty$ quadratically if $\varrho_{0}>1 / 2$ and to $-\infty$ when $\varrho_{0} \leq 1 / 2$ (quadratically if $\varrho_{0}<1 / 2$ and linearly for $\varrho_{0}=1 / 2$ ). For $\varrho_{0}>0$, the graphs of $f$ and $V$ are typically

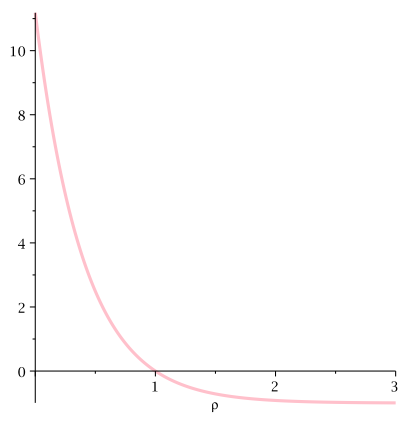

(a)

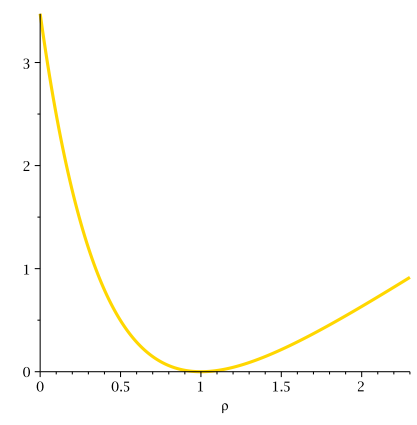

(b)

Figure 34: Graphs of (a) $f$ and (b) $V$

The graph of $\mathcal{V}$ depends on the sign of $\Gamma$ and whether $\varrho_{0}$ is less or larger than $1 / 2$.

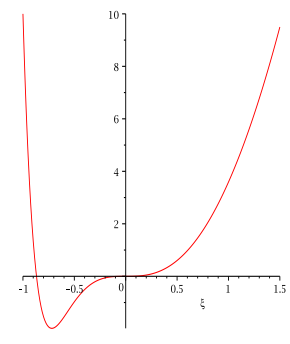

(a)

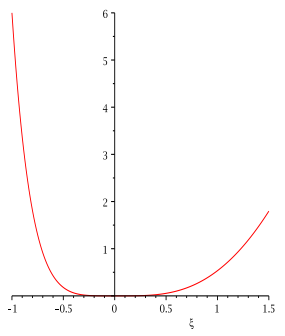

(b)

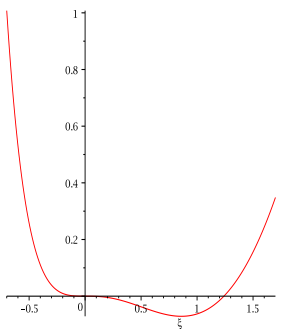

(c)

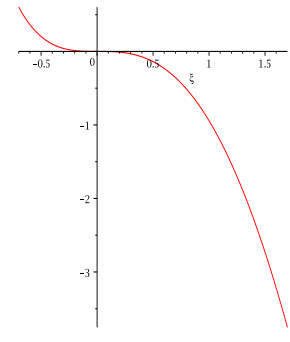

(d)

Figure 35: Graphs of $\mathcal{V}$ for (a) $\varrho_{0}=0.2$; (b) $\varrho_{0}=1 / 3$; (c) $\varrho_{0}=0.4$; (d) $\varrho_{0}=1$ 
We pursue the study in the case $\varrho_{0}=0.4$, thus $\mathfrak{c}_{s}^{2}=5, \Gamma=1$. Notice that we have then a sonic traveling wave solution as in example 3 . In order to see what happens, we draw the potentials $\mathcal{V}_{c}$ by hand: the scales are not respected, but the abscissae we indicate are correct. We begin with the solution in the domain $\xi<0$, where we have a situation similar to the Gross-Pitaevskii case.

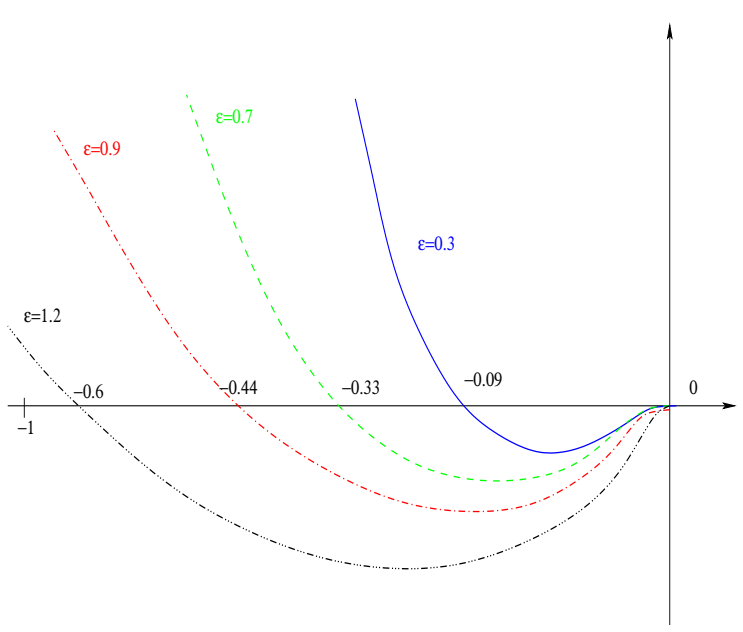

(a)

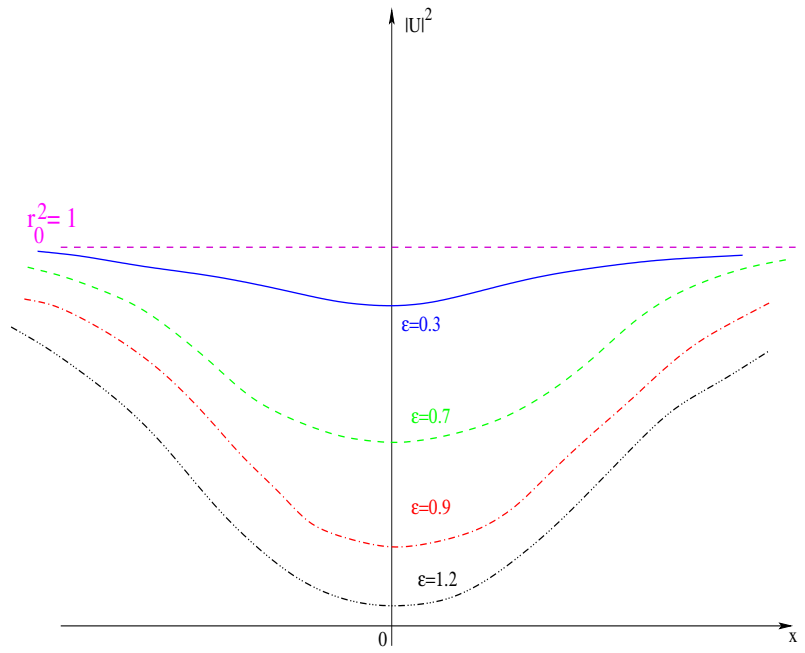

(b)

Figure 36: Region $\xi<0$ : (a) graphs of $\mathcal{V}_{c}$; (b) corresponding $|U|^{2}$ (scales are not respected)

We then turn to the part where $\xi>0$, for which we have a traveling wave with speed $c=\mathfrak{c}_{s}$.

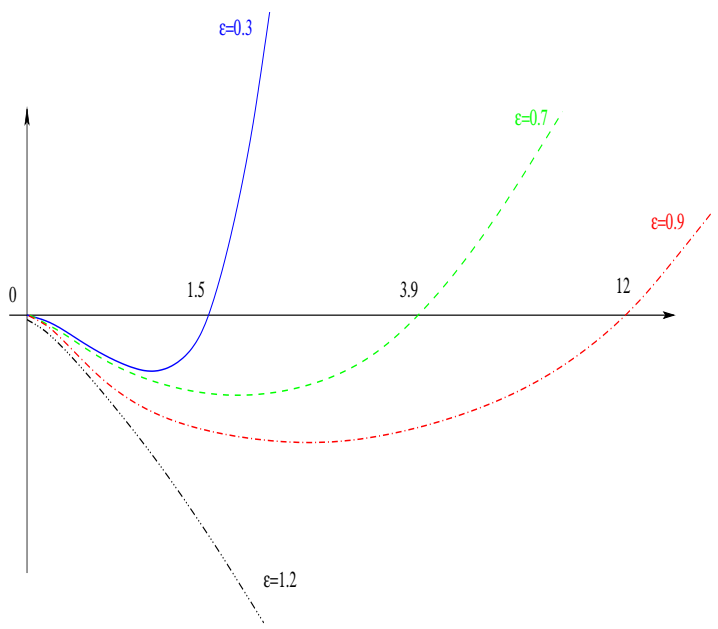

(a)

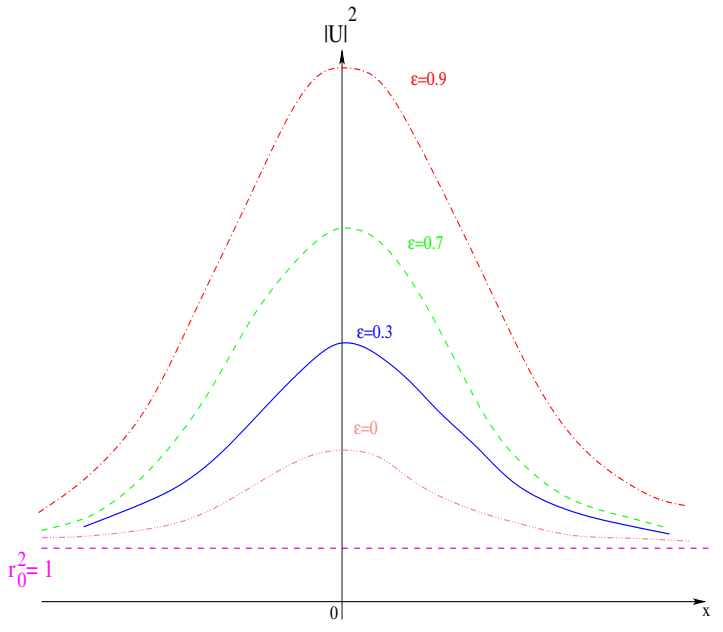

(b)

Figure 37: region $\xi>0$ : (a) graphs of $\mathcal{V}_{c}$; (b) corresponding $|U|^{2}$ (scales are not respected)

Notice that $\frac{2}{\varrho_{0}}-4=1$ and for $\varepsilon \geq 1$, there no longer exist nontrivial traveling waves. This can be seen from the fact that, for $\varepsilon \neq 1$, we have $\mathcal{V}_{c}(\xi) \sim\left(\frac{2}{\varrho_{0}}-4-\varepsilon^{2}\right) \xi^{2}=\left(1-\varepsilon^{2}\right) \xi^{2}$ as $\xi \rightarrow+\infty$, and the coefficient $1-\varepsilon^{2}$ changes sign at $\varepsilon=1$. Actually, for $0 \leq \varepsilon<1$, the potential $\mathcal{V}_{c}$ is negative in $\left(0, \xi_{\varepsilon}\right)$ and positive in $\left(\xi_{\varepsilon},+\infty\right)$, for some positive number $\xi_{\varepsilon}$ such that $\xi_{\varepsilon} \simeq \frac{1.8}{1-\varepsilon}$ as $\varepsilon \rightarrow 1^{-}$(or equivalently $c \rightarrow 2^{+}$). In figure 37 , it then follows that the maximum value of $|U|^{2}$, namely $r_{0}^{2}+\xi_{\varepsilon}=1+\xi_{\varepsilon}$, diverges like $\frac{1.8}{1-\varepsilon}$ as $\varepsilon \rightarrow 1^{-}$. For $\varepsilon \geq 1, \mathcal{V}_{c}$ is negative in $(0,+\infty)$. The diagrams we obtain are as follows. Notice that in (b), the divergence is rather strong and hence easily seen numerically (we are actually able to compute much larger values of $E$ and $P$ ). 


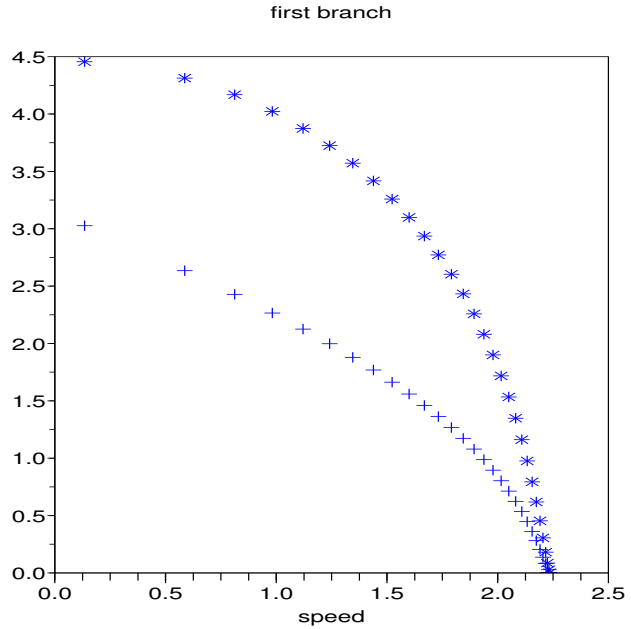

(a)

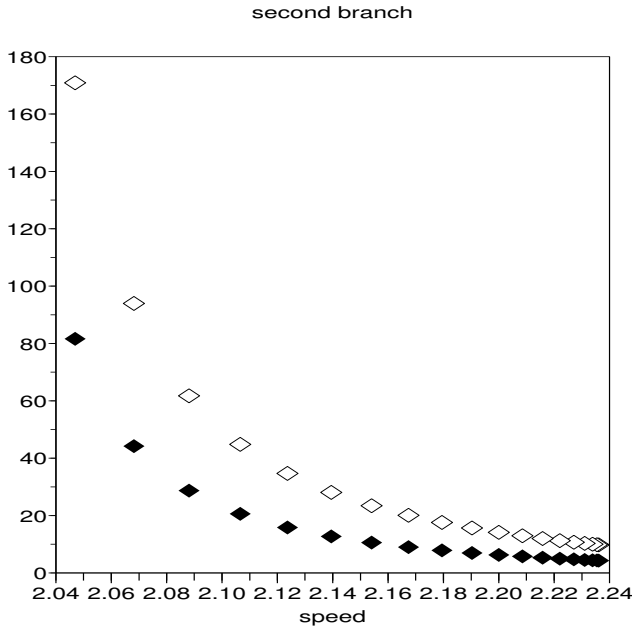

(b)

Figure 38: (a) Energy $(*)$ and momentum $(+)$ vs. speed for the first branch; (b) energy $(\diamond)$ and momentum $(\checkmark)$ vs. speed for the second branch

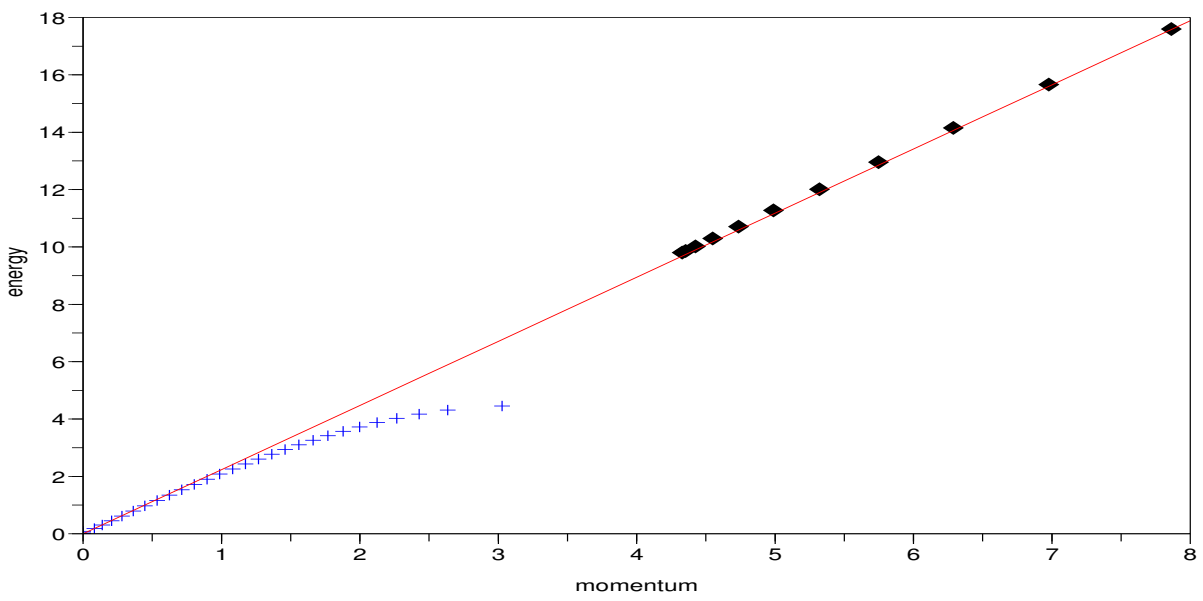

Figure 39: $(E, P)$ diagram for the first branch $(+)$ and the second branch $(\downarrow)$

The global behaviour of the diagrams is then: 


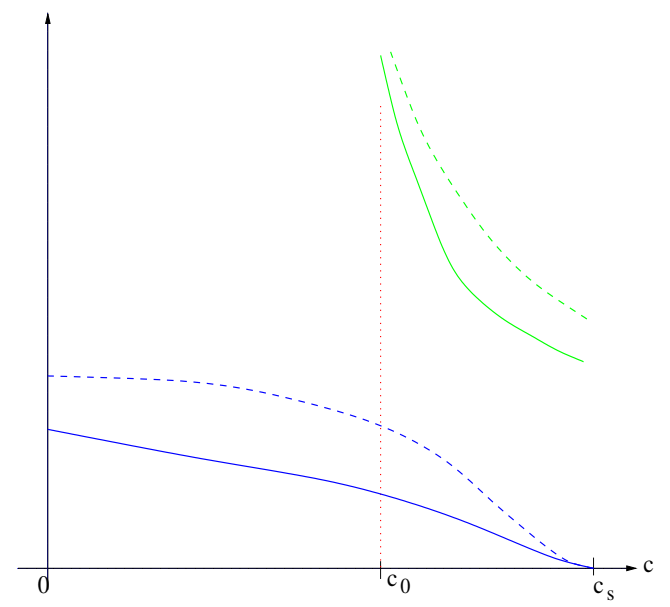

(a)

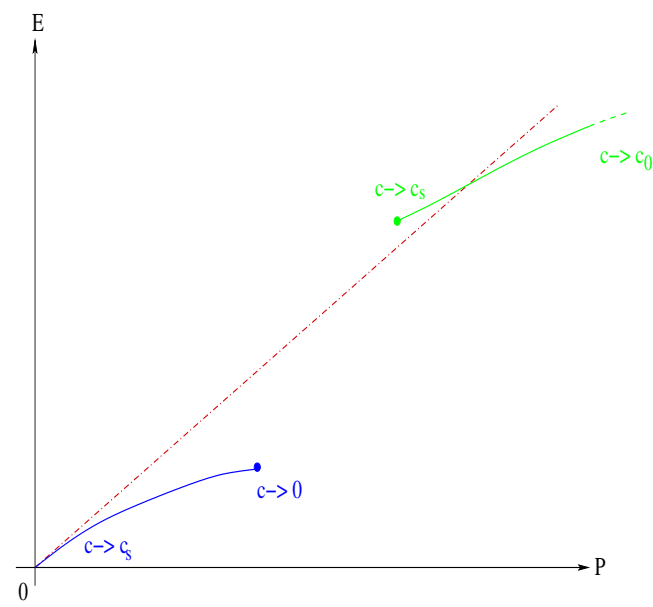

(b)

Figure 40: (a) Energy (dashed curve) and momentum (full curve) vs. speed; (b) $(E, P)$ diagram

Comments. We point out that two facts are combined in this example. The first one is that for $\varrho_{0}=$ $0.4 \in(1 / 3,1 / 2)$, we have two solutions for speeds $c$ close to $\mathfrak{c}_{s}$ (this is also the case when $\varrho_{0}=1 / 3$, as in example 1): one with small energy, and the other one with an energy of order one. On the other hand, due to the saturation effect, the traveling wave solutions are not uniformly bounded: we obtain a branch of solutions that blows up in $L^{\infty}(\mathbb{R})$ like in figure 37. This phenomenon also holds for $\varrho_{0}<1 / 2$ but not for the second nonlinearity in (1). These two effects can of course be encontered separately. We also would like to point out that we have nontrivial traveling waves only for $0<P \leq \pi \simeq 3.14$ and $P \geq 4.33$, but not for $3.14 \leq P \leq 4.33$.

\subsection{Example 6: the cubic-quintic nonlinearity}

We consider finally the cubic-quintic nonlinearity

$$
f(\varrho) \equiv-(\varrho-1)-3(\varrho-1)^{2},
$$

for which

$$
V(\varrho)=\frac{1}{2}(\varrho-1)^{2}+(\varrho-1)^{3} \quad \text { and } \quad \mathcal{V}(\xi)=-6 \xi^{3}-4 \xi^{4},
$$

thus $r_{0}=1, \mathfrak{c}_{s}^{2}=2, \Gamma=24$ and the graphs of $f, V$ and $\mathcal{V}$ are

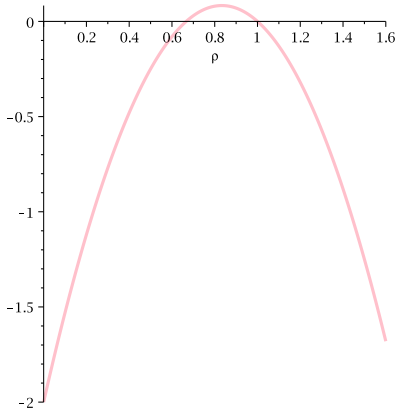

(a)

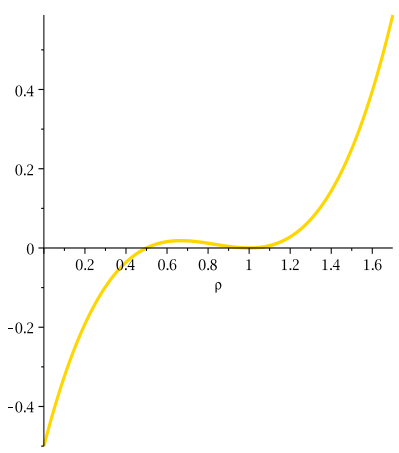

(b)

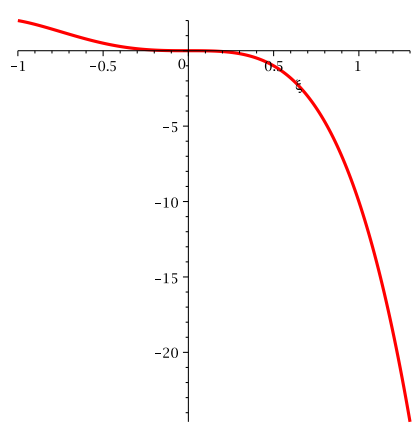

(c)

Figure 41: Graphs of (a) $f$, (b) $V$ and (c) $\mathcal{V}$ 
This nonlinearity was extensively studied in the physical literature. We just recall the study in [5] (see also other papers by I. Barashenkov and co-authors). An important feature is that $f$ is increasing near $\varrho=0$ and that the potential $V$ takes negative values near the origin. The energy and momentum with respect to the speed $c$ and the $(E, P)$ diagram for this case (see [5]) are given below.

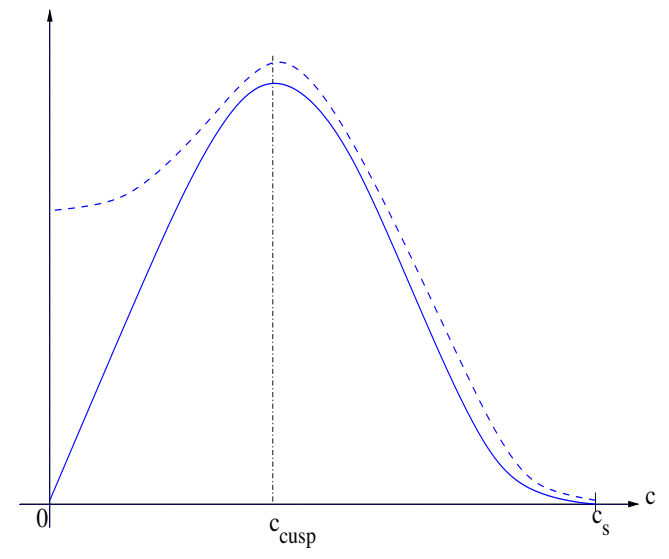

(a)

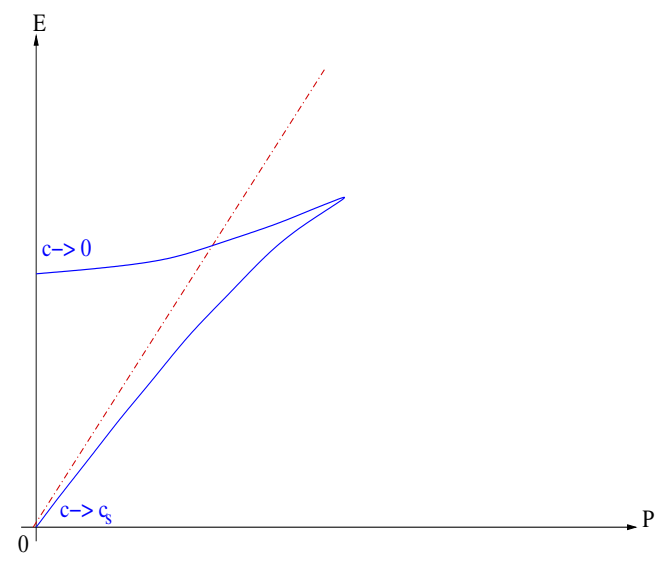

(b)

Figure 42: (a) Energy (dashed curve) and momentum (full curve), (b) (E,P) diagram

Comments. This nonlinearity provides an example of a cusp where $E$ and $P$ both reach a local maximum. As $c \rightarrow 0$, the traveling wave $U_{c}$ is clearly such that $|U|^{2}$ takes some values in the region where $V<0$. This time, the stationnary solution $U_{0}$ is a bubble and not a kink as in the other examples. In example 2, there is some speed $c=c_{0} \in\left(0, \mathfrak{c}_{s}\right)$ which is missing in the spectrum of speeds. It is possible to make $c_{0}=0$ by taking a degenerate situation of the cubic-quintic nonlinearity for which $\varrho=0$ is a zero of $V$, that is

$$
f(\varrho)=-2(\varrho-1)-3(\varrho-1)^{2}, \quad V(\varrho)=\varrho(\varrho-1)^{2}, \quad \mathcal{V}_{c}(\xi)=\xi^{2}\left[c^{2}-4(1+\xi)^{2}\right] .
$$

\subsection{Conclusions}

We have studied the qualitative properties of the traveling waves of the Nonlinear Schrödinger equation with nonzero condition at infinity for a general nonlinearity. If the energy-momentum diagram is well-known for the Gross-Pitaevskii equation, we have shown that the qualitative properties of the traveling waves solutions can not be easily deduced from the global shape of the nonlinearity. In particular, through various model cases for which the nonlinearity is smooth and decreasing (as is the Gross-Pitaevkii one), we have put forward a great variety of behaviours: multiplicity of solutions; branches with diverging energy and momentum; nonexistence of traveling wave for some $c_{0} \in\left(0, \mathfrak{c}_{s}\right)$; branches in the $(E, P)$ diagram that cross; existence of sonic traveling wave; transonic limit governed by the $(\mathrm{mKdV})$ equation, or more generally by the $(\mathrm{gKdV})$ solitary wave equation instead of the usual (KdV) one; existence of cusps... In [10], we perform numerical simulations in dimension two for the model cases we have studied here.

Acknowledgements: The support of the ANR ArDyPitEq is greatfully acknowledged. I would like to thank M. Mariş for helpfull comments about this work, as well as the referees for suggestions that improved the presentation.

\section{References}

[1] M. Abid, C. Huepe, S. Metens, C. Nore, C. T. Pham, L. S. Tuckerman and M. E. Brachet, Gross-Pitaevskii dynamics of Bose-Einstein condensates and superfluid turbulence. Fluid Dynamics Research, 33, 5-6 (2003), 509-544. 
[2] V. ARnol'D, Ordinary differential equations. Springer textbook [translation of the russian (1984)] (1992).

[3] I. Barashenkov, Stability Criterion for Dark Solitons. Phys. Rev. Lett. 77, 1193-1197 (1996).

[4] I. Barashenkov and V. Makhankov, Soliton-like bubbles in a system of interacting Bosons. Phys. Lett. A 128, 52-56 (1988).

[5] I. Barashenkov and E. Panova, Stability and evolution of the quiescent and travelling solitonic bubbles Physica D: Nonlinear Phenomena 69, 1-2 (1993), 114-134.

[6] F. Béthuel, P. Gravejat and J-C. Saut, Existence and properties of travelling waves for the Gross-Pitaevskii equation. Stationary and time dependent Gross-Pitaevskii equations, 55-103, Contemp. Math., 473, Amer. Math. Soc., Providence, RI, (2008).

[7] F. Béthuel, P. Gravejat, J-C. Saut and D. Smets, Orbital stability of the black soliton to the Gross-Pitaevskii equation, Indiana Math. Univ. J. 57, 6, (2008) 2611-2642.

[8] M. Bogdan, A. Kovalev and A. Kosevich, Stability criterion in imperfect Bose gas Fiz. Nizk. Temp. 15 (1989) 511-513 [in Russian].

[9] J. Bona, P. Souganidis and W. Strauss, Stability and instability of solitary waves of Korteweg-de Vries type. Proc. R. Soc. Lond. A, 411 (1987), 395-412.

[10] D. Chiron And C. Scheid, Traveling waves for the Nonlinear Schrödinger Equation with general nonlinearity in dimension two. Work in progress.

[11] A. De Bouard, Instability of stationary bubbles. SIAM J. Math. Anal. 26, no. 3 (1995), 566-582.

[12] L. Di Menza and C. Gallo, The black solitons of one-dimensional NLS equations. Nonlinearity 20, no. 2 (2007), 461-496.

[13] C. Gallo, The Cauchy problem for defocusing nonlinear Schrödinger equations with non-vanishing initial data at infinity. Comm. Partial Differential Equations 33, no. 4-6 (2008), 729-771.

[14] P. GÉRARD, The Gross-Pitaevskii equation in the energy space. in Stationary and time dependent Gross-Pitaevskii equations, 129-148, Contemp. Math., 473, Amer. Math. Soc., Providence, RI, (2008).

[15] P. GÉrard And Z. Zhang, Orbital stability of traveling waves for the one-dimensional Gross-Pitaevskii equation. J. Math. Pures Appl. (9) 91, no. 2 (2009), 178-210.

[16] V. Ginzburg and L. Pitaevskit, On the theory of superfluidity. Sov. Phys. JETP 34 (1958), 1240.

[17] M. Grillakis, J. Shatah and W. Strauss, Stability theory of solitary waves in the presence of symmetry I, J. Funct. Anal. 74 (1987), 160-197.

[18] E. Gross, Hydrodynamics of a superfluid condensate, J. Math. Phys. 4 (2) (1963), 195-20\%.

[19] C. Jones and P. Roberts, Motion in a Bose condensate IV. Axisymmetric solitary waves. J. Phys. A: Math. Gen., 15 (1982), 2599-2619.

[20] Y. Kivshar, D. Anderson and M. Lisak, Modulational instabilities and dark solitons in a generalized nonlinear Schrödinger equation. Phys. Scr. 47 (1993), 679-681.

[21] Y. Kivshar and W. Krolikowski, Instabilities of dark solitons. Optics Letters, 20, 14 (1995), $1527-1529$.

[22] Y. S. Kivshar and B. Luther-Davies, Dark optical solitons: physics and applications. Physics Reports 298 (1998), 81-197.

[23] E. B. Kolomeisky, T. J. Newman, J. P. Straley and X. Qi, Low-Dimensional Bose Liquids: Beyond the Gross-Pitaevskii Approximation. Phys. Rev. Lett. 85 (2000), 1146-1149. 
[24] Z. Lin, Stability and instability of traveling solitonic bubbles. Adv. Differential Equations 7, no. 8 (2002), 897-918.

[25] M. MARIş, Nonexistence of supersonic traveling waves for nonlinear Schrödinger equations with nonzero conditions at infinity. SIAM J. Math. Anal. 40, no. 3 (2008), 1076-1103.

[26] Y. Martel and F. Merle, Instability of solitons for the critical generalized Korteweg-de Vries equation. Geom. Funct. Anal. 11, no. 1 (2001), 74-123.

[27] Y. Martel And F. Merle, Blow up in finite time and dynamics of blow up solutions for the $L^{2}$-critical generalized KdV equation. J. Amer. Math. Soc. 15, no. 3 (2002), 617-664.

[28] P. Roberts And N. Berloff, Nonlinear Schrödinger equation as a model of superfluid helium. In "Quantized Vortex Dynamics and Superfluid Turbulence" edited by C.F. Barenghi, R.J. Donnelly and W.F. Vinen, Lecture Notes in Physics, volume 571, Springer-Verlag, 2001.

[29] P. Souganidis and W. Strauss, Instability of a class of dispersive solitary waves. Proc. Roy. Soc. Edinburgh A, 114 (1990), 195-212.

[30] T. Tsuzuki, Nonlinear waves in the Pitaevskii-Gross equation. J. Low Temp. Phys. 4, no. 4 (1971), $441-45 \%$

[31] V. Zakharov and A. Kuznetsov, Multi-scale expansion in the theory of systems integrable by the inverse scattering transform. Physica D, 18 (1-3) (1986), 455-463.

[32] P. Zhidkov, Korteweg-de-Vries and Nonlinear Schrödinger Equations: Qualitative Theory. Lecture Notes in Mathematics 1756, (2001) Springer-Verlag. 